\title{
Pedestrian Attribute Recognition: A Survey
}

\author{
Xiao Wang, Shaofei Zheng, Rui Yang, Bin Luo, Jin Tang
}

\begin{abstract}
Recognizing pedestrian attributes is an important task in computer vision community due to it plays an important role in video surveillance. Many algorithms has been proposed to handle this task. The goal of this paper is to review existing works using traditional methods or based on deep learning networks. Firstly, we introduce the background of pedestrian attributes recognition (PAR, for short), including the fundamental concepts of pedestrian attributes and corresponding challenges. Secondly, we introduce existing benchmarks, including popular datasets and evaluation criterion. Thirdly, we analyse the concept of multi-task learning and multi-label learning, and also explain the relations between these two learning algorithms and pedestrian attribute recognition. We also review some popular network architectures which have widely applied in the deep learning community. Fourthly, we analyse popular solutions for this task, such as attributes group, part-based, etc. Fifthly, we shown some applications which takes pedestrian attributes into consideration and achieve better performance. Finally, we summarized this paper and give several possible research directions for pedestrian attributes recognition. The project page of this paper can be found from the following website: https://sites.google.com/view/ahu-pedestrianattributes/.
\end{abstract}

Index Terms-Pedestrian Attributes Recognition, Multi-label Learning, Deep Learning, Convolutional Neural Network, Recurrent Neural Network, Graph Convolutional Network, Visual Attention

\section{INTRODUCTION}

$\mathrm{P}$ EDESTRIAN attributes, are humanly searchable semantic descriptions and can be used as soft-biometrics in visual surveillance, with applications in person re-identification, face verification, and human identification. Pedestrian attributes recognition (PAR) aims at mining the attributes of target people when given person image, as shown in Figure 1. Different from low-level features, such as HOG, LBP or deep features, attributes can be seen as high-level semantic information which is more robust to viewpoint changes and viewing condition diversity. Hence, many tasks in computer vision integrate the attribute information into their algorithms to achieve better performance, such as person reID, person detection. Although many works has been proposed on this topic, however, pedestrian attributes recognition is still an unsolved problem due to challenging factors, such as view point change, low illumination, low resolution and so on.

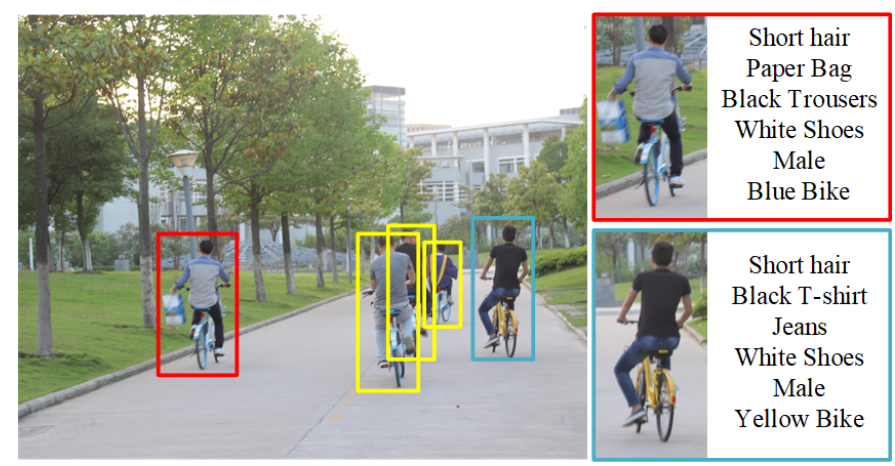

Figure 1. Pedestrian attributes recognition is a key element in video surveillance. Given a person image, pedestrian attributes recognition aims at predict a group of attributes to describe the characteristic of this person from a pre-defined attribute list, for example, the attributes of man in red bounding box are: short hair, with paper bag, black trousers, etc.

* The authors are all from School of Computer Science and Technology, Anhui University, Hefei, China. Email: \{wangxiaocvpr, shaofeizheng, yangruiahu\}@foxmail.com, \{luobin, t $j\} @ a h u . e d u . c n$.
Traditional pedestrian attributes recognition methods usually focus on developing robust feature representation from the perspectives of hand-crafted features, powerful classifiers or attributes relations. Some milestones including HOG [1], SIFT [2], SVM [3] or CRF model [4]. However, the reports on large-scale benchmark evaluations suggest that the performance of these traditional algorithms is far from the requirement of realistic applications.

Over the past several years, deep learning have achieved an impressive performance due to their success on automatic feature extraction using multi-layer nonlinear transformation, especially in computer vision, speech recognition and natural language processing. Several deep learning based attribute recognition algorithms has been proposed based on these breakthroughs, such as [5] [6] [6] [7] [8] [9] [10] [11] [12] [13] [14] [15] [16] [17] [18] [19] [20] [21] [22] [23] [24] [25] [26], [27] [28] [29] [30] [31] [32] [33].

Although so many papers have been proposed, until now, there exists no work to make a detailed survey, comprehensive evaluation and insightful analysis on these attribute recognition algorithms. In this paper, we summarize existing works on pedestrian attributes recognition, including traditional methods and popular deep learning based algorithms, to better understand this direction and help other researchers to quickly capture main pipeline and latest research frontier. Specifically speaking, we attempt to address the following several important issues: (1) What is the connection and difference between traditional and deep-learningbased pedestrian attributes recognition algorithms? We analyse traditional and deep learning based algorithms from different classification rules, such as part-based, group-based or end-to-end learning; (2) How the pedestrian attributes help other related computer vision tasks? We also review some person attributes guided computer vision tasks, such as person re-identification, object detection, person tracking, to fully demonstrate the effectiveness and widely application in many other related tasks; (3) How to better leverage deep networks for pedestrian attributes recognition and what is the future direction of the development on attribute recognition? By evaluating existing person attributes recognition 


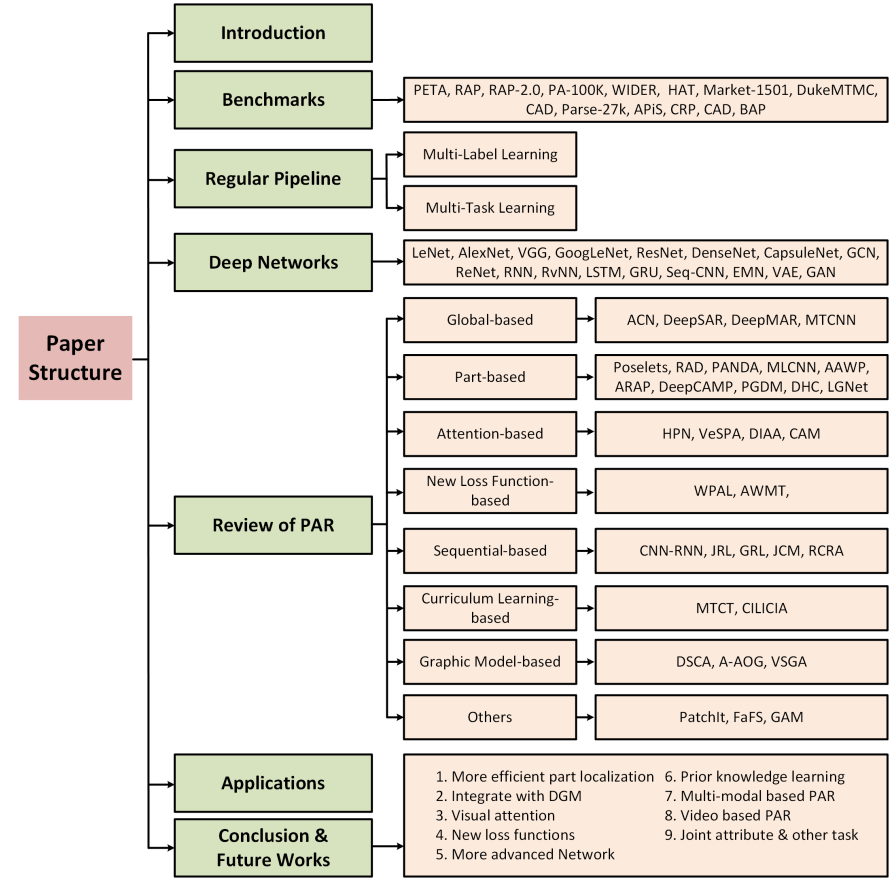

Figure 2. The structure of this survey on pedestrian attributes recognition.

algorithms and some top-ranked baseline methods, we make some useful conclusions and provide some possible research directions.

The remainder of this paper is organized as follows. In Section 2, we briefly introduce the problem formulation of pedestrian attributes recognition and some challenging factors. In Section 3 , we list some popular benchmarks for this task and report corresponding recognition performance of baseline methods. After that, we review existing methods in Section 4 and Section 6 from different categories. We also divide these methods into eight domains, including: global based, local parts based, visual attention based, sequential prediction based, new designed loss function based, curriculum learning based, graphic model based and others algorithms. In Section 7, we show some examples which can integrate attributes into consideration and achieve better performance. Finally, we summarize this paper and provide some possible research points for this direction in Section 8. To better visualize and understand the structure of this paper, we give a figure as shown in Figure 2.

\section{Problem Formulation and Challenges}

Given a person image $\mathcal{I}$, pedestrian attributes recogniton aims at predict a group of attributes $a_{i}$ to describe the charachteristic of this person from a pre-defined attribute list $\mathcal{A}=\left\{a_{1}, a_{2}, \ldots, a_{L}\right\}$, as shown in Figure 1. This task can be handled from different ways, such as multi-label classification, binary classification, and many algorithms and benchmarks have been proposed. However, this task is still challenging due to the large intra-class variations in attribute categories (appearance diversity and appearance ambiguity [34]). We list challenging factors which may obviously influence the final recognize performance as follows (see Figure 3):

Multi-views. The images taken from different angles by the camera leads to the viewpoint issues for many computer vision

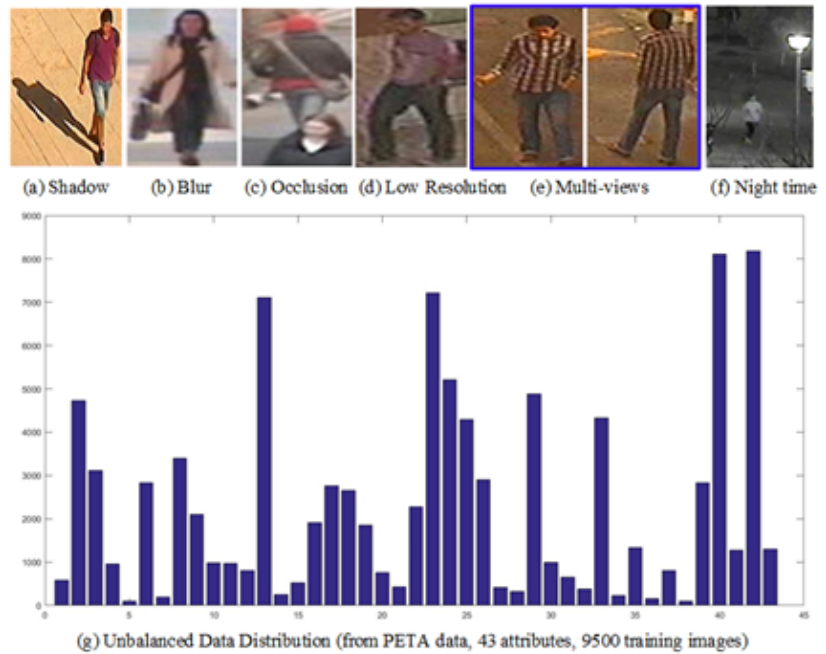

Figure 3. Some challenging factors in pedestrian attributes recognition.

tasks. Due to the body of human is not rigid, which further make the person attributes recognition more complicated.

Occlusion. Part of human body are occluded by other person or things will increase the difficulty of person attributes recognition. Because the pixel values introduced by the occluded parts may make the model confused and lead to wrong predictions.

Unbalanced Data Distribution. Each person have different attributes, hence, the number of attributes are variable which leads to unbalanced data distribution. As we all known, existing machine learning algorithms perform bad on these dataset.

Low Resolution. In practical scenarios, the resolution of images are rather low due to the high-quality cameras are rather expensive. Hence, the person attributes recognition need to be done under this environment.

Illumination. The images may taken from any time in 24 hours. Hence, the light condition may different in different time. The shadow maybe also taken in the person images and the images taken from night time maybe totally ineffective.

Blur. When person is moving, the images taken by the camera may blur. How to recognize person attributes correctly under this situation is a very challenging task.

\section{BENCHMARKS}

Unlike other tasks in computer vision, for pedestrian attribute recognition, the annotation of dataset contains many labels at different levels. For example, hair style and color, hat, glass , etc. are seen as specific low-level attributes and correspond to different areas of the images, while some attributes are abstract concepts, such as gender, orientation and age, which do not correspond to certain regions, we consider these attributes as high-level attributes. Furthermore, human attribute recognition are generally severely affected by environmental or contextual factors, such as viewpoints, occlusions and body parts, In order to facilitate the study, some datasets provider perspective, parts bounding box, occlusion, etc. attributes.

By reviewing related work in recent years, we have found and summarized several datasets which are used to research pedestrian attribute recognition, including PETA [34], RAP [35], RAP-2.0 [36], PA-100K [18], WIDER [16], Market-1501 [37] 
, DukeMTMC [37], Clothing Attributes Dataset [38], PARSE27K [5] [39], APiS [40], HAT [41], Berkeley-Attributes of People dataset [8] and CRP dataset [42]. Details of the attribute labels of these dataset can be found in our project page due to the limited space in this paper.

\subsection{Datasets}

PETA dataset [34] The PETA dataset is constructed from 10 publicly available small-scale datasets which used to researching person re-identification. This dataset consists of 19000 images, with resolution ranging from $17 \times 39$ to $169 \times 365$ pixels. Those 19000 images include 8705 persons, each annotated with 61 binary and 4 multi-class attributes, and it is randomly partitioned into 9,500 for training, 1,900 for verification and 7,600 for testing. One notable limitation is that the samples of one person in PETA dataset are only annotated once by randomly picking one exemplar image, and therefore share the same annotated attributes even though some of them might not be visible and some other attributes are ignored. Although this method is reasonable to a certain extent, it is not very suitable for visual perception.

PARSE27K [5], [39] The PARSE-27K datset derives from 8 video sequences of varying length taken by a moving camera in a city environment. Every 15th frame of the sequences was processed by the DPM pedestrian detector [48]. It contains 27,000 pedestrians and has a training (50\%), validation $(25 \%)$ and test (25\%) split. Each sample is manually annotated with 10 attribute labels which including 8 binary attributes such as is male?, has bag on left shoulder? and two orientation attributes with 4 and 8 discretizations. In PARSE-27K dataset, an attribute is called N/A label when it can not be decide because of occlusion, image boundaries, or any other reason.

RAP [35] The RAP dataset is collected from real indoor surveillance scenarios and 26 cameras are selected to acquire images, it contains 41585 samples with resolution ranging from $36 \times 92$ to $344 \times 554$, Specifically, there are 33268 images for training and the remains for testing. 72 fine-grained attributes (69 binary attribute and 3 multi-class attribute) are assigned to each image of this dataset. Three environmental and contextual factors, i.e., viewpoints, occlusion styles and body parts, are explicitly annotated. Six parts (spatial-temporal information, whole body attributes, accessories, postures and actions, occlusion, and parts attributes) are considered for attribute annotations.

RAP-2.0 [36] The RAP-2.0 dataset comes from a realistic HighDefinition $(1280 \times 720)$ surveillance network at an indoor shopping mall and all images are captured by 25 cameras scenes. This dataset contains 84928 images (2589 person identities) with resolution ranging from $33 \times 81$ to $415 \times 583$. Every image in this dataset has six types of labels, which are the same as RAP dataset and has 72 attribute labels. All samples were divided into three parts, of which 50957 for training, 16986 for validation and 16985 for testing.

HAT dataset [41] The HAT dataset originates in popular image sharing site Flickr. This dataset includes 9344 samples, of which 3500, 3500 and 2344 images are for training, validation and testing respectively. Every image in this dataset has 27 attributes and shows a considerable variation in pose (standing, sitting, running, turned back etc.), different ages (baby, teen, young, middle aged, elderly etc.), wearing different clothes (tee-shirt, suits, beachwear, shorts etc.) and accessories (sunglasses, bag etc.).
APiS dataset [40] The APiS dataset comes from four sources: KITTI [44] dataset, CBCL Street Scenes [45] (CBCLSS for short) dataset, INRIA [1] database and SVS dataset (Surveillance Video Sequences at a train station). A pedestrian detection approach [49] was performed to automatically locate candidate pedestrian regions, false positives and those too small images were deleted and finally, 3661 images obtained and each image larger than 90 pixels in height and 35 pixels in width. Each image is labeled with 11 binary attributes, such as "male", "long hair" and 2 multi value attributes, including upper body color and lower body color. The "ambiguous" indicates whether the corresponding attribute is uncertain or not. This dataset is separated into 5 equal sized subsets, the performance is evaluated with 5 -fold cross-validation and the 5 results from the 5 folds are further averaged to produce a single performance report.

Berkeley-Attributes of People Dataset, BAP [8] This dataset comes from the H3D [46] dataset and the PASCAL VOC 2010 [47] training and validation datasets for the person category, the low-resolution versions used in PASCAL are replaced by the full resolution equivalents on Flickr. All images was split into 2003 training, 2010 validation and 4022 test images by ensuring that no cropped images of different set come from the same source image and by maintaining a balanced distribution of the H3D and PASCAL images in each set. Each image was labeled with nine attributes. A label was considered as ground truth if at least 4 of the 5 annotators agreed on the value of the label. When an attribute is not determined to be present or absent, it is annotated as "unspecified".

PK-100K [18] The PA-100K dataset is constructed by images captured from 598 real outdoor surveillance cameras, it includes 100000 pedestrian images with resolution ranging from $50 \times 100$ to $758 \times 454$ and is to-date the largest dataset for pedestrian attribute recognition. The whole dataset is randomly split into training, validation and test sets with a ratio of $8: 1: 1$. Every image in this dataset was labelled by 26 attributes, and the label is either 0 or 1 , indicating the presence or absence of corresponding attributes respectively.

WIDER [16] The WIDER dataset comes from the 50574 WIDER images [43] that usually contain many people and huge human variations, a total of 13789 images were selected. Each image was annotated with a bounding box but no more than 20 people (with top resolutions) in a crowd image, resulting in 57524 boxes in total and 4+ boxes per image on average. Each person is labelled with 14 distinct attributes, resulting in a total of 805336 labels. This dataset was split into 5509 training, 1362 validation and 6918 test images.

Market1501-attribute [50] The Market-1501 dataset is collected by six cameras in front of a supermarket in Tsinghua University. There are 1501 identities and 32,668 annotated bounding boxes in this dataset. Each annotated identity is present in at least two cameras. This dataset was split into 751 training and 750 test identities, corresponding to 12936 and 19732 images respectively. The attributes are annotated in the identity level, every image in this dataset is annotated with 27 attributes. Note that the though there are 7 and 8 attributes for lower-body clothing and upperbody clothing, only one color is labelled as yes for an identity.

DukeMTMC-attribute [50] The images in DukeMTMCattribute dataset comes from Duke University. There are 1812 identities and 34183 annotated bounding boxes in the DukeMTMC-attribute dataset. This dataset contains 702 identities for training and 1110 identities for testing, corresponding to 16522 
Table 1

An overview of pedestrian attribute dataset (the \# denotes the number of).

\begin{tabular}{|c|c|c|c|c|}
\hline Dataset & \# Pedestrians & \#Attributes & Source & Project Page \\
\hline PETA [34] & 19000 & $\begin{array}{c}61 \text { binary and } 4 \\
\text { multi-class attributes }\end{array}$ & outdoor \& indoor & http://mmlab.ie.cuhk.edu.hk/projects/PETA.html \\
\hline RAP [35] & 41585 & $\begin{array}{c}69 \text { binary and } 3 \\
\text { multi-class attributes }\end{array}$ & indoor & http://rap.idealtest.org/ \\
\hline RAP-2.0 [36] & 84928 & $\begin{array}{c}69 \text { binary and } 3 \\
\text { multi-class attributes }\end{array}$ & indoor & https://drive.google.com/file/d/1hoPIB5NJKf3YGMvLFZnIYG5JDcZTxHph/view \\
\hline PA-100K [18] & 100000 & 26 binary attributes & outdoor & https://drive.google.com/drive/folders/0B5_Ra3JsEOyOUlhKM0VPZ1ZWR2M \\
\hline WIDER [16] & 13789 & 14 binary attributes & WIDER images [43] & http://mmlab.ie.cuhk.edu.hk/projects/WIDERAttribute.html \\
\hline Market-1501 [37] & 32668 & $\begin{array}{c}26 \text { binary and } 1 \\
\text { multi-class attributes }\end{array}$ & outdoor & https://github.com/vana77/Market-1501_Attribute \\
\hline DukeMTMC [37] & 34183 & 23 binary attributes & outdoor & https://github.com/vana77/DukeMTMC-attribute \\
\hline PARSE-27K [5] [39] & 27000 & $\begin{array}{c}\text { 8 binary and } 2 \\
\text { multi-class orientation attributes }\end{array}$ & outdoor & https://www.vision.rwth-aachen.de/page/parse27k \\
\hline APiS [40] & 3661 & $\begin{array}{c}11 \text { binary and } 2 \\
\text { multi-class attributes }\end{array}$ & $\begin{array}{c}\text { KITTI [44], } \\
\text { CBCL Street Scenes [45], } \\
\text { INRIA [1] and SVS }\end{array}$ & http://www.cbsr.ia.ac.cn/english/APiS-1.0-Database.html \\
\hline HAT [41] & 9344 & 27 binary attributes & image site Flickr & https://jurie.users.greyc.fr/datasets/hat.html \\
\hline CRP [42] & 27454 & $\begin{array}{l}1 \text { binary attributes and } \\
13 \text { multi-class attributes }\end{array}$ & outdoor & http://www.vision.caltech.edu/ dhall/projects/CRP/ \\
\hline CAD [38] & 1856 & $\begin{array}{c}23 \text { binary attributes and } 3 \\
\text { multi-class attributes }\end{array}$ & $\begin{array}{c}\text { image site } \\
\text { Sartorialist* and Flickr }\end{array}$ & https://purl.stanford.edu/tb980qz1002 \\
\hline BAP [8] & 8035 & 9 binary attributes & $\begin{array}{c}\text { H3D [46] dataset } \\
\text { PASCAL VOC } 2010 \text { [47] }\end{array}$ & https://www2.eecs.berkeley.edu/Research/Projects/CS/vision/shape/poselets/ \\
\hline
\end{tabular}

and 17661 images respectively. The attributes are annotated in the identity level, every image in this dataset is annotated with 23 attributes.

CRP [42] Every image in the CRP dataset was captured in the wild, pedestrians were recorded "in-the-wild" from a moving vehicle. The CRP dataset contains 7 videos and 27454 pedestrian bounding boxes. Each pedestrian was labelled with four types of attributes, age ( 5 classes), sex ( 2 classes), weight (3 classes) and clothing type (4 classes). This dataset is split into a training/validation set containing 4 videos, with the remaining 3 videos forming the test set.

Clothing Attributes Dataset, CAD [28] The Clothing Attributes Dataset was collected from Sartorialist ${ }^{\dagger}$ and Flickr. The dataset contains 1856 images, with 26 ground truth clothing attributes collected using Amazon Mechanical Turk. All labels are arranged in the order from image 1 to 1856 . Some label entries are "NaN", meaning the 6 human workers cannot reach an agreement on this clothing attribute. There are 26 attributes in total, including 23 binary-class attributes ( 6 for pattern, 11 for color and 6 miscellaneous attributes) and 3 multi-class attributes (sleeve length, neckline shape and clothing category). This dataset was split by leave-1-out for training and test.

\subsection{Evaluation Criteria}

zhu et al. [40] evaluate the performance of each attribute classification with the Receiver Operating Characteristic (ROC) and the Area Under the average ROC Curve (AUC) which are calculated by two indicators, the recall rate and false positive rate. The recall rate is the fraction of the correctly detected positives over the total amount of positive samples, and the false positive rate means the fraction of the mis-classified negatives out of the whole negative samples. At various threshold settings, a ROC curve can be drawn by plotting the recall rate vs. the false positive rate. In addition, the Area Under the average ROC Curve (AUC) is also used by zhu et al. [40] so as to make a clearer performance comparison.

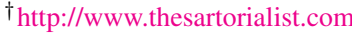

Deng at al. [34] adopt the mean acccuracy (mA) to evaluate the attribute recognition algorithms. For each attribute, $\mathrm{mA}$ calculates the classification accuracy of positive and negative samples respectively, and then gets their average values as the recognition result for the attribute. Finally, a recognition rate is obtained by taking an average over all attributes. The evaluation criterion can be calculated through the following formula:

$$
m A=\frac{1}{2 N} \sum_{i=1}^{L}\left(\frac{T P_{i}}{P_{i}}+\frac{T N_{i}}{N_{i}}\right)
$$

where $\mathrm{L}$ is the number of attributes. $T P_{i}$ and $T N_{i}$ are the number of correctly predicted positive and negative examples respectively, $P_{i}$ and $N_{i}$ are the number of positive and negative examples respectively.

The above evaluation criterions treats each attribute independently and ignores the inter-attribute correlation which exists naturally in multi-attribute recognition problem. Li at al. [35] call the above solution as label-based evaluation criterions and propose using the example-based evaluation criterions inspired by a fact that example-based evaluation captures better the consistence of prediction on a given pedestrian image [51]. The examplebased evaluation criteria which widely used include four metrics: accuracy, precision, recall rate and F1 value, as defined below:

$$
\begin{gathered}
A c c_{\text {exam }}=\frac{1}{N} \sum_{i=1}^{N} \frac{\left|Y_{i} \cap f\left(x_{i}\right)\right|}{\left|Y_{i} \cup f\left(x_{i}\right)\right|} \\
\operatorname{Prex}_{\text {exam }}=\frac{1}{2 N} \sum_{i=1}^{N} \frac{\left|Y_{i} \cap f\left(x_{i}\right)\right|}{\left|f\left(x_{i}\right)\right|} \\
\operatorname{Rec}_{\text {exam }}=\frac{1}{2 N} \sum_{i=1}^{N} \frac{\left|Y_{i} \cap f\left(x_{i}\right)\right|}{\left|Y_{i}\right|} \\
F 1=\frac{2 * \operatorname{Prec}_{\text {exam }} * \operatorname{Rec}_{\text {exam }}}{\operatorname{Prec}_{\text {exam }}+\operatorname{Rec}_{\text {exam }}}
\end{gathered}
$$

where $N$ is the number of examples, $Y_{i}$ is the ground truth positive labels of the $i$-th example, $f(x)$ returns the predicted 
positive labels for $i$-th example. And $|\cdot|$ means the set cardinality.

\section{Regular Pipeline for Par}

The pedestrian attributes in practical video surveillance may contain dozens of categories, as defined in many popular benchmarks. Learning each attribute independently is one intuitive idea, but it will make the PAR redundant and inefficiency. Therefore, the researchers prefer to estimate all the attributes in one model and treat each attribute estimation as one task. Due to the elegant and efficient of multi-task learning, it draw more and more attentions. On the another hand, the model takes the given pedestrian image as input and output corresponding attributes. PAR also belongs to the domain of multi-label learning. In this section, we will give a brief introduction on regular pipeline for pedestrian attribute recognition from these two aspects, i.e., the multi-label learning and multi-task learning.

\subsection{Multi-task Learning}

To handle one specific task in the machine learning community, a traditional solution is to design a evaluation criterion, extract related feature descriptors and construct single or ensemble models. It use the feature descriptors to optimize the model parameters and achieve best results according to evaluation criterion to improve the overall performance. This pipeline may achieve satisfying results on single task, however, it ignore the other tasks which may bring further improvements for the evaluation criterion.

In the real world, many things are correlated. The learning of one task may relies on or constrain the others. Even one task is decomposed, but the sub-tasks still have correlations to some extent. Process single task independently is prone to ignore such correlations, thus, the improvement of final performance may meet the bottleneck. Specifically, the pedestrian attributes are correlated with each other, such as the gender and cloth style. On the other hand, the supervised learning needs massive annotated training data which is hard to collect. Therefore, the most popular approach is to joint learning multi-tasks to mine the shared feature representation. It has been widely applied in multiple domains, such as natural language processing, computer vision.

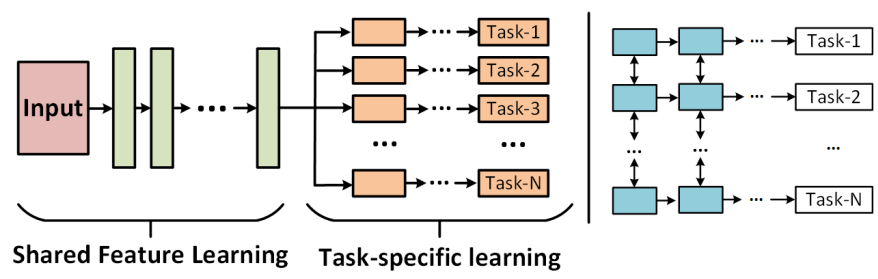

Figure 4. The illustration of Hard (left sub-figure) and Soft (right subfigure) parameter sharing for multi-task learning in deep neural networks [52].

With the development of deep learning, many efficient algorithms are proposed by integrating multi-task learning and deep neural networks. To fully understand the reasons behind the efficiency of MTL, we need to analyse its detailed mechanism. According to the study of Ruder et al. [52], the reasons can be concluded as following five points: implicit data augmentation, attention focusing, eavesdropping, representation bias, regularization. For the details of these reasons, please refer to their original paper. Generally speaking, there are two kinds of approaches in deep learning based multi-task learning, i.e. the hard and soft parameter sharing. The hard parameter sharing usually take the shallow layers as shared layers to learn the common feature representations of multiple tasks, and treat the high-level layers as taskspecific layers to learn more discriminative patterns. This mode is the most popular framework in the deep learning community. The illustration of hard parameter sharing can be found in Figure 4 (left sub-figure). For the soft parameter sharing multi-task learning (as shown in Figure 4 (right sub-figure)), they train each task independently, but make the parameters between different tasks similar via the introduced regularization constrains, such as $L_{2}$ distance [53] and trace norm [54].

Therefore, it is rather intuitive to apply the multi-task learning for pedestrian attribute recognition and many algorithms are also proposed based on this framework [5] [6] [6] [7] [8] [9] [10] [11].

\subsection{Multi-label Learning}

For the multi-label classification algorithms, the following three kinds of learning strategy can be concluded as noted in [51]: 1). First-order strategy, is the simplest form and could directly transform the multi-class into multiple binary-classification problem. Although it achieve better efficiency, but this strategy can not model the correlations between multi-labels which leads to bad generic; 2). Second-order strategy, takes the correlations between each label pair and achieves better performance than first-order strategy; 3). High-order strategy, considers all the label relations and implement multi-label recognition system by modelling the influence of each label on others. This approach is generic but with high complexity which may weak at process large-scale image classification task. Therefore, the following two approaches are frequently used for model construction: i.e. the problem transformation and algorithm adaptation. The visualization of some representative multi-label learning algorithms can be found in Figure 5, as noted in [51].

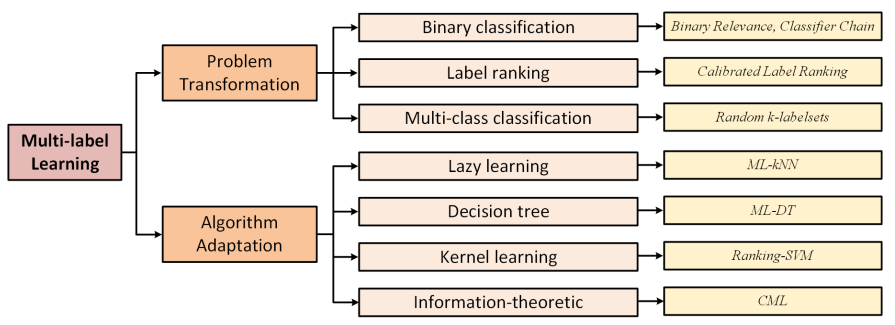

Figure 5. Categorization of representative multi-label learning algorithms reviewed in [51].

To simplify the multi-label classification using problem transformation, existing widely used frameworks can be adopted. Some representative algorithms are: 1). binary relevance algorithm [55], which directly transform the multi-label into multiple binary classification problem and finally fuse all the binary classifiers together for multi-label classification. This approach is simple and intuitive, but neglect the correlations between multiple labels; 2). classifier chain algorithm [56], the basic idea of this algorithm is to transform the multi-label learning problem into binary classification chain. Each binary classifier is depend on its previous one in the chain; 3). calibrated label ranking algorithm [57], which considers the correlations between paired labels and transforms the multi-label learning into label ranking problem; 4). random $k$-Labelsets algorithm [58], it transforms the multi-label 
classification problem into sets of multiple classification problems, the classification task in each set is a multi-class classifier. And the categories the multi-class classifiers need to learning is the subset of all labels.

Different from problem transformation, the algorithm adaptation directly improve existing algorithm and apply on multilabel classification problem, including: 1). multi-label k-nearest neighbour, $M L-k N N$ [59], adopt the kNN techniques to handle the multi-class data and utilize the maximum a posteriori (MAP) rule to make prediction by reasoning with the labelling information embodied in the neighbours. 2). multi-label decision tree, $M L-D T$ [60], which attempt to deal with multi-label data with decision tree, an information gain criterion based on multi-label entropy is utilized to build the decision tree recursively. 3). ranking support vector machine, Rank-SVM [61], adopt the maximum margin strategy to handle this problem, where a set of linear classifiers are optimized to minimize the empirical ranking loss and enabled to handle non-linear cases with kernel tricks. 4). collective multilabel classifier, CML [62], adapt maximum entropy principle to deal with multi-label task, where correlations among labels are encoded as constraints that the resulting distribution must satisfy.

The regular pipeline of multi-label pedestrian attribute recognition can be found in Figure 6. The machine learning model takes the human image (optionally pre-processed) as input and extract its feature representation (such as HOG, SIFT or deep features). Some commonly used pre-processing techniques are normalization, random cropping, whitening process, etc. It target at improve the quality of input image, suppress the unnecessary deformation or augment the image features which may important for subsequent operations, to improve the generic of the trained model. after that, they train a classifier based on extracted features to predict corresponding attributes. Existing deep leaning based PAR algorithm could jointly learn the feature representation and classifier in an end-to-end manner which significantly improve the final recognition performance.

\section{Deep Neural Networks}

In this subsection, we will review some well-known network architectures in deep learning community which are already or maybe used for the pedestrian attribute recognition task.

LeNet [63] is first proposed by Yann LeCun et al. in 1998. It is first designed for handwritten and machine-printed character recognition, as shown in the website ${ }^{\ddagger}$. The architecture of LeNet can be found in Figure 7 . It takes $32 \times 32$ single channel images as inputs and use 2 groups convolutional + max pooling layers to extract its features. The classification is done by 2 fully-connected layers and output the distribution of numbers.

AlexNet [64] is a milestone in deep learning history which is proposed by Alex et al. in 2012 and win the ILSVRC-2012 with a TOP-5 test accuracy of $84.6 \%$. AlexNet was much larger than previous CNNs used for computer vision tasks, such as, LeNet. It has 60 million parameters and 650,000 neurons, as shown in Figure 8. It consists of 5 convolutional layers, max-pooling layers, Rectified Linear Units (ReLUs) as non-linearities, three fullyconnected layers, and dropout unit.

VGG [65] is a CNN model proposed by the Visual Geometry Group (VGG) from the University of Oxford. This network use more convolutional layers (16, 19 layers) and also achieve good

$\ddagger$ http://yann.lecun.com/exdb/lenet/ results on ILSVRC-2013. Many subsequent neural networks all follow this network. It first use a stack of convolutional layers with small receptive fields in the first layers while previous networks are adopt layers with large receptive fields. The smaller receptive fields can reduce the parameters by a wide margin and more nonlinearities, and making the learned features more discriminative and also more efficient to training.

GoogleNet [67] is another popular network architecture in deep learning community (22 layers). Different from traditional sequential manner, this network first introduce the concept of inception module and win the competition of ILSVRC-2014. See Figure 10 for details. The GoogleNet contains a Network in Network (NiN) layer [68], a pooling operation, a large-sized convolution layer, and a small-sized convolution layer. These layers can be computed in parallel and followed by $1 \times 1$ convolution operations to reduce dimensionality.

Residual Network [66] is first well known by its super deep architecture (more than $1 \mathrm{k}$ layers) while previous network are rather "shallow" by comparison. The key contribution of this network is the introduction of residual blocks, as shown in Figure 11. This mechanism can address the problem of training a really deep architecture by introducing identity skip connections and copy their inputs to the next layer. The vanishing gradients problem can be handled to a large extent with this method.

Dense Network [69] is proposed by Huang et al. in 2017. This network further extend the idea of residual network and have better parameter efficiency, one big advantage of DenseNets is their improved flow of information and gradients throughout the network, which makes them easy to train. Each layer has direct access to the gradients from the loss function and the original input signal, leading to an implicit deep supervision [70]. This helps training of deeper network architectures.

Capsule Network [71] [72] is introduced in 2017 by Hiton et al. to handle the limitations of standard CNN. As we all known the use of max pooling layers in standard $\mathrm{CNN}$ reduced the dimension of feature maps generated from previous convolutional layers and make the feature learning process more efficient. As shown in Figure 13 (b), the two face images are similar to a CNN due to they both contain similar elements. However, standard CNN can not capture the difference between the two face images since the usage of max pooling layers. The capsule network is proposed to handle this issue by abandon the max pooling layers and use a capsule to output a vector instead of a value for each neurons. This make it possible to use a powerful dynamic routing mechanism ("routingby-agreement") to ensure that the output of the capsule gets sent to an approximate parent in the layer above. The utilization of margin loss and re-construction loss for the training of capsule network validated its effectiveness. And some ablation studies also demonstrate the attributes of each digit can be encoded in the output vectors by capsule network.

Graph Convolutional Network [73] attempt to extend the $\mathrm{CNN}$ into non-grid data due to the standard convolution operation on images/videos can not be directly used in graph structured data. The goal of GCN is to learn a function of signals/features on a graph $G=(V, E)$ which takes feature description $x_{i}$ for each node $i$ and representative description of the adjacency matrix $\mathbf{A}$ as input, and produces a node-level output $\mathbf{Z}$. The overall architecture of GCN can be found in Figure 14.

ReNet [74] In order to extend Recurrent Neural Networks (RNNs) architectures to multi-dimensional tasks, Graves et al. [75] proposed a Multi-dimensional Recurrent Neural Network 


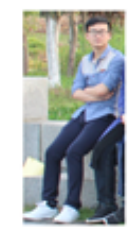

Input Image



Feature (data augmentation)

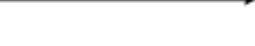

(n)

Figure 6. The pipeline for regular pedestrian attributes recognition algorithm.

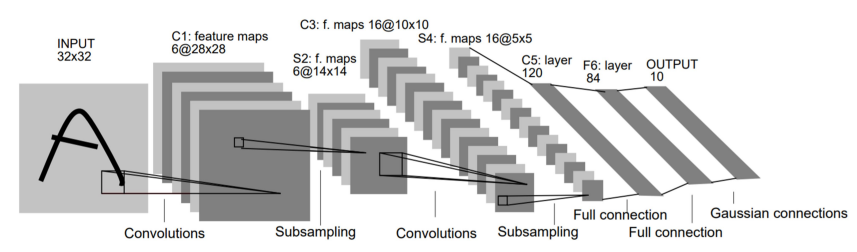

Figure 7. Architecture of LeNet-5, a Convolutional Neural Network, here for digits recognition. Each plane is a feature map, i.e. a set of units whose weights are constrained to be identical.

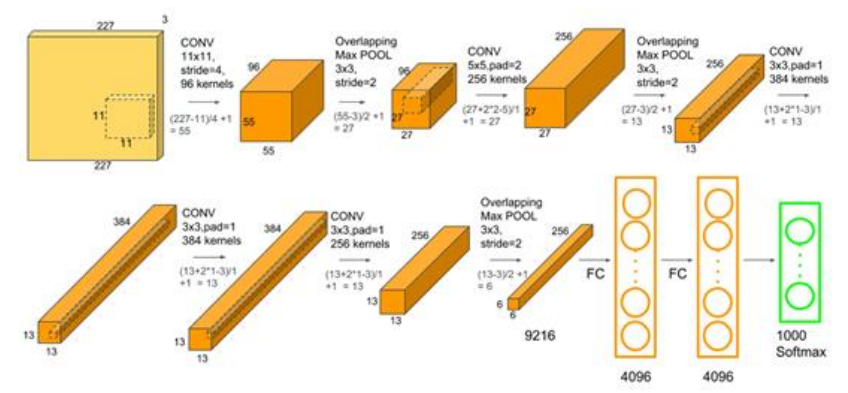

Figure 8. Architecture of AlexNet for ImageNet classification. This figure is taken from: https://www.learnopencv.com/understanding-alexnet/.

(MDRNN) architecture which replaces each single recurrent connection from standard RNNs with $d$ connections, where $d$ is the number of spatio-temporal data dimensions. Based on this initial approach, Visin el al. [74] proposed ReNet architecture in which instead of multidimensional RNNs, they have been using usual sequence RNNs. In this way, the number of RNNs is scaling linearly at each layer regarding to the number of dimensions $d$ of the input image (2d). In this approach, each convolutional layer (convolution + pooling) is replaced with four RNNs sweeping the image vertically and horizontally in both directions as we can see in Figure 15.

Recurrent Neural Network, RNN. Traditional neural network is based on the assumption that all inputs and outputs are independent of each other, however the assumption may not true in many tasks, such as sentence translation. Recurrent Neural Network (RNN) is proposed to handle the task which involve sequential information. RNNs are called recurrent because they perform the same task for every element of a sequence, with the output being depended on the previous computations. Another way to think about RNNs is that they have a "memory" which captures information about what has been calculated so far. In theory RNNs can make use of information in arbitrarily long sequences, but in practice they are limited to looking back only a few steps.

Long Short-term Memory, LSTM is introduced to handle

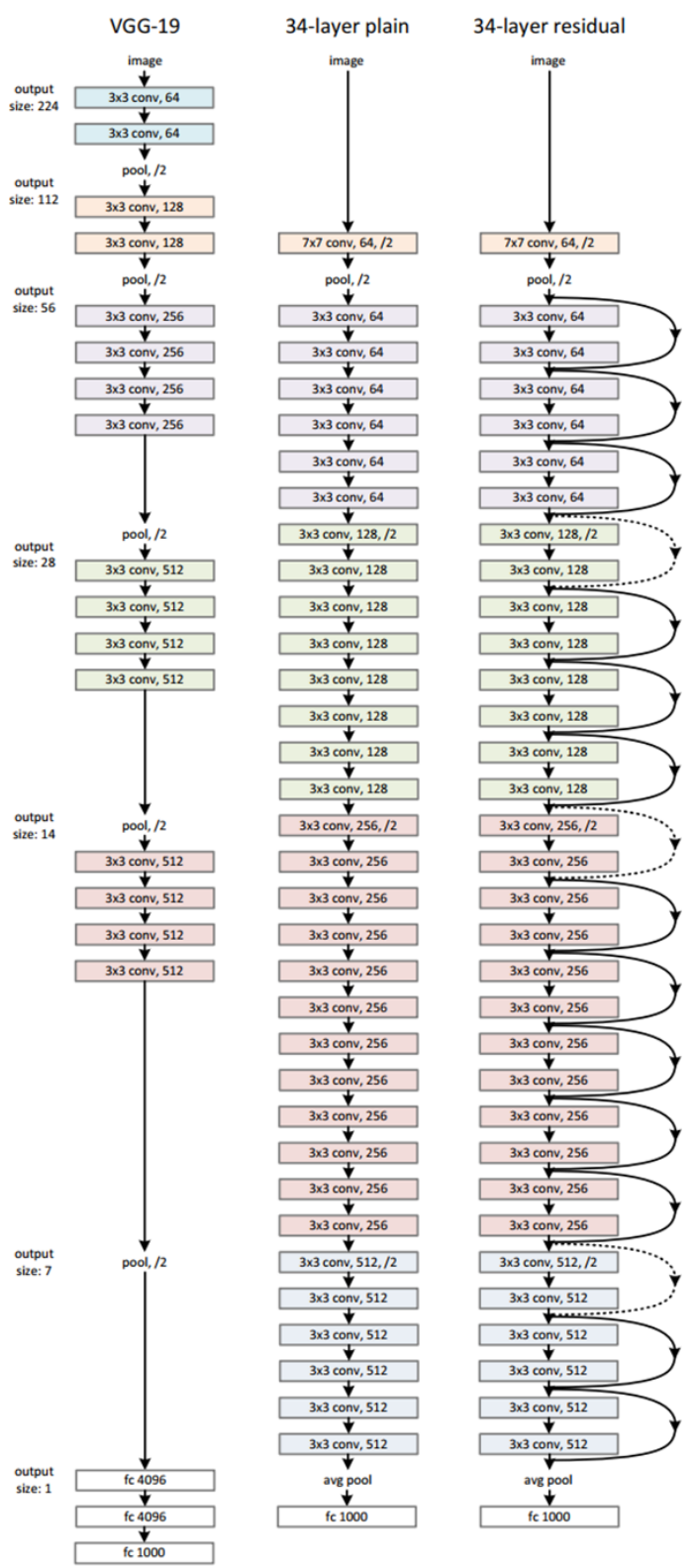

Figure 9. Architecture of VGG-19, 34-layer plain and Residual Network34 for ImageNet classification. This figure is taken from the paper of Residual Network [66].

the issue of gradient vanish or explosion of RNN. An LSTM has three of these gates, to protect and control the cell state, i.e. the forget gate, input gate and output gate. Specifically, we denote the input sequences as $\mathbf{X}=\left(\mathbf{x}_{1}, \mathbf{x}_{2}, \ldots, \mathbf{x}_{N}\right)$. At each position 

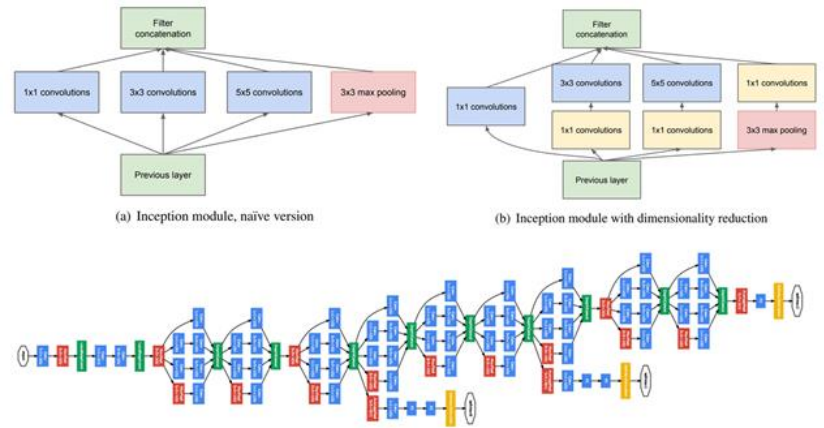

(c) Architecture of GoogleNet

Figure 10. Architecture of GoogleNet. This figure is taken from the paper of GoogleNet [67].

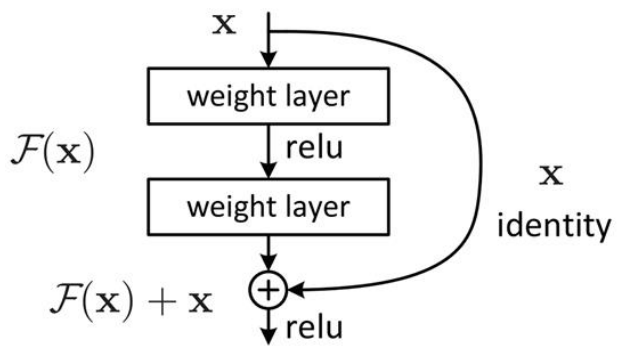

Figure 11. Residual learning: a building block. This figure is taken from the paper of Residual Network [66].

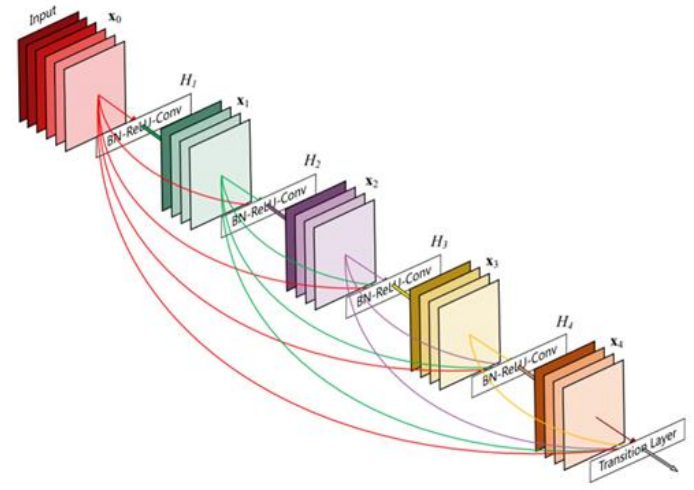

Figure 12. A 5-layer dense block with a growth rate of $k=4$. Each layer takes all preceding feature-maps as input. This figure is taken from the paper of DenseNet [69].

$k, k \in[1, N]$, there is a set of internal vectors, including an input gate $\mathbf{i}_{k}$, a forget gate $\mathbf{f}_{k}$, an output gate $\mathbf{o}_{k}$ and a memory cell $\mathbf{c}_{k}$. The hidden state $\mathbf{h}_{k}$ can be computed by all these vectors, as follows:

$$
\begin{aligned}
\mathbf{i}_{k} & =\sigma\left(\mathbf{W}^{i} \mathbf{x}_{k}+\mathbf{V}^{i} \mathbf{h}_{k-1}+\mathbf{b}^{i}\right), \\
\mathbf{f}_{k} & =\sigma\left(\mathbf{W}^{f} \mathbf{x}_{k}+\mathbf{V}^{f} \mathbf{h}_{k-1}+\mathbf{b}^{f}\right), \\
\mathbf{o}_{k} & =\sigma\left(\mathbf{W}^{o} \mathbf{x}_{k}+\mathbf{V}^{o} \mathbf{h}_{k-1}+\mathbf{b}^{o}\right), \\
\mathbf{c}_{k} & =\mathbf{f}_{k} \odot \mathbf{c}_{k-1}+\mathbf{i}_{k} \odot \tanh \left(\mathbf{W}^{c} \mathbf{x}_{k}+\mathbf{V}^{c} \mathbf{h}_{k-1}+\mathbf{b}^{c}\right), \\
\mathbf{h}_{k} & =\mathbf{o}_{k} \odot \tanh \left(\mathbf{c}_{k}\right)
\end{aligned}
$$

where $\sigma$ is the sigmoid function, $\odot$ is the element-wise multiplication of two vectors, and all $\mathbf{W}^{*}, \mathbf{V}^{*}, \mathbf{b}^{*}$ are weight matrices

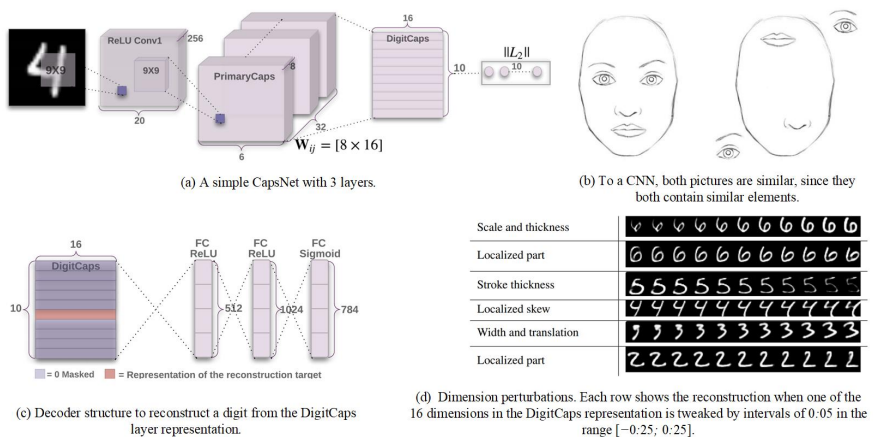

Figure 13. The motivation and illustration of Capsule Network. This figure is taken from the paper of Capsule Network [71] and the blog https://medium.com/ai\%C2\%B3-theory-practice-business/ understanding-hintons-capsule-networks-part-i-intuition-b4b559d1159b.



Figure 14. The illustration of Graph Convolutional Network (GCN). This figure is rewritten based on the blog http://tkipf.github.io/ graph-convolutional-networks/

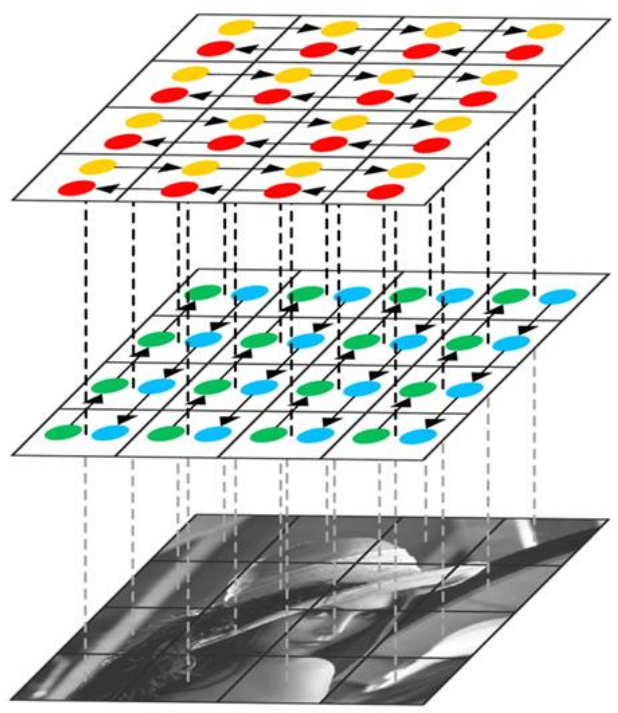

Figure 15. One layer of ReNet architecture modeling vertical and horizontal spatial dependencies. This figure is adopted from [74].

and vectors to be learned. Please see Figure 17 (a) for detailed information of LSTM.

GRU. A slightly more dramatic variation on the LSTM is the Gated Recurrent Unit, or GRU, introduced by [76]. It combines the forget and input gates into a single update gate. It also merges the cell state and hidden state, and makes some other changes. The 


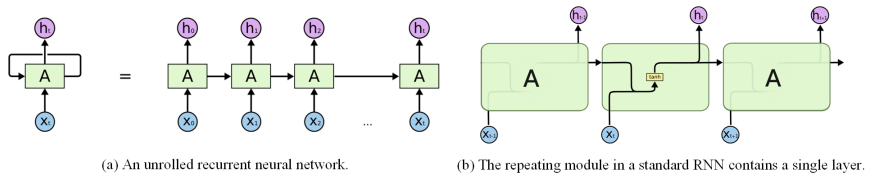

Figure 16. The illustration of RNN. This figure is adopted from http:// colah.github.io/posts/2015-08-Understanding-LSTMs/.

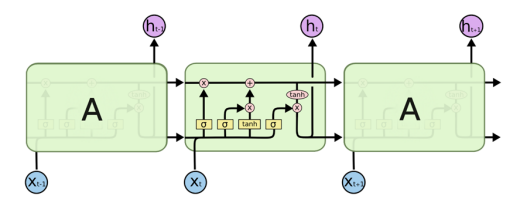

(a) The repeating module in an LSTM contains four interacting layers.

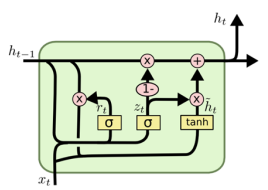

(b) The GRU Unit
Figure 17. The illustration of LSTM and GRU Unit. This figure is adopted from http://colah.github.io/posts/2015-08-Understanding-LSTMs/.

resulting model is simpler than standard LSTM models, and has been growing increasingly popular. The detailed GRU Unit can be found in Figure 17 (b).

Recursive Neural Network (RvNN) [77] As noted in §, a recursive neural network is a kind of deep neural network created by applying the same set of weights recursively over a structured input, to produce a structured prediction over variable-size input structures, or a scalar prediction on it, by traversing a given structure in topological order. RvNNs have been successful, for instance, in learning sequence and tree structures in natural language processing, mainly phrase and sentence continuous representations based on word embedding. The illustration of RvNN can be found in Figure 18.

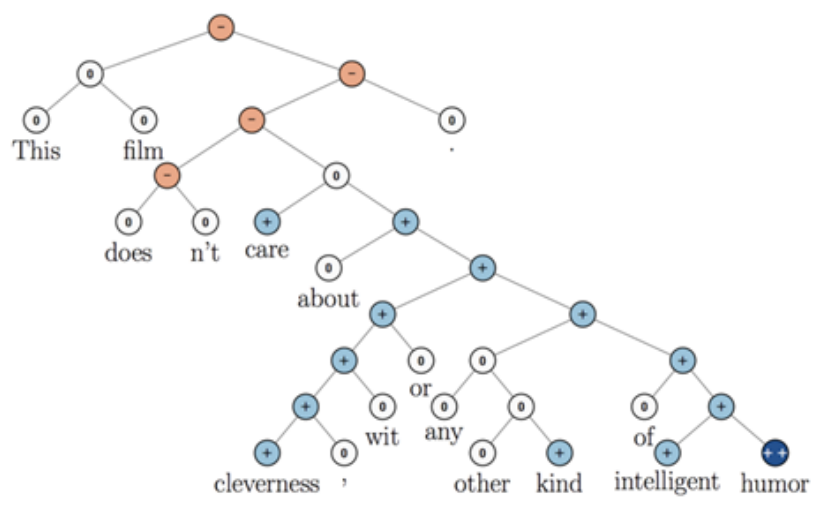

Figure 18. The illustration of Recursive Neural Network (RvNN).

Sequential CNN. [78], [79] Different from regular works which use RNN to encode the time series inputs, the researchers also study $\mathrm{CNN}$ to achieve more efficient operation. With the sequential CNN, the computations over all elements can be fully parallelized during training to better exploit the GPU hardware and optimization is easier since the number of non-linearities is fixed and independent of the input length [78].

External Memory Network. [81] Visual attention mechanism can be seen as a kind of short-term memory that allocates attention over input features they have recently seen, while external memory network could provide long-term memory through the read and

\footnotetext{
$\S$ https://en.wikipedia.org/wiki/Recursive_neural_network
}

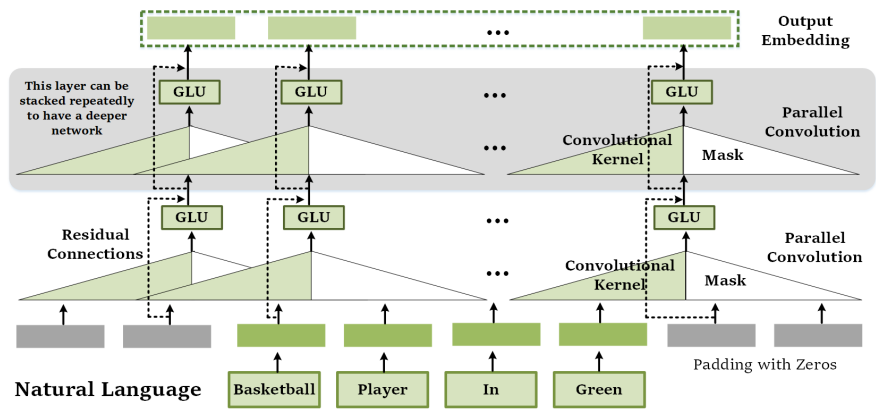

Figure 19. The illustration of all convolutional network for sequential modeling. This figure is adopted from [80].

write operation. It has widely used in many applications such as visual tracking [82], visual question answering [83], [84].

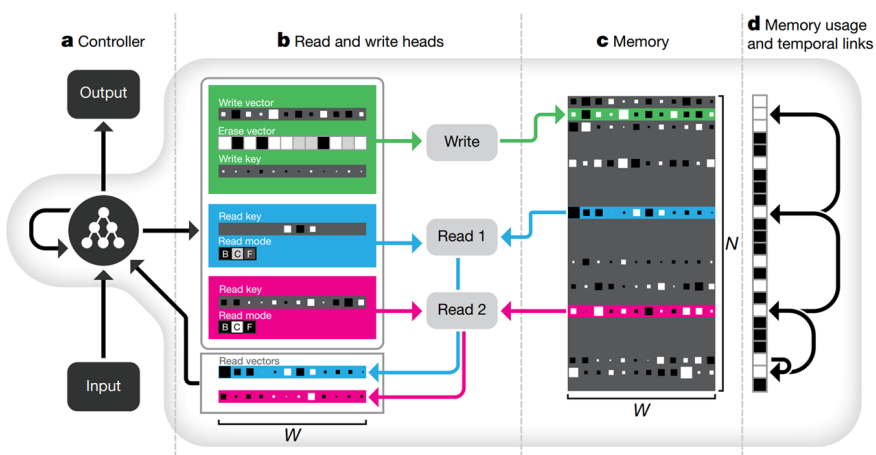

Figure 20. The illustration of external memory network. This figure is adopted from DNC [81].

Deep Generative Model. In recent years, deep generative models achieved a great development and many popular algorithms has been proposed, such as VAE (variational auto-encoder) [85], GAN (generative adversarial networks) [86], CGAN (conditional generative adversarial network) [87]. The illustration of these three models can be found in Fig. 21. We think the strategy of attribute based pedestrian image generation can handle the issue of low resolution, unbalanced data distribution and augment the training dataset significantly.

\section{The Review of PAR Algorithms}

In this section, we will review the deep neural network based PAR algorithms from following eight aspects: global based, local parts based, visual attention based, sequential prediction based, new designed loss function based, curriculum learning based, graphic model based and others algorithms.

\subsection{Global Image-based Models}

In this section, we will review the PAR algorithms which consider global image only, such as ACN [5], DeepSAR [6], DeepMAR [6], MTCNN [7].

\subsubsection{ACN (ICCVW-2015) [5]}

This paper propose a multi-branch classification layers for each attribute learning with convolutional network. As shown in Fig. 22, they adopt a pre-trained AlexNet as basic feature extraction 



Figure 21. The illustration of VAE, GAN and CGAN.

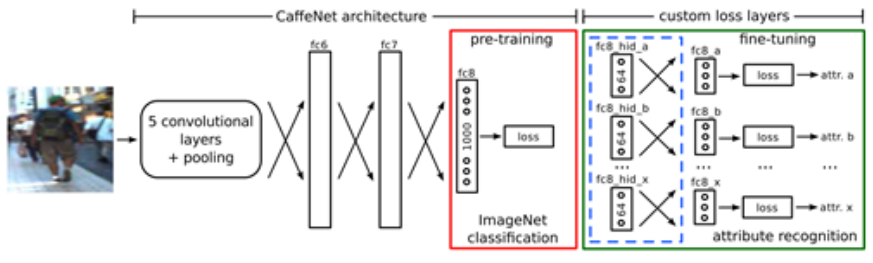

Figure 22. The illustration of ACN [5].

sub-network, and replace the last fully connected layer with one loss per attribute using the KL-loss (Kullback-Leibler divergence based loss function). The specific formulation can be described as follows:

$$
K L(P \| Q)=\sum_{i}^{N} P\left(x_{i}\right) \log \frac{Q\left(x_{i}\right)}{P\left(x_{i}\right)}
$$

where $Q$ is the neural network's prediction and $P$ is the binary attribute's state in reality.

In addition, they also propose a new dataset named as PARSE$27 \mathrm{k}$ to support their evaluation. This dataset contains 27000 pedestrians and annotated with 10 attributes. Different from regular person attribute dataset, they propose a new category annotation, i.e., not decidable (N/A). Because for most input images, some attributes are not decidable due to occlusion, image boundaries, or any other reason.

\subsubsection{DeepSAR and DeepMAR (ACPR-2015) [6]}
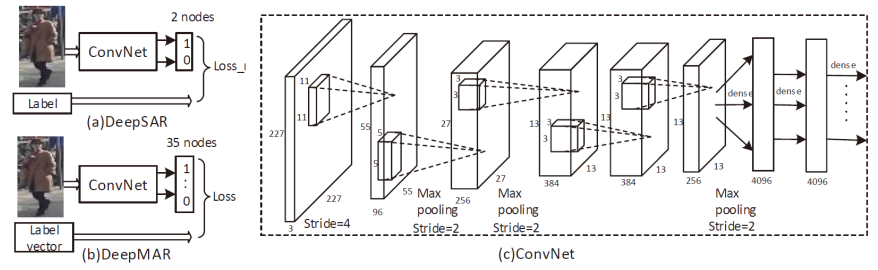

Figure 23. The overall pipeline of DeepSAR and DeepMAR [6].

This paper introduce deep neural network for person attribute recognition and attempt to handle the following two issues existed in traditional methods: 1). hand crafted features used in existing methods, like HOG, color histograms, LBP (local binary patterns); 2). correlations between attributes are usually ignored. The authors propose two algorithms i.e. DeepSAR and DeepMAR in this paper, as shown in Fig. 23. They adopt AlexNet as their backbone network and obtain the DeepSAR by changing the output category defined in last dense layer into two. The softmax loss is adopted to compute final classification loss.
Although the DeepSAR can use deep features for binary classification. However, it did not model the correlations between human attributes which maybe the key to further improve the overall recognition performance. Therefore, they propose the DeepMAR which takes human image and its attribute label vectors simultaneously and jointly considers all the attributes via sigmoid cross entropy loss:

$$
\begin{gathered}
L_{c e}=-\frac{1}{N} \sum_{i=1}^{N} \sum_{l=1}^{L} y_{i l} \log \left(\hat{P_{i l}}\right)+\left(1-y_{i l}\right) \log \left(1-\hat{p_{i l}}\right) \\
\hat{p_{i l}}=\frac{1}{1+\exp \left(-x_{i l}\right)}
\end{gathered}
$$

where $\hat{p_{i l}}$ is the estimated score for the $l$ 'th attribute of sample $x_{i}$. $y_{i l}$ is the ground truth label.

In addition, they also consider the unbalanced label distribution in practical surveillance scenarios and propose an improved loss function as follows:

$$
\begin{gathered}
L_{w c e}=-\frac{1}{N} \sum_{i=1}^{N} \sum_{l=1}^{L} w_{l}\left(y_{i l} \log \left(\hat{P_{i l}}\right)+\left(1-y_{i l}\right) \log \left(1-\hat{p_{i l}}\right)\right) \\
w_{l}=\exp \left(-p_{l} / \sigma^{2}\right)
\end{gathered}
$$

where $w_{l}$ is the loss weight for the $l^{t h}$ attribute. $p_{l}$ denote the positive ratio of $l^{t h}$ attribute in the training dataset. $\sigma$ is a hyper parameter.

\subsubsection{MTCNN (TMM-2015) [7]}

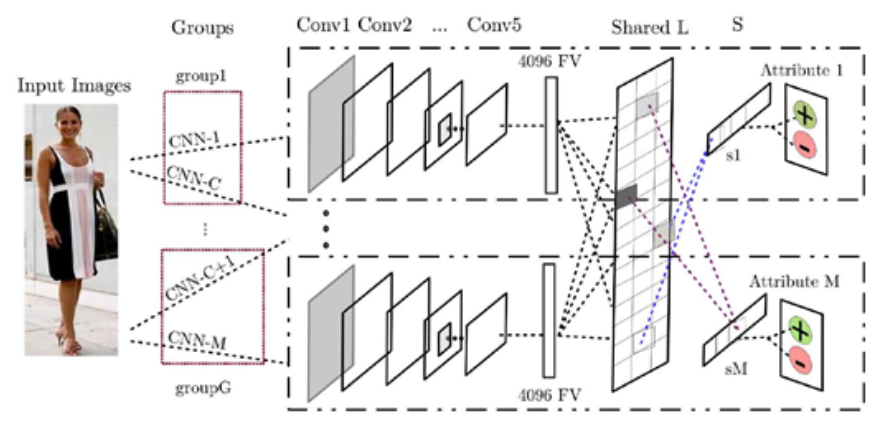

Figure 24. The pipeline of Mutli-task CNN [7].

This paper propose a joint multi-task learning algorithm for attribute estimation using CNN, named MTCNN, as shown in Fig. 24. The MTCNN let the CNN models to share visual knowledge among different attribute categories. They adopt multi-task learning on the $\mathrm{CNN}$ features to estimate corresponding attributes. 
In their MTL framework, they also use rich information groups because knowing any a priori information about the statistical information of features will definitely aid the classifiers. They use decomposition method to obtain shareable latent task matrix $L$ and combination matrix $S$ from total classifier weights matrix $W$, and thus flexible global sharing and competition between groups through learning localized features, i.e., $W=L S$. Therefore, the objective function (named MTL squared maxing hinge loss) is formulated as follows:

$$
\begin{array}{r}
\min _{L, S} \sum_{m=1}^{M} \sum_{i=1}^{N_{m}} \frac{1}{2}\left[\max \left(0,1-Y_{m}^{i}\left(L s^{m}\right)^{T} X_{m}^{i}\right)\right]^{2}+ \\
\mu \sum_{k=1}^{K} \sum_{g=1}^{G}\left\|s_{k}^{g}\right\|_{2}+\gamma\|L\|_{1}+\lambda\|L\|_{F}^{2}
\end{array}
$$

where $\left(X_{m}^{i}, Y_{m}^{i}\right)_{i=1}^{N_{m}}$ is the training data, $N_{m}$ is the number of training samples of the $m^{\text {th }}$ attribute. $K$ is the total latent task dimension space. The model parameter of $m^{t h}$ attribute category is denoted as $L s^{m}$.

They employ the Accelerate Proximal Gradient Descent (APG) algorithm to optimize both $L$ and $S$ in an alternating manner. Therefore, the overall model weight matrix $W$ can be obtain after obtain the $L$ and $S$.

Summary: According to the aforementioned algorithms [5] [6] [6] [7], we can find that these algorithms all take the whole images as input and conduct multi-task learning for PAR. They all attempt to learn more robust feature representations using feature sharing, end-to-end training or multi task learning squared maxing hinge loss. The benefit of these models are simple, intuitive and highly efficient which are very important for practical applications. However, the performance of these models is still limited due to the lack of consideration of fine-grained recognition.

\subsection{Part-based Models}

In this subsection, we will introduce the part-based algorithms which could joint utilize local and global information for more accurate PAR. The algorithms are including: Poselets [8], RAD [9], PANDA [10], MLCNN [11], AAWP [12], ARAP [13], DeepCAMP [14], PGDM [15], DHC [16], LGNet [17].

\subsubsection{Poselets (ICCV-2011) [8]}

The motivation of this paper is that we can train attribute classifiers simpler if we could isolate image patches corresponding to the same body part from the same viewpoint. However, directly use object detectors is not reliable for body parts localization at that time (before the year of 2011) due to its limited ability. Therefore, the authors adopt the poselets [46] to decompose the image into a set of parts, each capturing a salient pattern corresponding to a given viewpoint and local pose. This provide a robust distributed representation of a person from which attributes can be inferred without explicitly localizing different body parts.

The Fig. 25 illustrate the overall pipeline of Poselets. Specifically speaking, they first detect the poselets on given image and obtain their joint representations by concatenating the HOG, color histogram and skin-mask features. Then, they train multiple SVM classifiers which are used for poselet-level, person-level, context-level attribute classification, respectively. The poselet-level classifiers target at determine the presence of an attribute from a given part of the person under a given viewpoint. The personlevel classifiers are used to combine the evidence from all body

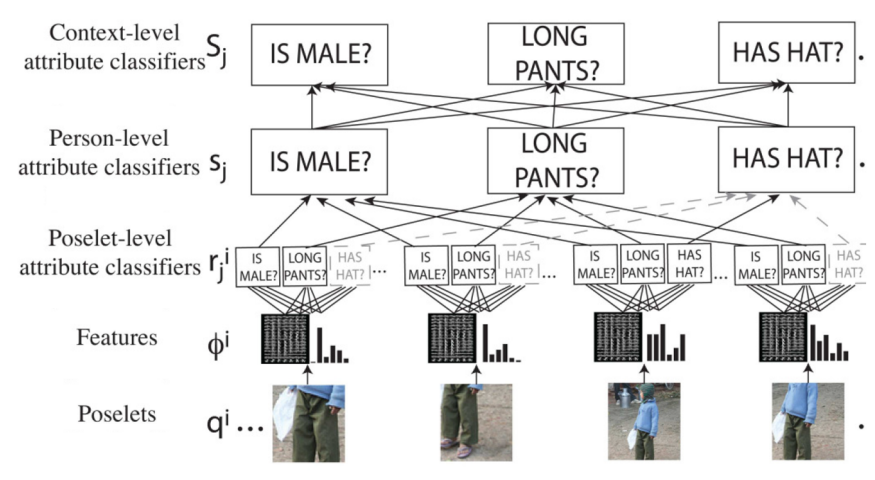

Figure 25. The overview of Poselets at test time [8].

parts and the context-level classifiers take the output of all personlevel classifiers as input and try to exploit the correlations between the attributes. Their attribute prediction results is the output of context-level classifiers.

The idea of poselets is also extended by combining it with deep neural network [88], named deep poselets. It can be used for human body parts localization based tasks, such as human detection task [89].

\subsubsection{RAD (ICCV-2013) [9]}

This paper propose a part learning algorithm from the perspective of appearance variance while previous works focus on handling geometric variation which require manual part annotation, such as poselet [8]. They first divide the image lattice into a number of overlapping subregions (named window). As shown in Fig. 26 (a), a grid of size $W \times H$ is defined and any rectangle on the grid containing one or more number of cells of the grid forms a window. The proposed method is more flexibility in shape, size and location of part window while previous works (such as spatial pyramid matching structure, SPM [90]) recursively divides the region into four quadrants and make all subregions are squares that do not overlap with each other at the same level.

With all these windows, they learn a set of part detectors that are spatially associated with that particular window. For each window, all corresponding image patches are cropped from training images and represented by HOG [1] and color histogram feature descriptors. Then, K-means clustering is conducted based on the extracted features. Each obtained cluster denotes a specific appearance type of a part. They also train a local part detector for each cluster by logistic regression as a initial detector and iteratively refine it by applying it onto the entire set again and updating the best location and scale to handle the issue of noisy clusters.

After learning the parts at multi-scale overlapping windows, they follow the method for attribute classification proposed in the Poselet-based approach [8]. Specifically, they aggregate the scores from these local classifiers with the weights given by part detection scores for final prediction.

\subsubsection{PANDA (CVPR-2014) [10]}

Zhang et al. find the signal associated with some attributes is subtle and the image is dominated by the effects of pose and viewpoint. For the attribute of wear glasses, the signal is weak at the scale of the full person and the appearance varies significantly with the head pose, frame design and occlusion by the hair. They 


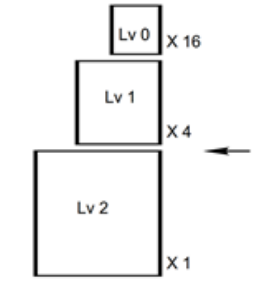

Spatial Pyramid Kernel

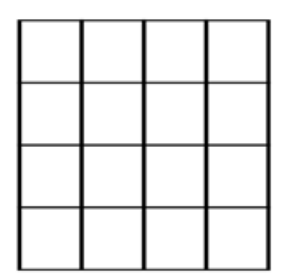

Regular Grid

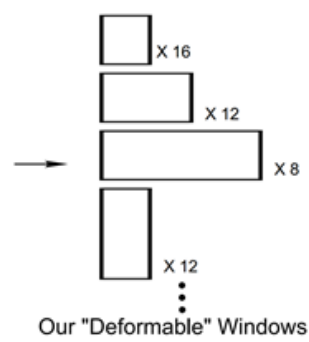

Our "Deformable" Windows (a) The difference between spatial pyramid and flexible partitioning in RAD

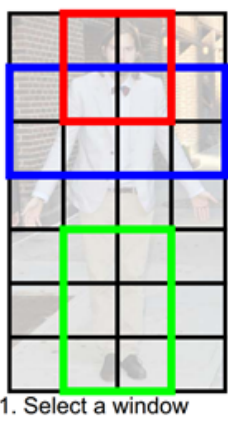

(b) The window specific part learning mechanism in RAD

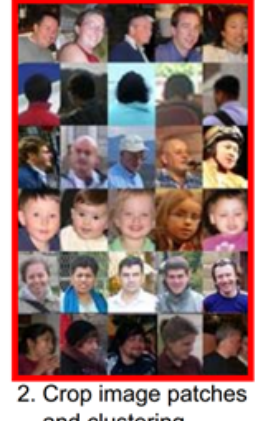

and clustering

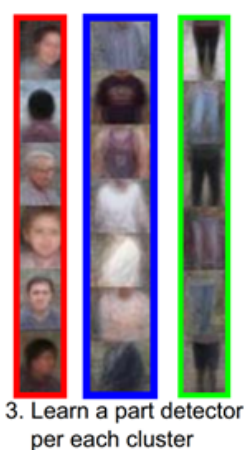

per each cluster the feature to zero. Hence, their model could leverage both the power of convolutional nets for learning discriminative features from data and the ability of poselets to simplify the learning task by decomposing the objects into their canonical poses. They directly feed the combined local and global features into the linear classifier which is a SVM (Support Vector Machine) for multiple attributes estimation.

Fig. 27 (b) illustrate the detailed architecture, this network takes the poselet RGB patch $56 \times 56 \times 3$ as input and output the response score of each attribute with corresponding fully connected layer (fc layer). The feature extraction module contains four groups of convolutional/pooling/normalization layers and the output of these groups are $28 \times 28 \times 64,12 \times 12 \times 64,6 \times 6 \times 64$ and $3 \times 3 \times 64$, respectively. Then the input image is mapped into a feature vector whose dimension is 576-D with a fully connected layer. They set a fc layer for each attribute whose output dimension is 128 .

For the advantage of this work, it adopt deep features rather than shallow low-level features which can obtain more powerful feature representation than previous works. In addition, it also process the human image from the perspective of local patches and global image, which can mining more detailed information than those works who only consider whole image. These two points all improve the person attributes recognition significantly. However, we think the following issues may limit the final performance in their procedure: 1). the parts localization, i.e. the accuracy of poselets, which maybe the bottleneck of their results; 2). they do not use an end-to-end learning framework for the learning of deep features; 3). their poselet also contain background information, which may also influence the feature representation.

\subsubsection{MLCNN (ICB-2015) [11]}

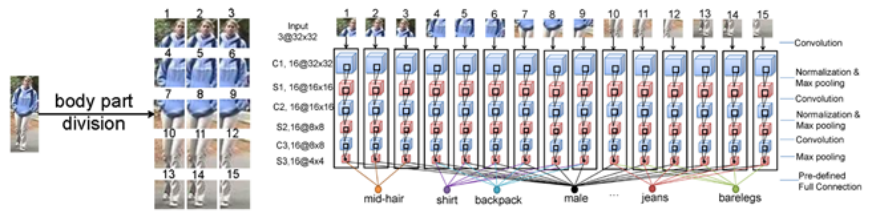

Figure 28. The pipelines of MLCNN [11].

This paper propose a multi-label convolutional neural network to predict multiple attributes together in a unified framework. The overall pipeline of their network can be found in Fig. 28. They divide the whole image into 15 overlapping patches and use a convolutional network to extract its deep features. They adopt corresponding local parts for specific attribute classification, such as the patch 1,2,3 are used for hair style estimation. They utilize softmax function for each attribute prediction.

In addition, they also use the predicted attributes to assist person re-identification. Specifically, they fuse the low-level feature distance and attribute-based distance as final fusion distance to discriminate whether given images have the same identity.

Figure 27. Overview of Pose Aligned Networks for Deep Attribute modeling (PANDA). One convolutional neural net is trained on semantic part patches for each poselet and then the top-level activations of all nets are concatenated to obtain a pose-normalized deep representation. The final attributes are predicted by linear SVM classifier using the posenormalized representations. This figure is adopted from PANDA [10].

As shown in Fig. 27 (a), they first detect the poselets [8] and obtain parts of the person. Then, they adopt the CNN to extract the feature representations of the local patches and whole human image. For the poselet does not detected, they simply leave

\subsubsection{AAWP (ICCV-2015) [12]}

The AAWP is introduced to validate whether parts could bring improvements on both action and attribute recognition. As shown in Fig. 29 (1), the CNN features are computed on a set of bounding boxes associated with the instance to classify, i.e. the whole instance, the oracle or person detector provided and poseletlike part detector provided. The authors define three human body 
parts (head, torso and legs) and cluster the keypoints of each part into several distinct poselets. This part detectors are named deep version of poselets due to the utilization of deep feature pyramid, rather than low-level gradient orientation features used in traditional poselets [8], [91]. In addition, the authors also introduce task-specific CNN fine-tuning and their experiments shown that a fine-tuned holistic model (i.e. no parts) could already achieve comparable performance with part-based system like PANDA [10]. Specifically, the whole pipeline can be divided into two main modules, i.e. the part detector module and fine-grained classification module, as shown in Fig. 29 (2) and (3) respectively.

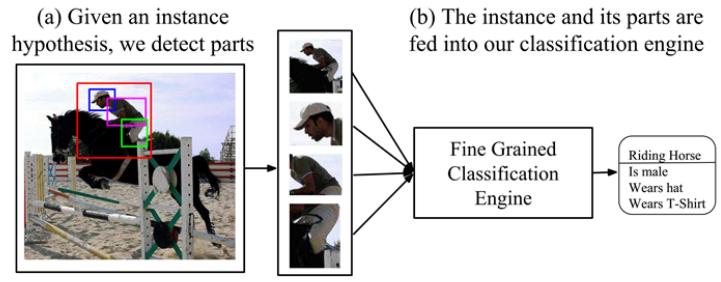

(1). The joint attribute and action recognition based on parts

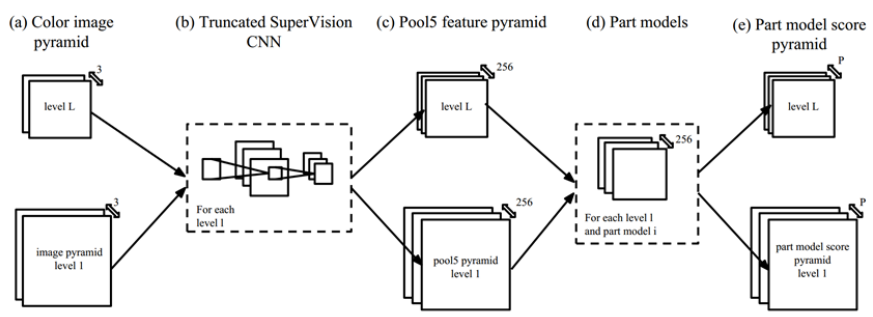

(2). The overview of part detectors

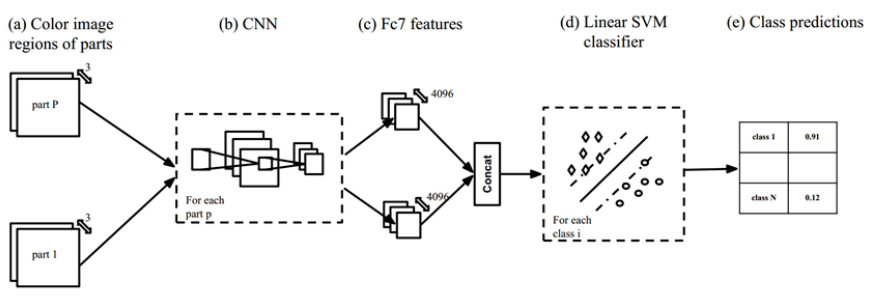

(3). The overview for fine-grained classification using parts

Figure 29. The pipelines of joint action and attribute recognition using parts, part detector and fine-grained classification module, respectively. This figure is adopted from AAWP [12].

For the part detector module, they design their network by following the object detection algorithm RCNN [92] which contain two stages, i.e. the feature extraction and part classification. They adopt a multi-scale fully convolutional network to extract the image features. More specifically, they first construct color image pyramid and obtain the pool5 feature for each pyramid level. Then, they adopt part models to obtain corresponding score, as shown in Fig. 29 (2). Therefore, the key problem lies on how to achieve accurate part localization given these feature map pyramid. To handle the localization of parts, the authors design three body areas (head, torso and legs) and train part detectors with linear SVMs. The positive training data is collected from PASCAL VOC 2012 with cluter algorithm. In the testing phase, they keep the highest scoring part within a candidate region box in an image.

For the task of part-based classification discussed in their paper, i.e. the action and attribute recognition. They consider four different approaches to understand which design factors are important, i.e. no parts, instance fine-tuning, joint fine-tuning and 3way split. The detailed pipeline for the fine-grained classification can be found in Fig. 29 (3). Given the image and detected parts, they use $\mathrm{CNN}$ to obtain fc7 features and concatenated into one feature vector as its final representation. Therefore, the action or attribute category can be estimated with pre-trained linear SVM classifier. Their experiments on the PASCAL VOC action challenge and Berkeley attributes of people dataset [8] validated the effectiveness of part. In addition, they also find that as more powerful convolutional network architectures are engineered, the marginal gain from explicit parts may vanish. They think this might be because of the already high performance achieved by the holistic network.

This work further expand and validate the effectiveness and necessary of parts in a more wider way. It also shown more insights on deep learning based human attributes recognition.

\subsubsection{ARAP (BMVC2016) [13]}

This paper adopt an end-to-end learning framework for joint part localization and multi-label classification for person attribute recognition. As shown in Fig. 30, the ARAP contains following sub-modules: initial convolutional feature extraction layers, a key point localization network, an adaptive bounding box generator for each part, and the final attribute classification network for each part. Their network contain three loss functions, i.e. the regression loss, aspect ratio loss and classification loss.

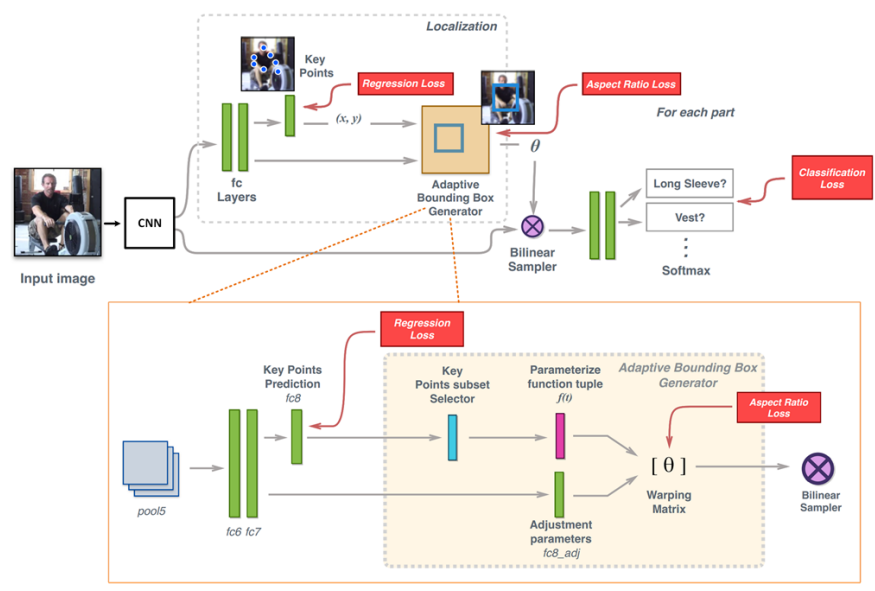

Figure 30. The pipelines of ARAP [13].

Specifically speaking, they first extract the feature map of input image, then conduct key points localization. Given the key points, they divide human body into three main regions (including hard, torso and legs) and obtain an initial part bounding box. On the other hand, they also take previous fc7 layer's features as input and estimate the bounding box adjustment parameters. Given these bounding box, they adopt bilinear sampler to extract corresponding local features. Then, the features are fed into two fc layers for multi-label classification.

\subsubsection{DeepCAMP (CVPR-2016) [14]}

This paper propose a novel $\mathrm{CNN}$ that mines mid-level image patches for fine-grained human attributes recognition. Specifically, they train a $\mathrm{CNN}$ to learn discriminative patch groups, named DeepPattern. They utilize regular contextual information (see Fig. 31 (2)) and also let an iteration of feature learning and patch clustering purify the set of dedicated patches, as shown in Fig. 31 (1). 
The main insight of this paper lies on that a better embedding can help improving the quality of clustering algorithm in pattern mining algorithm [93]. Therefore, they propose an iteration algorithm where in each iteration, they train a new CNN to classify cluster labels obtained in previous iteration to help improving the embedding. On the other hand, they also concatenate features from both local patch and global human bounding box to improve the clusters of mid-level elements.

\subsubsection{PGDM (ICME-2018) [15]}

The PGDM is the first work which attempt to explore the structure knowledge of pedestrian body (i.e. pedestrian pose) for person attributes learning. They first estimate the keypoints of given human image using pre-trained pose estimation model. Then, they extract the part regions according to these keypoints. The deep features of part regions and whole image are all extracted and used for attribute recognition independently. These two scores are then fused together to achieve final attribute recognition. The visualization of pose estimation and the whole pipeline of PGDM can be found in Fig. 32 (a) and (b) respectively.

As shown in Fig. 32 (b), the attribute recognition algorithm contains two main modules: i.e. the main net and PGDM. The main net is a modification of AlexNet [64], the fc8 layer is setting as same with attribute number. It takes the attribute recognition as multi-label classification problem and adopts the improved crossentropy loss [94] as their objective function.

For the PGDM module, its target is to explore the deformable body structure knowledge to assist pedestrian attribute recognition. The authors resort to deep pose estimation models rather than reannotating human pose information of training data. And they embed existing pose estimation algorithm into their attribute recognition model instead of use it as an external one. They directly train a regression network to predict the pedestrian pose with coarse ground truth pose information which obtained from existing pose estimation model ". Once the pose information is obtained, they transform the key points into informative regions using spatial transformer network (STN) [95]. Then, they use independent neural network for feature learning from each key point related region. They jointly optimize the main net, PGDM and pose regression network.

\subsubsection{DHC (ECCV-2016) [16]}

This paper propose to use deep hierarchical contexts to help person attribute recognition due to the background would sometimes provide more information than target object only. Specifically, the human-centric context and scene context is introduced in their network architecture. As shown in Fig. 33, they first construct input image pyramid and pass them all through CNN (VGG-16 network is used in this paper) to obtain multi-scale feature maps. They extract features of four set of bounding box regions, i.e. the whole person, detected parts of target object, nearest neighbor parts from the image pyramid and global image scene. The first two branches (the whole person and parts) are regular pipeline for person attributes recognition algorithm. The main contributions of this paper lies on the later two branches, i.e. the human-centric and scene-level contexts to help improving the recognition results. Once the scores of these four branches are obtained, they sum up all the scores as final attribute score.

I The human key points list used in PGDM are: head, neck, right shoulder, right elbow, right wrist, left shoulder, left elbow, left wrist, right hip, right knee, right ankle, left hip, left knee, left ankle.
Due to the use of context information, this neural network need more external training data than regular pedestrian attribute recognition task. For example, they need to detect the part of human body (head, upper and bottom body regions) and recognize the style/scene of given image. They propose a new dataset named WIDER, to better validate their ideas. Although the human attribute recognition results can be improved significantly via this pipeline, however, this model looks a little complex than other algorithms.

\subsubsection{LGNet (BMVC-2018) [17]}

This paper propose a Localization Guide Network (named LGNet) which could localize the areas corresponding to different attributes. It also follows the local-global framework, as shown in Fig. 34. Specifically, they adopt Inception-v2 [96] as their basic CNN model for feature extraction. For the global branch, they adopt global average pooling layer (GAP) to obtain its global features. Then, a fully connected layer is utilized to output its attribute predictions. For the local branch, they use $1 \times 1$ convolution layer to produces $c$ class activation maps for each image, where $c$ is the number of attributes in the used dataset. Given the class activation maps, they can capture an activation box for each attribute by cropping the high-response areas of the corresponding activation map. They also use EdgeBoxes [97] to generate regions proposals to obtain local features from the input image. In addition, they also consider the different contributions of extracted proposals and different attributes should focus on different local features. Therefore, they use the class active map for each attribute to serve as a guide to determine the importance of the local features to different attributes. More specifically, they compute the spatial affinity map between the proposals and class activation boxes according to the Interaction over Union (IoU) and linearly normalized to weight the local feature vectors for further predictions. Finally, the global and attended local features are fused together by element-wise sum for pedestrian attribute prediction.

Summary: On the basis of the reviewed papers [8] [9] [10] [11] [12] [13] [14] [15] [16] [17], it is easy to find that these algorithms all adopt the joint utilization of global and fine-grained local features. The localization of body parts is achieved via an external part localization module, such as part detection, pose estimation, poselets or proposal generation algorithm. The use of part information improve the overall recognition performance significantly. At the same time, it also bring some shortcomings as follows: Firstly, as an operation in the middle phase, the final recognition performance is heavily relays on the accuracy of part localization. In another word, the inaccurate part detection results will bring the wrong features for final classification. Secondly, it will also need more training or inference time due to the introduce of human body parts. Thirdly, some algorithms need manual annotated labels about part locations which further increase the cost of manpower and money.

\subsection{Attention-based Models}

In this section, we will talk about person attribute recognition algorithms using attention mechanism, such as HydraPlus-Net [18], VeSPA [19], DIAA [20], CAM [21].

\subsubsection{HydraPlus-Net (ICCV-2017) [18]}

HPNet is introduced to encode multi-scale features from multiple levels for pedestrian analysis using multi-directional attention 


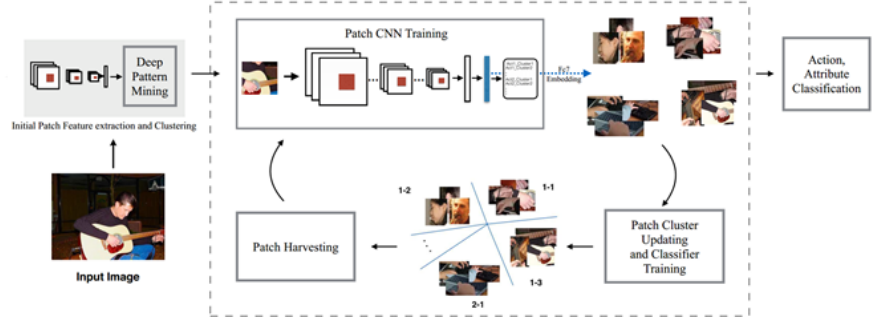

(1) The pipeline for training mid-level deep visual elements in action and attribute

Figure 31. The overview of DeepCAMP [14].



(a) Visualization of the leamed regions for each key point

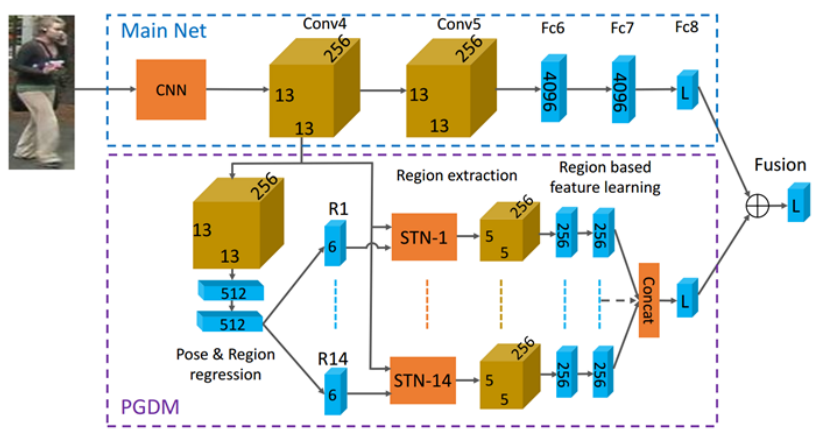

(b) The overall framework of PGDM

Figure 32. The visualization of pose estimation and pipeline of PGDM. This figure is adopted from PGDM [15].

(MDA) modules. As shown in Fig. 35 (2), it contains two main modules i.e. the Main Net (M-net) which is a regular $\mathrm{CNN}$ and the Attentive Feature Net (AF-net) which includes multiple branches of multi-directional attention modules applied to different semantic feature levels. The AF-net and M-net share same basic convolution architectures and their outputs are concatenated and fused by global average pooling (GAP) and fc layers. The output layer can be the attribute logits for attribute recognition or feature vectors for person re-identification. The authors adopt inceptionv2 [98] as their basic network.

A specific illustration of AF-net can be found in Fig. 35 (4). Given the feature maps of black 1, 2 and 3, they conduct $1 \times 1$ convolution operation on feature map 2 and obtain its attention map $\alpha^{2}$. It is worthy to note that, this attention module is different from previous attention based models which only push the attention map back to the same block. They not only use this attention map to attend feature map 2, but also attend the adjacent features, such as feature map 1 and 3. Applying one single attention map to multiple blocks naturally let the fused features encode multi-level information within the same spatial distribution, as shown in Fig. 35 (3).

The HP-net is trained in a stage-wise manner, in another word, the M-net, AF-net and remaining GAP and fc layers are trained in a sequential way. The output layer is used to minimize the crossentropy loss and softmax loss for person attribute recognition and person re-identification respectively.

\subsubsection{VeSPA (ArXiv-2017) [19]}

The VeSPA takes the view cues into consideration to better estimate corresponding attribute. Because the authors find that the visual cues hinting at attributes can be strongly localized and inference of person attributes such as hair, backpack, shorts, etc., are highly dependent on the acquired view of the pedestrian. As shown in Fig. 36, the image is feed into the Inceptions ( $\mathrm{K}$ layers) and obtain its feature representation. The view-specific unit is introduced to mapping the feature maps into coarse attribute prediction $\hat{y}_{a t t}=\left[y^{1}, y^{2}, \ldots, y^{c}\right]^{T}$. Then, a view predictor is used to estimate the view weights $\hat{y}_{\text {view }}$. The attention weights is used to multiply view-specific predictions and obtain the final multiclass attribute prediction $\hat{Y}_{c}=\left[y^{1}, y^{2}, \ldots, y^{C}\right]^{T}$.

The view classifier and attribute predictors are trained with separate loss function. And the whole network is a unified framework and can be trained in an end-to-end manner.

\subsubsection{DIAA (ECCV-2018) [20]}

The DIAA algorithm can be seen as an ensemble method for person attribute recognition. As shown in Fig. 37, their model contain following modules: multi-scale visual attention and weighted focal loss for deep imbalanced classification. For the multi-scale visual attention, as we can see from Fig. 37, the authors adopt feature maps from different layers. They propose the weighted focal loss function [99] to measure the difference between predicted attribute vectors and ground truth:

$$
\begin{array}{r}
\mathcal{L}_{w}\left(\hat{y}_{p}, y\right)=-\sum_{c=1}^{C} w_{c}\left(\left(1-\sigma\left(\hat{y}_{p}^{c}\right)\right)^{\gamma} \log \left(\sigma\left(\hat{y}_{p}^{c}\right)\right) y^{c}+\right. \\
\left.\sigma\left(\hat{y}_{p}^{c}\right)^{\gamma} \log \left(1-\sigma\left(\hat{y}_{p}^{c}\right)\right)\left(1-y^{c}\right)\right),
\end{array}
$$

where $\gamma$ is a parameter which is used to control the instance-level weighting based on the current prediction giving emphasis to the hard misclassified samples. $w_{c}=e^{-a_{c}}$ and $a_{c}$ is the prior class distribution of $c^{t h}$ attribute following [19].

In addition, they also propose to learn the attention maps in a weakly supervised manner (only the attribute labels, no specific bounding box annotation) to improve the classification 


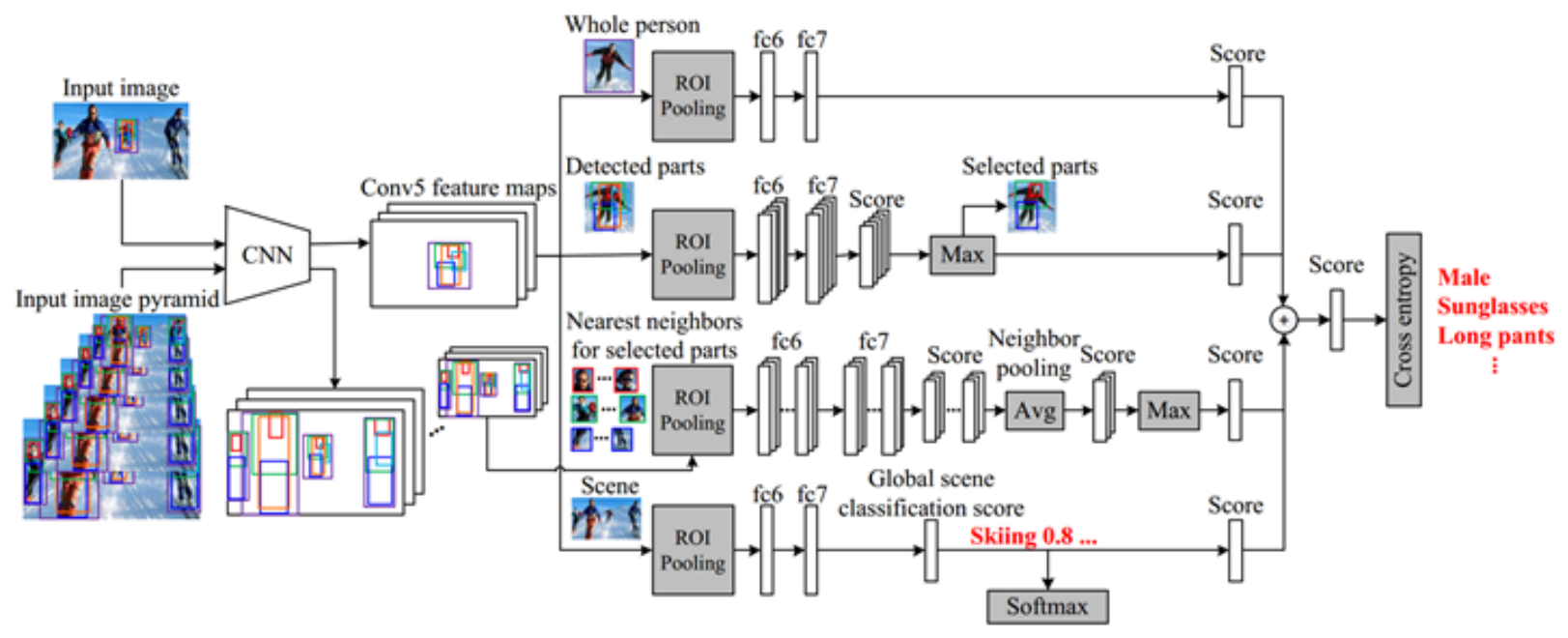

Figure 33. The pipeline of DHC-network. This figure is adopted from DHC [16].

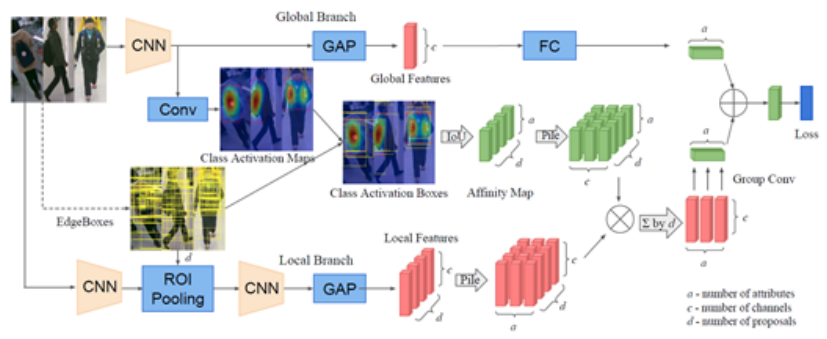

Figure 34. The visualization of pose estimation and pipeline of LGNet. This figure is adopted from LGNet [17].

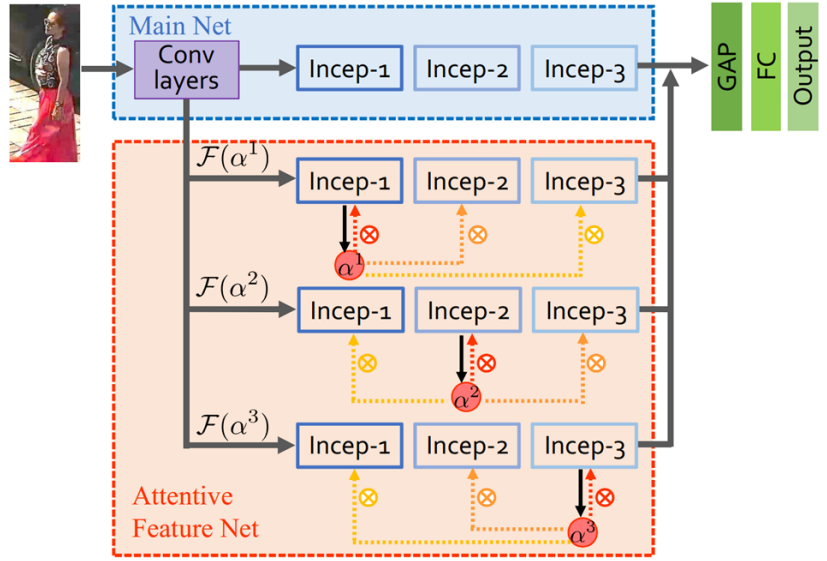

Figure 35. Some visualizations of HydraPlus-Net. This figure is adopted from HydraPlus-Net [18].

performance by guiding the network to focus its resources to those spatial parts that contain information relevant to the input image. As shown in the right part of Fig. 37, the attention subnetwork takes the feature map $W_{i} \times H_{i} \times F_{i}$ as input and output an attention mask with dimension $W_{i} \times H_{i} \times C$. The output is then feed to attention classifier to estimate the pedestrian attributes. Due to limited supervised information for the training of attention module, the authors resort to the prediction variance by following [100]. Attention mask predictions with high standard deviation

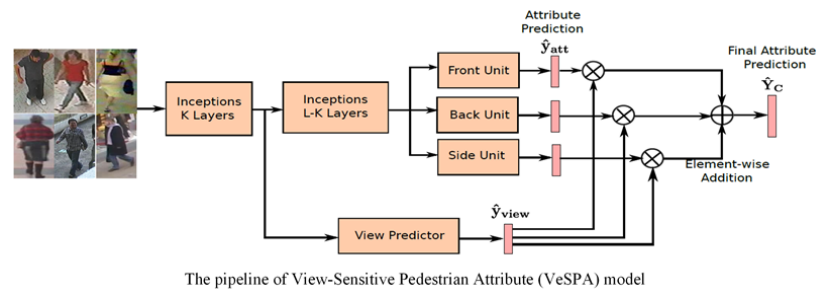

Figure 36. The pipeline of VeSPA model. This figure is adopted from VeSPA model [19].

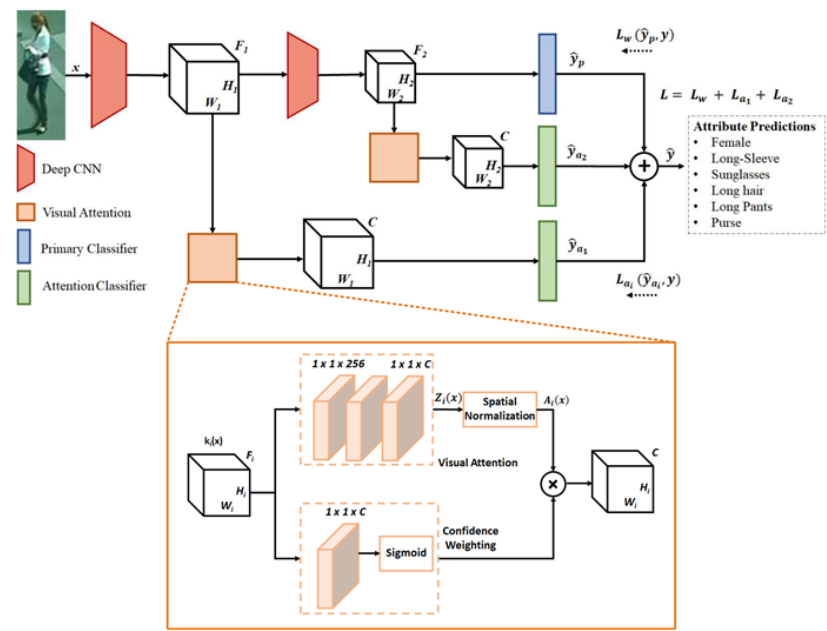

Figure 37. The pipeline of DIAA model. This figure is adopted from DIAA model [20].

across time will be given higher weights in order to guide the network to learn those uncertain samples. They collect the history $H$ of the predictions for the $s^{t h}$ sample and compute the standard deviation across time for each sample in a mini-batch. Hence, the loss for the attention map with attribute-level supervision for each sample $s$ can be obtained by:

$$
\mathcal{L}_{a_{i}}\left(\hat{y}_{a_{i}}, y\right)=\left(1+\operatorname{std}_{s}(H)\right) \mathcal{L}_{b}\left(\hat{y}_{a_{i}}, y\right)
$$

where $\mathcal{L}_{b}\left(\hat{y}_{a_{i}}, y\right)$ is binary cross entropy loss and $\operatorname{std}_{s}(H)$ is the 
standard deviation.

Therefore, the total loss used to train this network end-to-end is the sum of loss from the primary network and the two attention modules.

\subsubsection{CAM (PRL-2017) [21]}

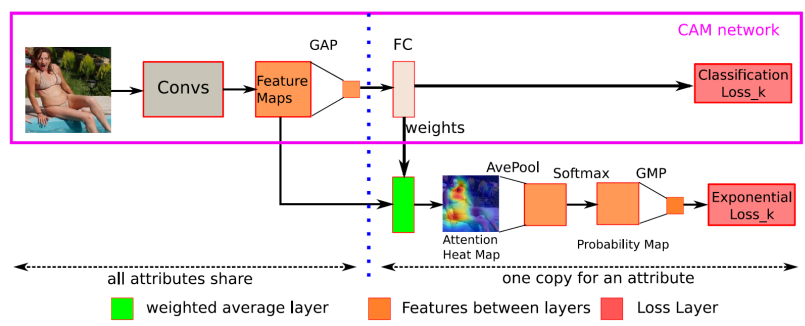

Figure 38. The pipeline of CAM-network [21].

In this paper, the authors propose to use and refine attention map to improve the performance of person attribute recognition. As shown in Fig. 38, their model contain two main modules, i.e. the multi-label classification sub-network and attention map refinement module. The adopted CAM net [101] also follows the category-specific framework, in another word, different attribute classifier have different parameters for the fully connected (FC) layer. They use the parameters in FC layer as weights to linearly combine the feature maps in the last convolutional layer to obtain the attention map of each object category. However, this naive implementation of attention mechanism could not focus on the right regions all the time due to low resolution, over-fitting training, et al.

To handle aforementioned issues, they explore to refine the attention map by tuning CAM network. They measure the appropriateness of an attention map based on its concentration and attempt to make the attention map to highlight a smaller but concentrated region. Specifically speaking, they introduce a weighted average layer to obtain attention map first. Then, they use average pooling to down-sample its resolution to capture the importance of all the potential relevant regions. After that, they also adopt Softmax layer to transform the attention map into a probability map. Finally, the maximum probability can be obtained via the global average pooling layer.

On the basis of the maximum probability, the authors propose a new loss function (named exponential loss function) to measure the appropriateness of the attention heat map which can be written as:

$$
L=\frac{1}{N} e^{\alpha\left(P_{i j}^{M}+\beta \mu\right)}
$$

where $P_{i j}^{M}$ is the maximum probability for image $i$ and attribute $j . \alpha$ and $\beta$ are hyper-parameters and $\mu=1 / H^{2}$ is the mean value of the probability map. $H \times H$ is the size of attention (and probability) map. For the training of the network, the authors first pre-training the CAM network only by minimizing classification loss; then, they adopt joint loss functions to fine-tuning the whole network.

Summary: Visual attention mechanism has been introduced in pedestrian attribute recognition, but the existing works are still limited. How to design new attention models or directly borrow from other domains is still needed to be explored in this area.

\subsection{Sequential Prediction based Models}

In this section, we will give a review of Sequential Prediction based models for person attribute recognition including $\mathrm{CNN}$ RNN [22], JRL [23], GRL [24], JCM [25] and RCRA [102].

\subsubsection{CNN-RNN (CVPR-2016) [22]}

Regular multi-label image classification framework learn independent classifier for each category and employ ranking or threshold on the classification results, fail to explicitly exploit the label dependencies in an image. This paper first adopts RNNs to address this problem and combine with CNNs to learn a joint imagelabel embedding to characterize the semantic label dependency as well as the image-label relevance. As shown in Fig. 39, the red and blue dots are the label and image embeddings, respectively. The image and recurrent neural output embeddings are summed and denoted with black dots. This mechanism could model the label co-occurrence dependencies in the joint embedding space by sequentially linking the label embeddings. It can compute the probability of a label based on the image embedding $I$ and output of recurrent neurons $x_{t}$, which can be formulated as:

$$
s(t)=U_{l}^{T} x_{t}
$$

where $x_{t}=h\left(U_{o}^{x} o(t)+U_{I}^{x} I\right), U_{o}^{x}$ and $U_{I}^{x}$ are the projection matrices for recurrent layer of output and image representation, respectively. $U_{l}$ is the label embedding matrix. $o(t)$ is the outputs of the recurrent layer at the time step t.

For the inference of CNN-RNN model, they attempt to find the sequence of labels that maximize the priori probability:

$$
\begin{array}{r}
l_{1}, \ldots, l_{k}=\arg \max _{l_{1}, \ldots, l_{k}} P\left(l_{1}, \ldots, l_{k} \mid I\right) \\
=\arg \max _{l_{1}, \ldots, l_{k}} P\left(l_{1} \mid I\right) \times P\left(l_{2} \mid I, l_{1}\right) \ldots P\left(l_{k} \mid I, l_{1}, \ldots, l_{k-1}\right)
\end{array}
$$

They adopt beam search algorithm [103] for the top-ranked prediction path as their estimation result. The training of the $\mathrm{CNN}$ RNN model can be achieved by cross-entropy loss function and back-propagation through time (BPTT) algorithm.

The CNN-RNN model is very similar with deep models used in image caption task [104] [105]. They all take one image as input and output a series of words under the encoder-decoder framework. The main difference is that the caption model output one sentence and the CNN-RNN model generates attributes (but these attributes are also related with each other). Therefore, we can borrow some techniques from image caption community to help improve the performance of pedestrian attribute recognition.

\subsubsection{JRL (ICCV-2017) [23]}

This paper first analyse the existing learning issues in the pedestrian attribute recognition task, e.g. poor image quality, appearance variation and little annotated data. They propose to explore the interdependency and correlation among attributes and visual context as extra information source to assist attribute recognition. Hence, the JRL model is proposed to joint recurrent learning of attribute context and correlation, as its name shows. The overall pipeline of JRL can be found in Fig. 40.

To better mining these extra information for accurate person attribute recognition, the authors adopt sequence-to-sequence model to handle aforementioned issues. They first divide the given person image $I$ into $m$ horizontal strip regions and form a region sequences $S=\left(s_{1}, s_{2}, \ldots, s_{m}\right)$ in top-bottom order. The obtained region sequences $S$ can be seen as the input sentence in natural 

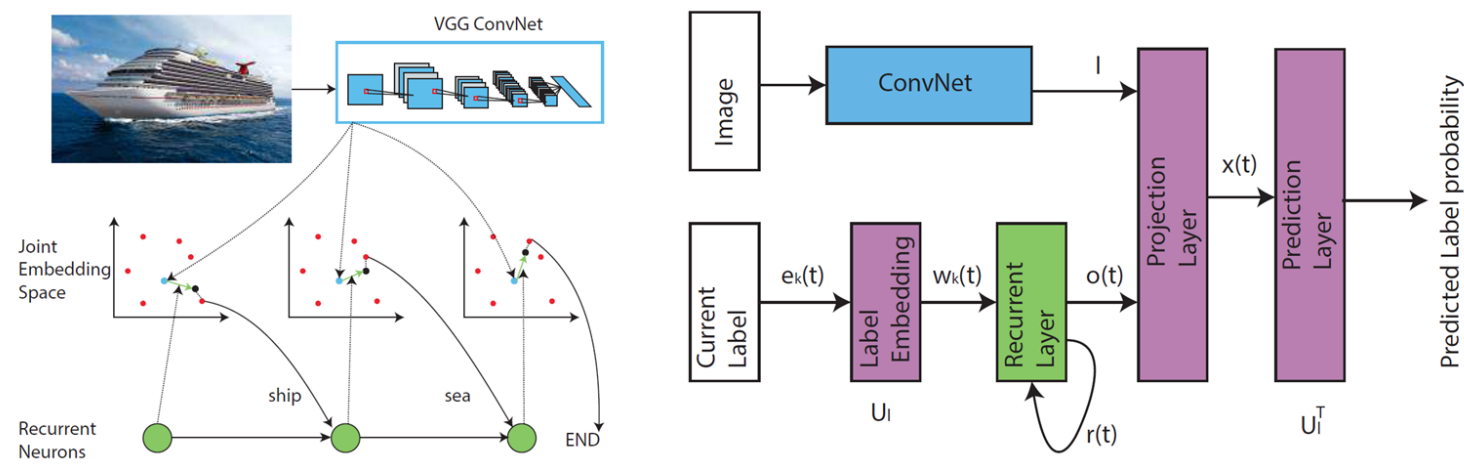

Figure 39. The illustration and architecture of the proposed CNN-RNN model for multi-label classification [22].

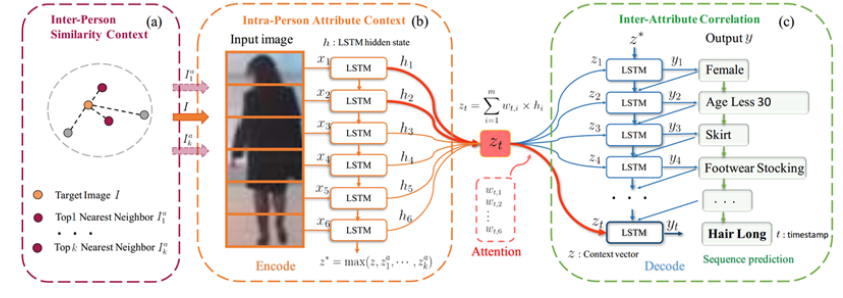

Figure 40. The pipeline of Joint Recurrent Learning (JRL) of attribute context and correlation. This figure is adopted from JRL [23].

language processing, and can be encoded with the LSTM network in a sequential manner. The hidden state $h^{e n}$ of the encoder LSTM can be updated based on regular LSTM update procedure, as shown in Eq. 6. The final hidden state $h_{m}^{e n}$ can be seen as the summary representation $z=h_{m}^{e n}$ of the whole person image (named as context vector). This feature extraction procedure could model the intra-person attribute context within each person image $I$.

To mining more auxiliary information to handle the appearance ambiguity and poor image quality in a target image. The authors resort to the visually similar exemplar training images and introduce these samples to model the inter-person similarity context constraint. They first search top- $k$ samples that are similar with target image with CNN features based on L2 distance metric, and compute its own context vector $z_{i}^{a}$. Then, all the context vector representations are ensembled as the inter-person context $z^{*}$ with max-pooling operation.

In the decoding phase, the decoder LSTM takes both intraperson attribute context $(z)$ and inter-person similarity context $\left(z^{*}\right)$ as input and output variable-length attributes over time steps. The attribute prediction in this paper can also be seen as a generation scheme. To better focus on local regions of person image for specific attributes and obtain more accurate representation, they also introduce the attention mechanism to attend the intra-person attribute context. For the final attribute estimation order, they adopt the ensemble idea to incorporate the complementary benefits of different orders and thus capture more high-order correlation between attributes in context.

\subsubsection{GRL (IJCAI-2018) [24]}

GRL is developed based on JRL which also adopt the RNN model to predict the human attributes in a sequential manner. Different from JRL, GRL is formulated to recognize human attributes by group step by step to pay attention to both intra-group and intergroup relationships. As shown in Fig. 41 (1), the author divide the whole attribute list into many groups because the attributes in intra-group are mutual exclusive and have relations between intergroup. For example, BoldHair and BlackHair cannot occur on the same person image, but they are both related to the head-shoulder region of a person and can be in the same group to be recognized together. It is an end-to-end single model algorithm with no need for preprocessing and it also exploit more latent intra-group and inter-group dependency among grouped pedestrian attributes. The overall algorithm can be found in Fig. 41 (2).

As shown in Fig. 41 (2), given the human image, they first detect the key points and locate the head, upper body and lower body regions using body region generation module. They extract the whole image features with Inception-v3 network and obtain the local part features using ROI average pooling operation. It is worthy to note that all attributes in the same group share the same fully connected feature. Given the global and local features, they adopt LSTM to model the spatial and semantic correlations in attribute groups. The output of each LSTM unit is then feeded into fully connected layer and a prediction vector can be obtained. This vector has the same dimension as the number of attributes in the relevant group. They also use a batch normalize layer to balance the positive and negative outputs of this network.

\subsubsection{JCM (arXiv-2018) [25]}

Existing sequential prediction based person attribute recognition algorithms, such as JRL [23], GRL [24], may be easily influenced by different manual division and attributes orders due to the weak alignment ability of RNN. This paper propose a joint CTCAttention model (JCM) to conduct attribute recognition, which could predict multiple attribute value with arbitrary length at a time avoiding the influence of attribute order in the mapping table.

As shown in Fig. 42, the JCM is actually a multi-task network which contains two tasks, i.e. the attribute recognition and person re-identification. They use ResNet-50 as the basic model to extract features for both tasks. For the attribute recognition, they adopt the Transformer [106] as their attention model for the alignment of long attribute sequence. And the connectionist temporal classification (CTC) loss [107] and cross entropy loss functions are used for the training of network. For the person re-ID, they directly use two fully connected layers (i.e. the dense model) obtain feature vectors and use softmax loss function to optimize this branch.

In the test phase, the JCM could simultaneously predicts the person identity and a set of attributes. They also use beam search 


\begin{tabular}{|l|c|}
\hline Group & Attribute \\
\hline Gender & male or female \\
\hline Age & age 16-30, age 31-45, age 46-60, age 60+ \\
\hline Head & hair length, muffler, hat, glasses \\
\hline Upper Body & clothes style, logo, casual or formal \\
\hline Lower Body & clothes style, casual or formal \\
\hline Footware & footware style \\
\hline Accessories & backpack, messenger bag, plastic bag etc \\
\hline
\end{tabular}

(1) The groups of 35 binary attributes in PETA dataset

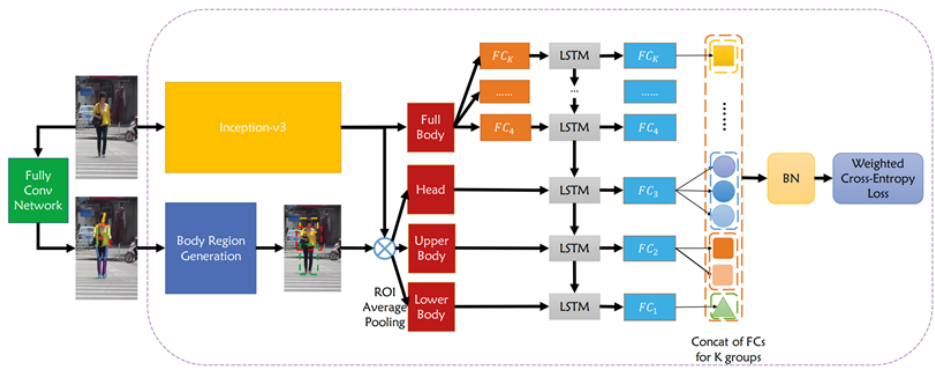

(2) The pipeline of Grouping Recurrent Learning architecture

Figure 41. The pipeline of Group Recurrent Learning (GRL). This figure is adopted from GRL [24].

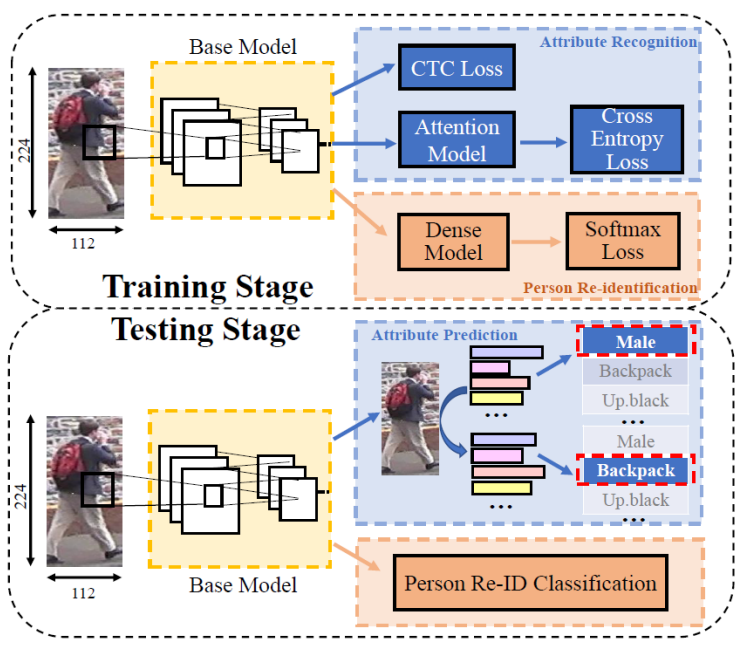

Figure 42. The overview of JCM which is proposed in [25].

for the decoding of attribute sequence. Meanwhile, they extracts the features from the CNN in base model to classify pedestrians for person re-ID task.

\subsubsection{RCRA (AAAl-2019) [102]}

This paper propose two models, i.e., Recurrent Convolutional (RC) and Recurrent Attention (RA) for pedestrian attributes recognition, as shown in Figure 43. The RC model is used to explore the correlations between different attribute groups with Convolutional-LSTM model [108] and the RA model takes the advantage of the intra-group spatial locality and inter-group attention correlation to improve the final performance.

Specifically speaking, they first divide all the attributes into multiple attribute groups, similar with GRL [24]. For each pedestrian image, they use CNN to extract its feature map and feed to ConvLSTM layer group by group. Then, new feature maps for each time step can be obtained by adding a convolutional network after ConvLSTM. Finally, the features is used for attribute classification on current attribute group.

Based on aforementioned RC model, they also introduce visual attention module to highlight the region of interest on the feature map. Given the image feature map $F$ and the heat map $H_{t}$ of attention at each time step $t$, the attended feature map $F_{t}$ for current attribute group can be obtained via:

$$
F_{t}=\operatorname{sigmoid}\left(H_{t}\right) \otimes F+F
$$

where $\otimes$ denotes the spatial point-wise multiplication. The attended feature maps is used for final classification. The training of this network is also based on weighted cross-entropy loss function proposed in WPAL-network [26].



Figure 43. The overview of RCRA which is proposed in [102].

Summary: As we can see from this subsection, these algorithms all adopt the sequential estimation procedure. Because the attributes are correlated to each other, and they also have various difficulty. Therefore, it is an interesting and intuitive idea to adopt the RNN model to estimate the attributes one by one. Among these algorithms, they integrate different neural networks, attribute groups, multi-task learning into this framework. Compared with CNN based methods, these algorithms are more elegant and effective. The disadvantage of these algorithms is the time efficiency due to the successive attribute estimation. In the future works, more efficient algorithms for the sequential attributes estimation are needed.

\subsection{Loss Function based Models}

In this section, we will review some algorithms with improved loss function, including WPAL [26], AWMT [27].

\subsubsection{WPAL-network (BMVC-2017) [26]}

The WPAL is proposed to simultaneously recognize and locate the person attributes in a weakly-supervised manner (i.e. only person attribute labels, no specific bounding box annotation). As shown in Fig. 44, the GoogLeNet is adopted as their basic network for feature extraction. They fuse features from different layers (i.e. the features from Conv $3-E, C o n v 2-E$ and $C o n v 1-E$ layers) and feed into Flexible Spatial Pyramid Pooling layer (FSPP). Compared with regular global max-pooling, the advantage of FSPP can be listed as following two aspects: 1). it can add spatial constraint to some attributes like hats; 2). the structure lays in the 


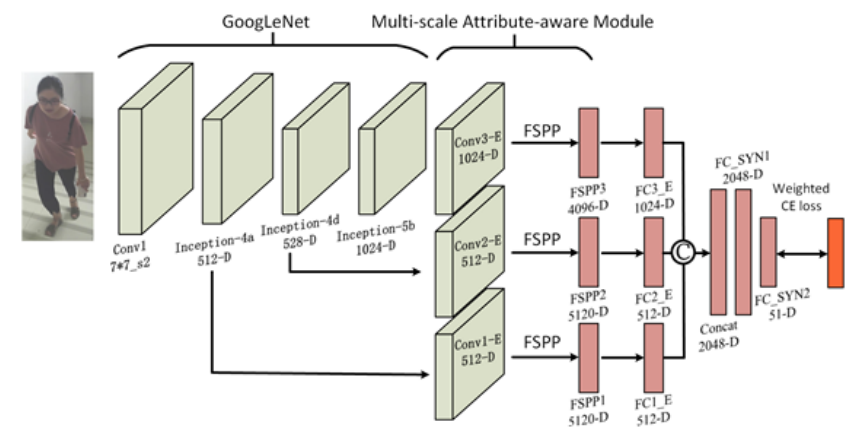

Figure 44. The pipeline of WPAL-network. This figure is rewritten based on WPAL-network [26].

middle stage of the network but not the top, making correlation between detector and target class not bound at first but free to be learnt during training. The outputs of each FSPP are feed into fully connected layers and output a vector whose dimension is same with the number of pedestrian attributes.

In the training procedure, the network could simultaneously learning to fit the following two targets: the first one is learn the correlation between attributes and randomly initialized midlevel detectors, and the second one is to adapt the target mid-level features of detectors to fit the correlated attributes. The learnt correlation, the detection results of mid-level features are later used to locate the person attributes.

In addition, the authors also introduce a novel weighted cross entropy loss function to handle the extremely imbalanced distribution of positive and negative samples of most attribute categories. The mathematical formulation can be written as follows:

Loss $_{w c e}=\sum_{i=1}^{L} \frac{1}{2 w_{i}} * p_{i} * \log \left(\hat{p}_{i}\right)+\frac{1}{2\left(1-w_{i}\right)}\left(1-p_{i}\right) * \log \left(1-\hat{p}_{i}\right)$

where $L$ denote the number of attributes, $p$ is the ground truth attribute vector, $\hat{p}$ is the estimated attribute vector, $w$ is a weight vector indicating the proportion of positive labels over all attribute categories in the training dataset.

\subsubsection{AWMT (MM-2017) [27]}

As is known to all, the learning difficulty of various attributes is different. However, most of existing algorithms ignore this situation and share relevant information in their multi-task learning framework. This will leads to negative transfer, in another word, the inadequate brute-force transfer may hurt the learners performance when two tasks are dissimilar. AWMT propose to investigate an sharing mechanism that is possible of dynamically and adaptively coordinating the relationships of learning different person attribute tasks. Specifically, they propose an adaptively weighted multi-task deep framework to jointly learn multiple person attributes, and a validation loss trend algorithm to automatically update the weights of weighted loss layer. The pipeline of their network can be found in Fig. 45.

As shown in Fig. 45, they adopts ResNet-50 as basic network and takes both train and val images as input. The basic network will output its predicted attribute vectors for both train and val images. Hence, the train loss and val loss can be obtained simultaneously. The val loss is used to update the weight vectors $\lambda_{j}(j=1, \ldots, M)$ which is then utilized to weight different

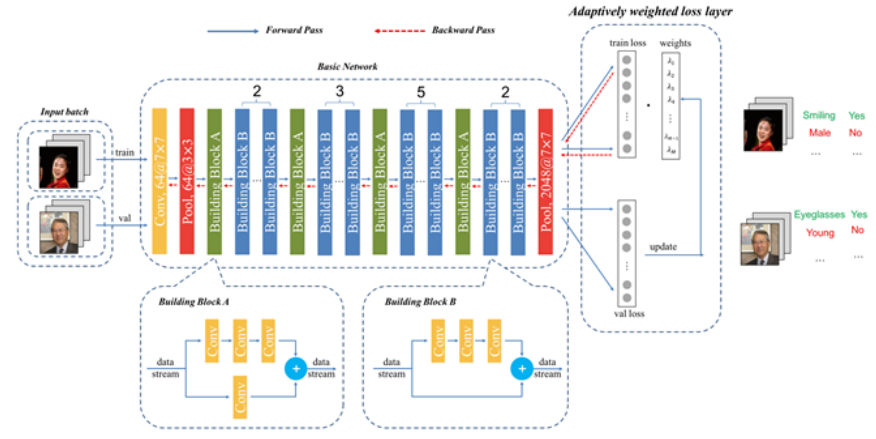

Figure 45. The pipeline of AWMT-network. This figure is adopted from AWMT [27].

attributes learning. The adaptive weighted loss function can be formulated as follows:

$$
\Theta=\arg \min _{\Theta} \sum_{j=1}^{M} \sum_{i=1}^{N}<\lambda_{j}, \mathcal{L}\left(\psi_{j}\left(\mathbf{I}_{i} ; \Theta\right)-\mathbf{L}_{i j}\right)>
$$

where $\Theta$ denote the parameters of neural network, $\lambda_{j}$ is the scale value to weight the importance of the task of learning $j$ th attributes. $\mathbf{I}_{i}$ denote the $i$-th image in a mini-batch, $\mathbf{L}_{i j}$ is the ground truth label of attribute $j$ of image $i . \psi_{j}\left(\mathbf{I}_{i} ; \Theta\right)$ is the predicted attributes of input image $\mathbf{I}_{i}$ under neural network parameter $\Theta .<\cdot\rangle$ is the inner product operation.

The key problem is how to adaptively tuning the weight vector $\lambda_{j}$ in Eq. 24. They propose the validate loss trend algorithm to realize this target. The intuition behind their algorithm is that in learning multiple tasks simultaneously, the "important" tasks should be given high weight (i.e. $\lambda_{j}$ ) to increase the scale of loss of the corresponding tasks. But the question is how we can know which task is more "important", in another word, how do we measure the importance of one task?

In this paper, the authors propose to use the generalization ability as an objective measurement. Specifically, they think the trained model of one task with lower generation ability should be set higher weight than those models of the other tasks. The weight vector $\lambda_{j}$ is updated per $k$ iterations and used to compute the loss of training data and update the network parameter $\Theta$ in the backward pass. Their experiments on several attributes dataset validated the effectiveness of this adaptive weighting mechanism.

Summary: There are few works focus on design new loss functions for pedestrian attribute recognition. WPAL-network [26] consider the unbalanced distribution of data and propose a weighted cross-entropy loss function according to the proportion of positive labels over all attribute categories in the training dataset. This methods seems a little tricky but has been widely used in many PAR algorithms. AWMT [27] propose an adaptive weighting mechanism for each attribute learning to make the network focus more on handle the "hard" tasks. These works full demonstrate the necessary to design novel loss functions to better train the PAR network.

\subsection{Curriculum Learning based Algorithms}

In this subsection, we will introduce the curriculum learning based algorithm which consider to learning the human attribute in a "easy" to "hard" way, such as: MTCT [109], CILICIA [110]. 

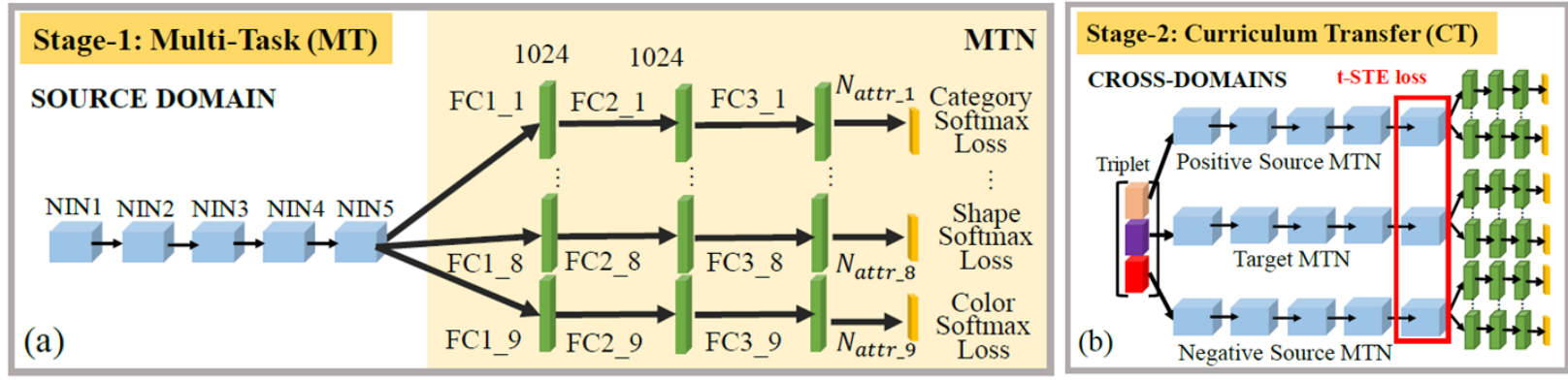

Figure 46. The MTCT network design [109].

\subsubsection{MTCT (WACV-2017) [109]}

This paper propose a multi-task curriculum transfer network to handle the issue on the lack of manually labelled training data. As shown in Fig. 46, their algorithm mainly contain multi-task network and curriculum transfer learning.

For the multi-task network, they adopt five stacked NetworkIn-Network (NIN) convolutional units [111] and $N$ parallel branches, with each branch representing a three layers of fully connected sub-network for modelling one of the $N$ attributes respectively. The Softmax loss function is adopted for the model training.

Inspired by cognitive studies that suggest a better learning strategy adopted by human and animals is to start with learning easier tasks before gradually increasing the difficulties of the tasks, rather than to blindly learn randomly organised tasks. Therefore, they adopt curriculum transfer learning strategy for clothing attribute modelling. Specifically, it is consisted of two main stages. In the first stage, they use the clean (i.e. easier) source images and their attribute labels to train the model. In the second stage, they embedding cross-domain image pair information and simultaneously appending harder target images into the model training process to capture harder cross-domain knowledge. They adopt tSTE (t-distribution stochastic triplet embedding) loss function to train the network which can be described as:

$$
\begin{gathered}
L_{t-S T E}=\sum_{I_{t}, I_{p s}, I_{n s} \in T} \\
\log \frac{\left(1+\frac{\left\|f_{t}\left(I_{t}\right)-f_{s}\left(I_{p s}\right)\right\|^{2}}{\alpha}\right)^{\beta}}{\left(1+\frac{\left\|f_{t}\left(I_{t}\right)-f_{s}\left(I_{p s}\right)\right\|^{2}}{\alpha}\right)^{\beta}+\left(1+\frac{\left\|f_{t}\left(I_{t}\right)-f_{s}\left(I_{n s}\right)\right\|^{2}}{\alpha}\right)^{\beta}}
\end{gathered}
$$

where $\beta=-0.5 *(1+\alpha)$ and $\alpha$ is the freedom degree of the Student kernel. $f_{t}(\cdot)$ and $f_{s}(\cdot)$ are the feature extraction function for target and source multi-task network, respectively.

\subsubsection{CILICIA (ICCV-2017) [110]}

Similar with MTCT [109], CILICIA [110] also introduce the idea of curriculum learning into person attribute recognition task to learning the attributes from easy to hard. The pipeline of CILICIA can be found in Fig. 47. They explore the correlations between different attribute learning tasks and divide such correlations into strongly and weakly correlated tasks. Specifically, under the framework of multi-task learning, they use the respective Pearson correlation coefficients to measure the strongly correlated tasks which can be formulated as:

$$
p_{i}=\sum_{j=1, j \neq i}^{T} \frac{\operatorname{cov}\left(y_{t_{i}}, y_{t_{j}}\right)}{\sigma\left(y_{t_{i}} \sigma\left(y_{t_{j}}\right)\right)}, i=1, \ldots, T
$$

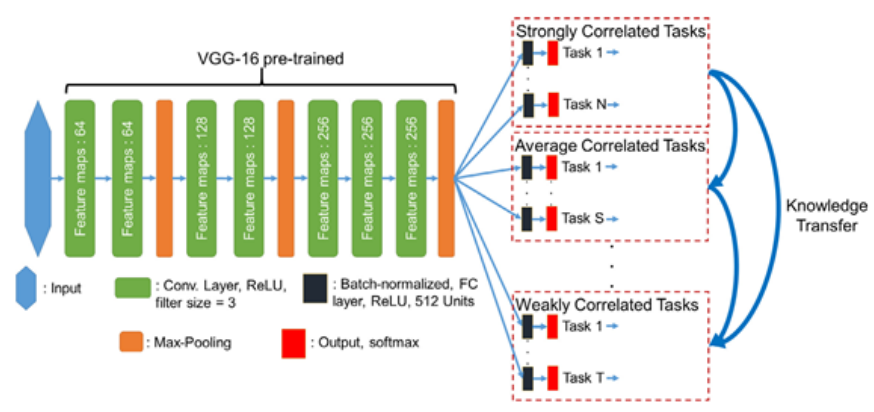

Figure 47. The CILICIA network designed in [112] .

where $\sigma\left(y_{t_{i}}\right)$ is the standard deviation of the labels $y$ of the task $t_{i}$. The tasks of $p_{i}$ with top $50 \%$ are strongly correlated with rest and can be divided into strongly correlated group. And the rest tasks are belong to weakly correlated group and will learning under the guidance of knowledge learned from strongly correlated group.

For the multi-task network, they adopt the categorical crossentropy function [113] between predictions and targets, which can be defined as follows (for a single attribute $\mathrm{t}$ ):

$$
L_{t}=\frac{1}{N} \sum_{i=1}^{N} \sum_{j=1}^{M}\left(\frac{1 / M_{j}}{\sum_{n=1}^{M} 1 / M_{n}}\right) \cdot \mathbb{1}\left[y_{i}=j\right] \cdot \log \left(p_{i, j}\right)
$$

where $\mathbb{1}\left[y_{i}=j\right]$ is one if the target of sample $i$ belong to class $j$, and zero otherwise. $M_{j}$ is the number of samples belonging to class $j, \mathrm{M}$ and $\mathrm{N}$ are the number of classes and samples, respectively.

To weight different attribute learning tasks, one intuitive idea is to learn another branch network for weights learning. However, the authors did not see significant improvement with this method. Therefore, they adopt the supervision transfer learning technique [114] to help attribute learning in weakly correlated group:

$$
L_{w}=\lambda \cdot L_{s}+(1-\lambda) \cdot L_{w}^{f},
$$

where $L_{w}^{f}$ is the total loss obtained during the forward pass using Eq. 27 only over the weakly correlated tasks.

They also propose CILICIA-v2 [112] by proposing an effective method to obtain the groups of tasks using hierarchical agglomerative clustering. It can be of any number and not just only two groups (i.e. strong/weakly correlated). More specifically, they employ the computed Pearson correlation coefficient matrix to perform hierarchical agglomerative clustering using the Ward variance minimization algorithm. Wards method is biased towards generating clusters of the same size and analyses all possible pairs 
of joined clusters, identifying which joint produces the smallest within cluster sum of squared (WCSS) errors. Therefore, we can obtain attribute groups via the WCSS threshold operation. For each group, they compute learning sequence of clusters by sorting the obtained respective Pearson correlation coefficients only within the clusters. Once the total dependencies for all the clusters formed, the curriculum learning process can be started in a descending order.

Summary: Inspired by recent progress of cognitive science, the researchers also consider to use such "easy" to "hard" learning mechanism for PAR. They introduce existing curriculum learning algorithm into their learning procedure to model the relations between each attribute. Some other algorithms such as self-paced learning [115] is also used to model the multi-label classification problem [116] or other computer vision tasks [117]. It is also worthy to introduce more advanced works of cognitive science to guide the learning of PAR.

\subsection{Graphic Model based Algorithms}

Graphic models are commonly used to model structure learning in many applications. Similarly, there are also some works to integrate these models into the pedestrian attribute recognition task, for example: DCSA [28], A-AOG [29], VSGR [30].

\subsubsection{DCSA (ECCV-2012) [28]}

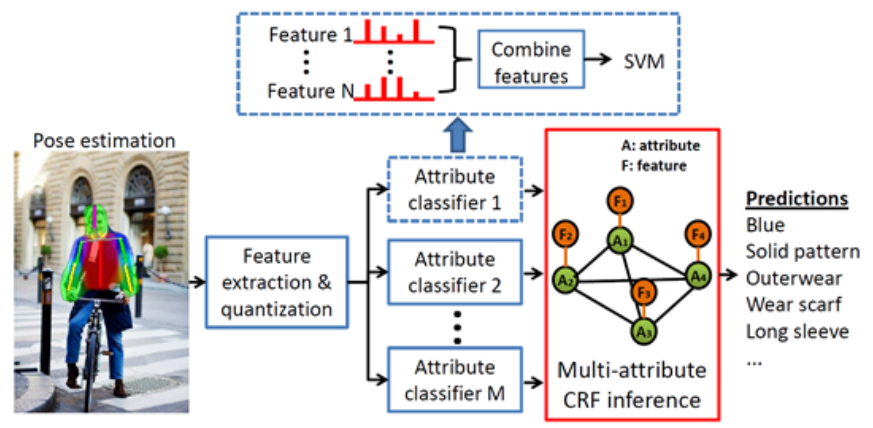

Figure 48. The pipelines of DCSA [28].

In this paper, the authors propose to model the correlations between human attributes using conditional random field (CRF). As shown in Fig. 48, they first estimate the pose information using off-the-shelf algorithms [118] and locate the local parts of upper body only (the lower-body is ignored because of the occlusion issues). Then, four types of base features are extracted from these regions, including SIFT [2], texture descriptor [119], color in LAB space, and skin probabilities. These features are fused to train multiple attribute classifiers via SVM. The key idea of this paper is apply the fully connected CRF to explore the mutual dependencies between attributes. They treat each attribute functions as a node of CRF and the edge connecting every two attribute nodes reflects the joint probability of these two attributes. The belief propagation [120] is adopted to optimize the attribute label cost.

\subsubsection{A-AOG (TPAMI-2018) [29]}

The A-AOG model which is short for attribute And-Or grammar, is proposed explicitly represents the decomposition and articulation of body parts, and account for the correlations between poses and attributes. This algorithm is developed based on And-Or graph [121] and the and-nodes denote decomposition or dependency; the

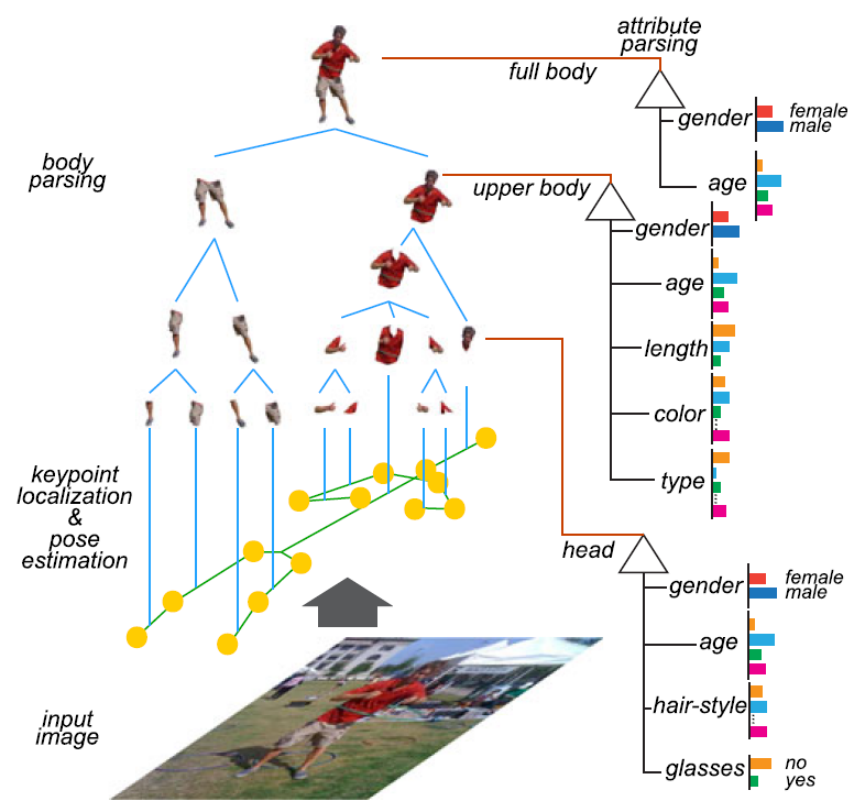

Figure 49. An attributed parse graph for a human image [29].

or-nodes represent alternative choices of decomposition or types of parts. Specifically speaking, it mainly integrate the three types of grammars: phrase structure grammar, dependency grammar and an attribute grammar.

Formally, the A-AOG is defined as a five-tuple:

$$
A-A O G=<S, V, E, X, \mathcal{P}>
$$

where $V$ is the vertex set and it mainly contains set of and-nodes, or-nodes and terminal nodes: $V=V_{\text {and }} \cup V_{\text {or }} \cup V_{T} ; E$ is the edge set and it consists of two subsets $E=E_{p s g} \cup E_{d g}$ : set of edges with phrase structure grammar $E_{p s g}$ and dependency grammar $E_{d g} . X=\left\{x_{1}, x_{2}, \ldots, x_{N}\right\}$ is the attribute set associated with nodes in $V . \mathcal{P}$ is the probability model on graphical representation.

According to aforementioned definitions, the parse graph can be formulated as:

$$
p g=(V(p g), E(p g), X(p g))
$$

An example of the parse graph derived from the A-AOG can be found in Fig. 50. Given an image $I$, the target is to find the most probable parse graph $\mathrm{pg}$ from their grammar model. They adopt the Bayesian framework which computes the joint posterior as the product of a likelihood and prior probability, to formulate the probability model over the parse graph as follows:

$$
\begin{aligned}
& P(p g \mid I ; \lambda) \propto P(I \mid p g ; \lambda) P(p g ; \lambda) \\
= & \frac{1}{Z} \exp \{-\mathcal{E}(I \mid p g ; \lambda)-\mathcal{E}(p g ; \lambda)\}
\end{aligned}
$$

where $\lambda$ is the model parameters. The energy functions $\mathcal{E}$ can be decomposed into a set of potential functions. The both terms in Eq. 31 can be decomposed into part and attribute relations, therefore, the Eq. 31 can be rewritten as:

$$
\begin{array}{r}
P(p g \mid I ; \lambda)=\frac{1}{Z} \exp \left\{-\mathcal{E}_{a p p}^{V}(I \mid p g ; \lambda)-\mathcal{E}_{a p p}^{X}(I \mid p g ; \lambda)\right. \\
\left.-\mathcal{E}_{r e l}^{V}(p g ; \lambda)-\mathcal{E}_{r e l}^{X}(p g ; \lambda)\right\}
\end{array}
$$

where $\mathcal{E}_{\text {app }}^{V}(I \mid p g ; \lambda), \mathcal{E}_{\text {app }}^{X}(I \mid p g ; \lambda), \mathcal{E}_{\text {rel }}^{V}(p g ; \lambda)$ and $\mathcal{E}_{\text {rel }}^{X}(p g ; \lambda)$ 


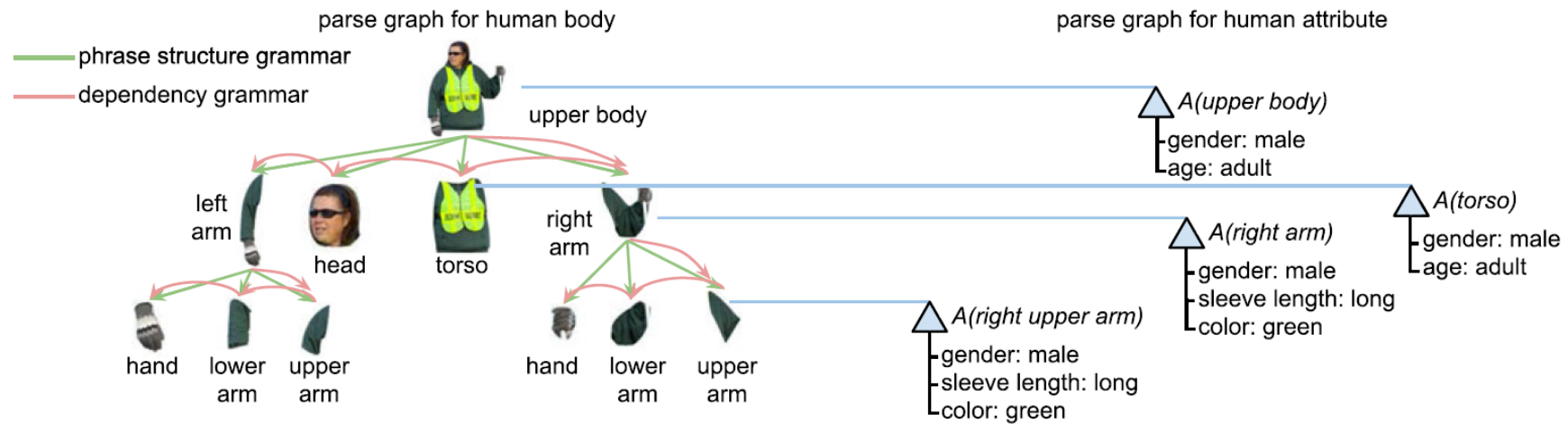

Figure 50. A parse graph example derived from the A-AOG [29], which contains parse graphs for human body detection and pose and human attributes.

are appearance and relations terms for part and attribute respectively.

Then, the energy terms can be expressed as following scoring functions:

$$
\begin{aligned}
S(p g \mid I) & =-\mathcal{E}_{a p p}^{V}(I \mid p g)-\mathcal{E}_{a p p}^{X}(I \mid p g)-\mathcal{E}_{\text {rel }}^{V}(p g)-\mathcal{E}_{\text {rel }}^{X}(p g) \\
& =S_{a p p}^{V}(I, p g)+S_{a p p}^{X}(I, p g)+S_{r e l}^{V}(p g)+S_{r e l}^{X}(p g) .
\end{aligned}
$$

Therefore, the most probable parse graph $p g^{*}$ can be found by maximizing the score function 33 :

$$
\begin{array}{r}
p g^{*}=\arg \max _{p g} P(I \mid p g) P(p g) \\
=\arg \max _{p g}\left[S_{a p p}^{V}(p g, I)+S_{a p p}^{X}(p g, I)+S_{r e l}^{V}(p g)+S_{r e l}^{X}(p g)\right] \\
\approx \arg \max _{p g}\left[S_{a p p}(p g, I)+S_{r e l}(p g)\right]
\end{array}
$$

They use deep CNN to generate the proposals for each part and adopt greedy algorithm based on the beam search to optimize aforementioned objective function. For the detailed learning and inference procedure, please check the original paper [122] [29].

\subsubsection{VSGR (AAAI-2019) [30]}

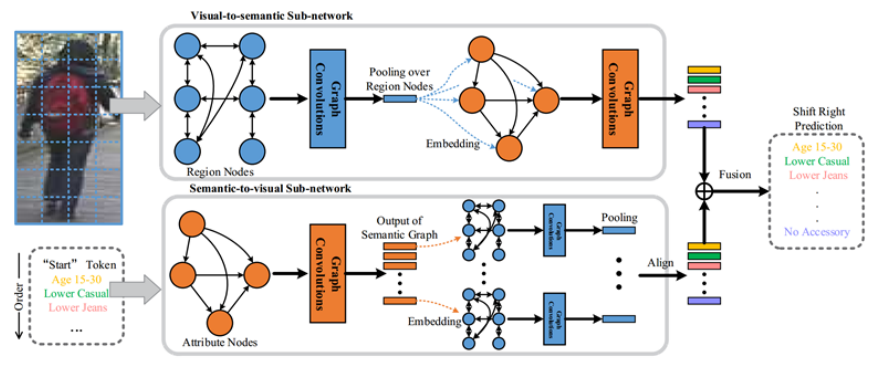

Figure 51. The overview of visual-semantic graph reasoning framework (VSGR) which is proposed in [30].

In this paper, the authors propose to estimate the pedestrian attributes via visual-semantic graph reasoning (VSGR). They argue that the accuracy of person attribute recognition is heavy influenced by: 1). only local parts are related with some attributes; 2). challenging factors, such as pose variation, viewpoint and occlusion; 3). the complex relations between attributes and different part regions. Therefore, they propose to jointly model spatial and semantic relations of region-region, attribute-attribute, and regionattribute with a graph-based reasoning framework. The overall pipeline of their algorithm can be found in Fig. 51.
As shown in Fig. 51, this algorithm mainly contain two subnetworks, i.e. the visual-to-semantic sub-network and semantic-tovisual sub-network. For the first module, it first divide the human image into a fixed number of local parts $\mathbf{X}=\left(x_{1}, x_{2}, \ldots, x_{M}\right)^{T}$. They construct a graph whose node is the local part and edge is the similarity of different parts. Different from regular relation modelling, they adopt both the similarity relations between parts and topological structures to connect one part with its neighbour regions. The similarity adjacency matrix can be formulated as:

$$
\mathbf{A}_{s_{a}}(i, j)=\frac{\exp \left(\mathbf{F}_{s}\left(x_{i}, x_{j}\right)\right)}{\sum_{j=1}^{M} \exp \left(\mathbf{F}_{s}\left(x_{i}, x_{j}\right)\right)}
$$

where $\mathbf{F}_{s}\left(x_{i}, x_{j}\right)$ denote the pairwise similarity between each two part regions which can also be modelled by neural network.

And the topological relations between local parts can be obtained via:

$$
\mathbf{A}_{s_{l}}(i, j)=\frac{\exp \left(-d_{i j} / \Delta\right)}{\sum_{j=1}^{M} \exp \left(-d_{i j} / \Delta\right)}
$$

where $d_{i j}$ is the pixel distance between two parts and $\Delta$ is the scaling factor.

The two sub-graphs is combined to compute the output of spatial graph via the following equation:

$$
\mathbf{G}_{s}=\mathbf{A}_{s_{a}} \mathbf{X} \mathbf{W}_{s_{a}}+\mathbf{A}_{s_{l}} \mathbf{X} \mathbf{W}_{s_{l}}
$$

where $\mathbf{W}_{s_{a}}$ and $\mathbf{W}_{s_{l}}$ are weight matrices for two sub-graphs.

Therefore, the spatial context representation $g_{s}$ can be obtained via average pooling operation after convolution. After encode the region-to-region relation, they also adopt similar operation to model the relations between semantic attributes based on spatial context. The node of new graph is the attributes, and they transform them into the embedding matrix $\mathbf{R}=\left(r_{0}, r_{1}, \ldots, r_{K}\right)$, where $r_{0}$ denotes the "start" token and each column $r_{i}$ is an embedding vector. The positional encoding [78] is also considered to make use of the attribute order information $\mathbf{P}=\left(p_{0}, p_{1}, \ldots, p_{K}\right)$. The embedding matrix and positional encoding are combined together to obtain the semantic representations on an ordered prediction path $\mathbf{E}=\left(e_{0}, e_{1}, \ldots, e_{K}\right)$, where $e_{k}=r_{k}+p_{k}$.

Finally, the spatial and semantic context can be obtained by:

$$
\mathbf{C}=\mathbf{E}+\left(\mathbf{U}_{s} g_{s}\right)
$$

where the $\mathbf{U}$ is learnable projection matrix. For the edges, they only connect the $i$-th node with nodes whose subscript $\leq i$ to 
ensure the prediction of current attribute only has relations with previously known outputs. The edge weights of connected edges can be computed by:

$$
\mathbf{F}_{\hat{e}\left(\mathbf{c}_{i}, \mathbf{c}_{j}\right)}=\phi_{\hat{e}}\left(\mathbf{c}_{i}\right)^{T} \phi_{\hat{e}^{\prime}}\left(\mathbf{c}_{j}\right)
$$

where $\phi_{\hat{e}}(*)$ and $\phi_{\hat{e}^{\prime}}(*)$ are linear transformation functions. The adjacency matrix $\mathbf{A}_{\hat{e}}$ can also be obtained by normalizing the connected edge weights along each row. And the convolution operation on semantic graph can be computed as:

$$
\mathbf{G}_{\hat{e}}=\mathbf{A}_{\hat{e}} \mathbf{C}^{T} \mathbf{W}_{\hat{e}}
$$

The output representation $\mathbf{G}_{\hat{e}}$ can be obtained after conduct convolutions on semantic graph, and then utilized for sequential attribute prediction.

The semantic-to-visual sub-network can also be processed in similar manner and it also output sequential attribute prediction. The output of these two sub-networks are fused as the final prediction and can be trained in an end-to-end way.

Summary: Due to the relations existed in multiple attributes, many algorithms are proposed to mine such information for PAR. Therefore, the Graphic models are the first to think and introduced into the learning pipeline, such as Markov Random Field [123], Conditional Random Field [124], And-Or-Graph [121] or Graph Neural Networks [125]. The works reviewed in this subsection are the outputs via integrate the Graphic models with PAR. Maybe the other Graphic models can also be used for PAR to achieve better recognition performance. Although these algorithms have so many advantages, however, these algorithms seems more complex than others. The efficiency issues is also need to be considered in practical scenarios.

\subsection{Other Algorithms}

This subsections is used to demonstrate algorithms that not suitable for aforementioned categories, including: PatchIt [31], FaFS [32], GAM [33].

\subsubsection{Patchlt (BMVC-2016) [31]}

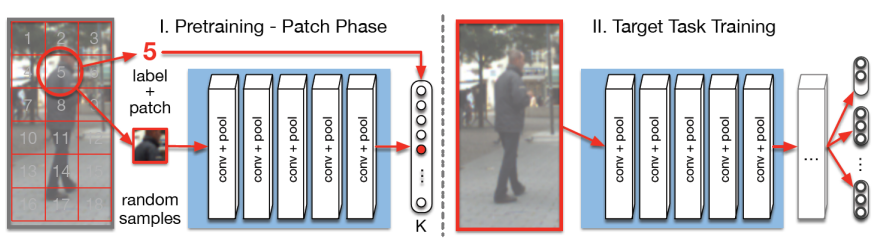

Figure 52. The pre-training algorithm introduced from Patchlt [31] (left figure) and multi-class classification network for PAR (right figure).

Regular ConvNets usually adopt pre-trained model on an auxiliary task for weight initialization. However, it constrain the designed network to as similar as existing architectures, such as AlexNet, VGG or ResNet. Different from these algorithms, this paper propose a self-supervised pre-training approach, named PatchTask, to obtain weight initializations for the PAR. It's key insight is to leverage data from the same domain as the target task for pre-training and the it only relies on automatically generated rather than human annotated labels. In addition, it is easier for us to find massive unlabelled data for our task.

For the PatchTask, the authors define it as a K-class classification problem. As shown in Fig. 52, they first divide the image into multiple non-overlapping local patches, then, let the network to predict the origin of given patch. They use the PatchTask to obtain an initialization for the convolutional layers of VGG16 and applied for PAR.

\subsubsection{FaFS (CVPR-2017) [32]}

The target of multi-task learning is to share relevant information across these tasks to help improve final generalization performance. Most hand-designed deep neural networks conduct both shared and task-specific feature learning. Different from existing works, FaFS [32] is proposed to design compact multi-task deep learning architecture automatically. This algorithm starts with a thin multi-layer network and dynamically widens it in a greedy manner during training. This will create a tree-like deep architecture by repeating above widening procedure and similar tasks reside in the same branch until at the top layer. Fig. 53 (right sub-figure) illustrate this process. Fig. 53 (left figure) gives a comparison between the thin network and VGG-16 model. The weight parameters of thin network is initialized by minimizing the objective function as follows with simultaneous orthogonal matching pursuit (SOMP) [126]:

$$
A^{*}, \omega^{*}(l)=\arg \min _{A \in \mathbb{R}^{d \times d^{\prime}},|w|=d^{\prime}}\left\|W^{p, l}-A W_{w:}^{p, l}\right\|_{F},
$$

where $W^{p, l}$ is the parameters of the pre-trained model at layer $l$ with $d$ rows. $W_{w:}^{p, l}$ denote a truncated weight matrix at only keeps the rows indexed by the set $\omega$. This initialization process is done layer by layer and applicable for both convolutional and fully connected layers.

Then, a layer-wise model widening is adopted to widen the thin network, as shown in Fig. 53 (right sub-figure). This operation is started from output layer and recursively in a top-down manner towards the lower layers. It is also to worthy to note that each branch is associated with a sub-set of tasks. They also separate the similar and dissimilar tasks into different groups according to the probability which is a affinity between a pair of tasks.

\subsubsection{GAM (AVSS-2017) [33]}

This paper propose to handle the issue of occlusion and low resolution of pedestrian attributes using deep generative models. Specifically, their overall algorithm contains three sub-networks, i.e., the attribute classification network, the reconstruction network and super-resolution network.

For the attribute classification network, they also adopt joint global and local parts for final attribute estimation, as shown in Fig. 54. They adopt ResNet50 to extract the deep features and global-average pooling to obtain corresponding score. These scores are fused as the final attribute prediction score. To handle the occlusion and low-resolution problem, they introduce the deep generative adversarial network [87] to generate re-constructed and super-resolution images. And use the pre-processed images as input to the multi-label classification network for attribute recognition.

\section{Applications}

Visual attributes can be seen as a kind of mid-level feature representation which may provide important information for high-level human related tasks, such as person re-identification [128], [129], [130], [131], [132], pedestrian detection [133], person tracking [134], person retrieval [135], [136], human action recognition 

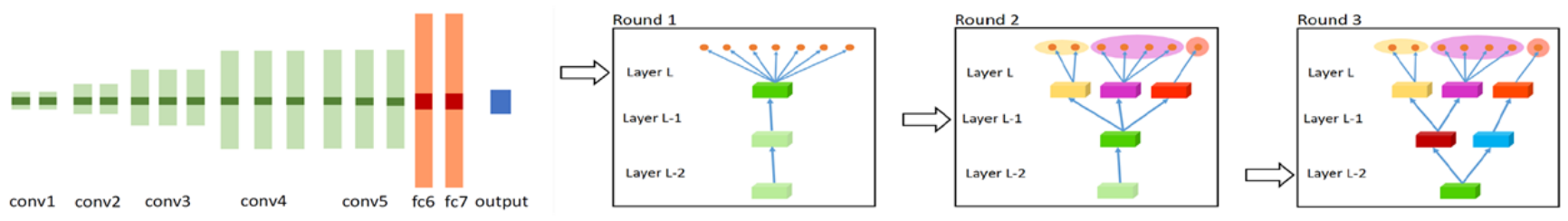

Figure 53. (Left) the comparison between the thin model with VGG-16; (Right) Illustration of the widening procedure in [32]

Table 2

A summary of the source code

\begin{tabular}{c|c}
\hline \hline Algorithm & Source Code \\
\hline DeepMAR [6] & https://github.com/dangweili/pedestrian-attribute-recognition-pytorch \\
Wang et al. [127] & https://github.com/James-Yip/AttentionImageClass \\
Zhang et al. [36] & https://github.com/dangweili/RAP \\
PatchIt [31] & https://github.com/psudowe/patchit \\
PANDA [10] & https://github.com/facebookarchive/pose-aligned-deep-networks \\
HydraPlus-Net [18] & https://github.com/xh-liu/HydraPlus-Net \\
WPAL-Net [26] & https://github.com/YangZhou1994/WPAL-network \\
DIAA [20] & https://github.com/cvcode18/imbalanced_learning \\
\hline
\end{tabular}

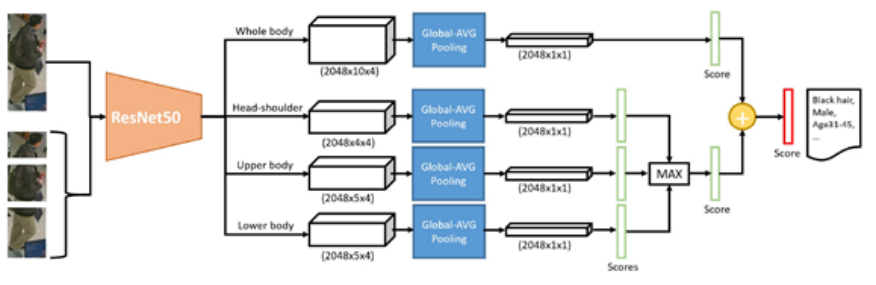

Figure 54. The pipeline of GAM-network. This figure is adopted from GAM [33].

[137], scene understanding [138]. Due to the limited space of this paper, we only review some works in the rest of this subsections.

Pedestrian Detection. Different from regular person detection algorithms which treat it as a single binary classification task, Tian et al. propose to jointly optimize person detection with semantic tasks to address the confuse of positive and hard negative samples. They use existing scene segmentation dataset to transfer attribute information to learn high-level features from multiple tasks and dataset sources. Their overall pipeline can be found in Figure 55.

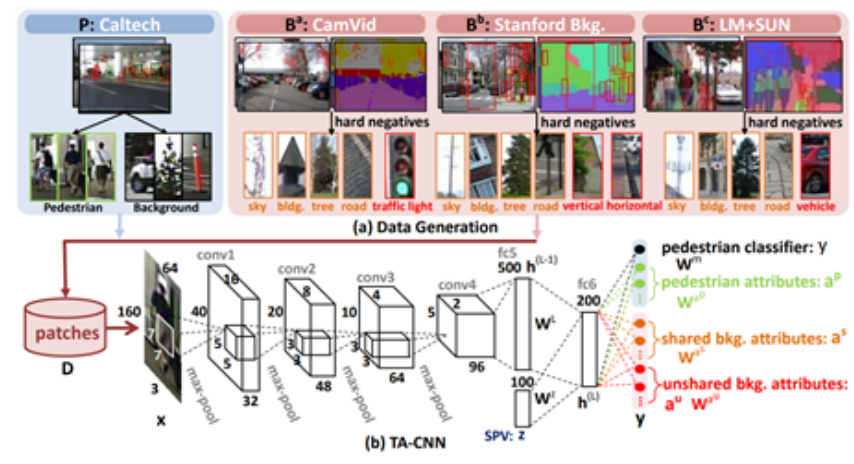

Figure 55. The pipeline of pedestrian detection aided by deep learning semantic tasks [133].

Person Re-identification. As noted in [132], person re- identification and attribute recognition share a common target at the pedestrian description. PAR focuses on local information mine while person re-identification usually capture the global representations of a person. As shown in Figure 56, Lin et al. propose the multi-task network to estimate the person attributes and person ID simultaneously. Their experiments validated the effectiveness of more discriminative representation learning.

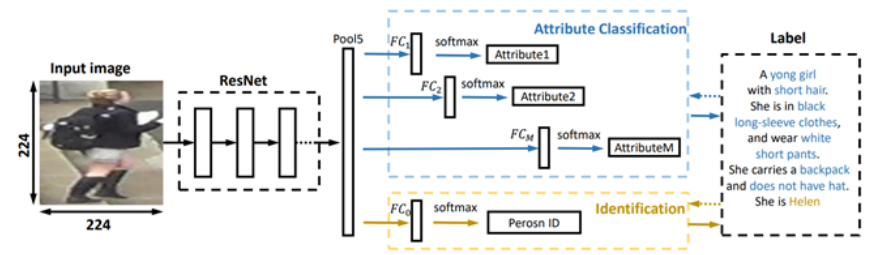

Figure 56. The pipeline of APR network in [132].

$\mathrm{Su}$ et al. also propose to integrate the mid-level human attributes into person re-identification framework in [128]. They train the attribute model in semi-supervised manner and mainly contain three stages, as shown in Figure 57. They first pre-train the deep CNN on an independent attribute dataset, then, fine-tuned on another dataset only annotated with person IDs. After that, they estimate attribute labels for target dataset using the updated deep CNN model. They can achieve good results on multiple person re-ID datasets using the deep attributes with simple Cosine distance. Sameh Khamis et al. [130] propose to integrate a semantic aspect into regular appearance-based methods. They jointly learn a discriminative projection to a joint appearance-attribute subspace, which could effectively leverage the interaction between attributes and appearance for matching. Li et al. also present a comprehensive study on clothing attributes assisted person re-ID in [131]. They first extract the body parts and their local features to alleviate the pose-misalignment issues. Then, they propose a latent SVM based person re-ID approach to model the relations between low-level part features, middle-level clothing attributes and high-level re-ID labels of person pairs. They treat the clothing 


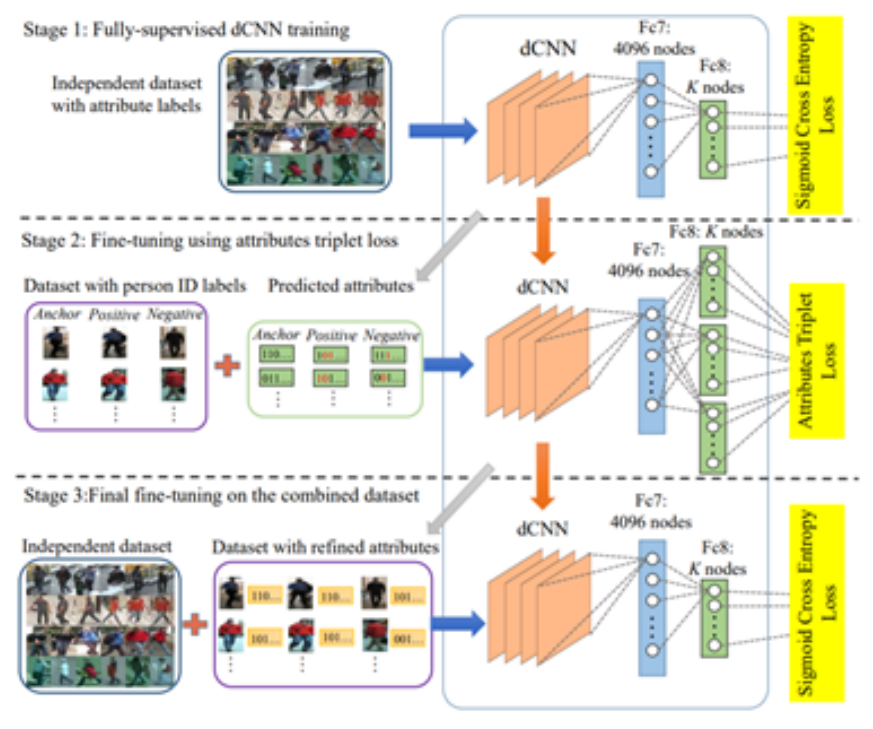

Figure 57. The pipeline of Semi-supervised Deep Attribute Learning (SSDAL). [128].

attributes as real-value variables instead of using them as discrete variables to obtain better person re-ID performance.

\section{Future Research Directions}

In the rest of this section, we discuss several interesting directions for future work of pedestrian attribute recognition. And we also list some released source code of PAR in Table 2.

\subsection{More Accurate and Efficient Part Localization Algo- rithm}

Human beings could recognize the detailed attributes information in an very efficient way, because we can focus on specific regions in a glimpse and reason the attribute based on the local and global information. Therefore, it is an intuitive idea to design algorithms which can detect the local parts for accurate attribute recognition like we humans.

According to section 6.2, it is easy to find that researchers are indeed more interested in mining local parts of human body. They use manual annotated or detected human body or pose information for the part localization. The overall framework of part based attribute recognition can be found in Fig. 58. There are also some algorithms attempt to propose unified framework in a weakly supervised manner to jointly handle the attribute recognition and localization. We think this will also be a good and useful research direction for pedestrian attribute recognition.

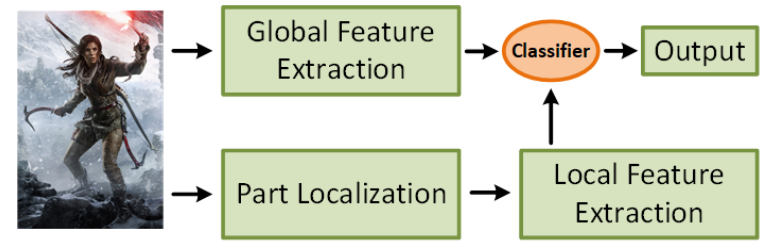

Figure 58. The framework of joint local and global feature extraction for person attribute recognition.

\subsection{Deep Generative Models for Data Augmentation}

In recent years, the deep generative models have achieve great progress and many algorithms are proposed, such as: pixel-CNN [139], pixel-RNN [140], VAE [85], GAN [86]. Recent works like the progressive GAN [141] and bigGAN [142] even make people feel shocked about the image generated by these algorithms. One intuitive research direction is how can we use deep generative models to handle the issues of low-quality person images or unbalanced data distribution?

There are already many researches focus on image generation with the guidance of text, attribute or pose information [143], [144], [145], [146], [147]. The generated images can be used in many other tasks for data augmentation, for example, object detection [148], person re-identification [149], visual tracking [150], et al. GAM [33] also attempt to generate high-resolution images for person attributes recognition. It is also worthy to design new algorithms to generate pedestrian images according to given attributes to augment the training data.

\subsection{Further Explore the Visual Attention Mechanism}

Visual attention has drawn more and more researcher's attention in recent years [151]. It is still one of the most popular techniques used in nowadays and integrated with every kind of deep neural networks in many tasks. Just as noted in [151], one important property of human perception is that one does not tend to process a whole scene in its entirety at once. Instead humans focus attention selectively on parts of the visual space to acquire information when and where it is needed, and combine information from different fixations over time to build up an internal representation of the scene [152], guiding future eye movements and decision making. It also substantially reduces the task complexity as the object of interest can be placed in the center of the fixation and irrelevant features of the visual environment ("clutter") outside the fixated region are naturally ignored.

Many existing attention based pedestrian attribute recognition algorithm focus on feature or task weighting using a trainable neural network. Although it indeed improved the overall recognition performance, however, how to accurately and efficiently locate the attention regions is still an open research problem. Design novel attention mechanism or borrow from other research domains, such as NLP (natural language processing), for pedestrian attribute recognition will be an important research direction in the future.

\subsection{New Designed Loss Functions}

In recent years, there are many loss functions have been proposed for deep neural network optimization, such as (Weighted) Cross Entropy Loss, Contrastive Loss, Center Loss, Triplet Loss, Focal Loss. Researchers also design new loss functions for the PAR, such as WPAL [26], AWMT [27], to further improve their recognition performance. It is an very important direction to study the influence of different loss functions for PAR.

\subsection{Explore More Advanced Network Architecture}

Existing PAR models adopt off the shelf pre-trained network on large scale dataset (such as ImageNet), as their backbone network architecture. Seldom of them consider the unique characteristics of PAR and design novel networks. Some novel networks are proposed in recent years, such as: capsule network [71] [72], External Memory Network [81]. However, there are still no 
attempts to use such networks for PAR. There are also works [153] demonstrate that the deeper network architecture the better recognition performance we can obtain. Nowadays, Automatic Machine Learning solutions (AutoML) draw more and more attentions [154] [155] [156] and many development tools are also released for the development, such as: AutoWEKA [157], Autosklearn [158]. Therefore, it will be a good choice to design specific networks for person attribute recognition in future works with aforementioned approaches.

\subsection{Prior Knowledge guided Learning}

Different from regular classification task, pedestrian attributes recognition always have its own characteristics due to the preference of human beings or natural constraints. It is an important research direction to mining the prior or common knowledge for the PAR. For example, we wear different clothes in various seasons, temperatures or occasions. On the other hand, some researchers attempt to use the history knowledge (such as: Wikipedia ${ }^{\|}$) to help improve their overall performance, such as image caption [159], [160], object detection [161]. Therefore, how to use these information to explore the relations between person attributes or help the machine learning model to further understand the attributes is still an unstudied problems.

\subsection{Multi-modal Pedestrian Attribute Recognition}

Although existing single-modal algorithms already achieve good performance on some benchmark dataset as mentioned above. However, as is known to all, the RGB image is sensitive to illumination, bad weather (such as: rain, snow, fog), night time, et al. It seems impossible for us to achieve accurate pedestrian attributes recognition in all day and all weather. But the actual requirement of intelligent surveillance needs far more than this target. How can we bridge this gap?

One intuitive idea is to mining useful information from other modalities, such as thermal or depth sensors, to integrate with RGB sensor. There are already many works attempt to fuse these multi-modal data and improve their final performance significantly, such as RGB-Thermal tracking [162], [163], [164], moving object detection [165], person re-identification [166], RGB-Depth object detection [167], [168], segmentation [169]. We think the idea of multi-modal fusion could also help improve the robustness of pedestrian attribute recognition. As shown in Fig. 59, these thermal images can highlight the contour of human and some other wearing or carrying objects as denoted in [170], [171].
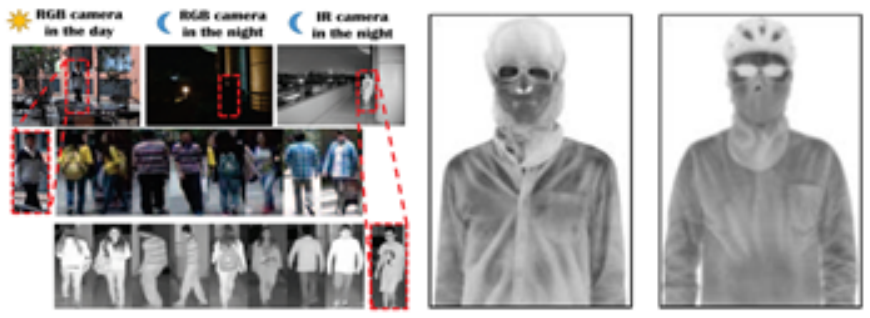

Figure 59. The example RGB and thermal infrared images adopted from [166], [170] .

\| en.wikipedia.org

\subsection{Video based Pedestrian Attribute Recognition}

Existing pedestrian attribute recognition is based on single image, however, in practical scenario we often obtain the video sequence captured by cameras. Although run existing algorithm on each video frame can be an intuitive and easy strategy, but the efficiency maybe the bottleneck for practical applications. Chen et al. propose a video-based PAR dataset [172] by re-annotating MAR dataset [173] which is originally constructed for video based person re-identification. Generally speaking, image based attribute recognition can only make use of the spatial information from the given image, which increased the difficulty of PAR due to the limited information. In contrast, given the video based PAR, we can jointly utilize the spatial and temporal information. The benefits can be listed as follows: 1). we can extend the attribute recognition into a more general case by defining more dynamic person attributes, such as "running man"; 2). the motion information can be used to reasoning the attributes which maybe hard to recognize in single image; 3 ). the general person attributes learned in videos can provide more helpful information for other video based tasks, such as video caption, video object detection. Therefore, how to recognize human attributes in practical video sequence efficiently and accurately is a worthy studying problem.

\subsection{Joint Learning of Attribute and Other Tasks}

Integrate the person attribute learning into the pipeline of other person related tasks is also an interesting and important research direction. There are already many algorithms proposed by consider the person attributes into corresponding tasks, such as: attribute based pedestrian detection [174], visual tracking [175], person reidentification [25], [176], [177], [178] and social activity analysis [179]. In the future works, how to better explore the fine-grained person attributes for other tasks and also use other tasks for better human attribute recognition is an important research direction.

\section{CONCLUSION}

In this paper, we give a review on pedestrian attribute recognition (i.e. PAR) from traditional approaches to deep learning based algorithms in recent years. To the best of our knowledge, this is the first review paper on pedestrian attribute recognition. Specifically speaking, we first introduce the background information (the definition and challenging factors) about PAR. Then, we list existing benchmarks proposed for PAR, including popular datasets and evaluation criteria. After that, we review the algorithms which may used for PAR from two aspects, i.e. the multi-task learning and multi-label learning. Then, we give a brief review of PAR algorithms, we first review some popular neural networks which has widely used in many other tasks; then, we analyse the deep algorithms for PAR from different views, including: global based, part based, visual attention based, sequential prediction based, new designed loss function based, curriculum learning based, graphic model based and other algorithms. Then, we give a short introduction about the previous works on combine person attribute learning and other human related tasks. Finally, we conclude this survey paper and point out some possible research directions from nine aspects for the PAR.

\section{REFERENCES}

[1] N. Dalal and B. Triggs, "Histograms of oriented gradients for human detection," in Computer Vision and Pattern Recognition, 2005. CVPR 2005. IEEE Computer Society Conference on, vol. 1. IEEE, 2005, pp. 886-893. 1, 3, 4, 11 
[2] D. G. Lowe, "Distinctive image features from scale-invariant keypoints," International journal of computer vision, vol. 60, no. 2, pp. 91-110, 2004. 1, 22

[3] C.-C. Chang and C.-J. Lin, "Libsvm: a library for support vector machines," ACM transactions on intelligent systems and technology (TIST), vol. 2, no. 3, p. 27, 2011. 1

[4] J. Lafferty, A. McCallum, and F. C. Pereira, "Conditional random fields: Probabilistic models for segmenting and labeling sequence data," 2001.

[5] P. Sudowe, H. Spitzer, and B. Leibe, "Person attribute recognition with a jointly-trained holistic cnn model," in Proceedings of the IEEE International Conference on Computer Vision Workshops, 2015, pp. 8795. 1, 2, 3, 4, 5, 9, 10, 11

[6] D. Li, X. Chen, and K. Huang, "Multi-attribute learning for pedestrian attribute recognition in surveillance scenarios," in Pattern Recognition (ACPR), 2015 3rd IAPR Asian Conference on. IEEE, 2015, pp. 111115. $1,5,9,10,11,25$

[7] A. H. Abdulnabi, G. Wang, J. Lu, and K. Jia, "Multi-task cnn model for attribute prediction," IEEE Transactions on Multimedia, vol. 17, no. 11, pp. 1949-1959, 2015. 1, 5, 9, 10, 11

[8] L. Bourdev, S. Maji, and J. Malik, "Describing people: A poselet-based approach to attribute classification," in Computer Vision (ICCV), 2011 IEEE International Conference on. IEEE, 2011, pp. 1543-1550. 1, 2, $3,4,5,11,12,13,14$

[9] J. Joo, S. Wang, and S.-C. Zhu, "Human attribute recognition by rich appearance dictionary," in Proceedings of the IEEE International Conference on Computer Vision, 2013, pp. 721-728. 1, 5, 11, 14

[10] N. Zhang, M. Paluri, M. Ranzato, T. Darrell, and L. Bourdev, "Panda: Pose aligned networks for deep attribute modeling," in Proceedings of the IEEE conference on computer vision and pattern recognition, 2014, pp. 1637-1644. 1, 5, 11, 12, 13, 14, 25

[11] J. Zhu, S. Liao, D. Yi, Z. Lei, and S. Z. Li, "Multi-label cnn based pedestrian attribute learning for soft biometrics," in Biometrics (ICB), 2015 International Conference on. IEEE, 2015, pp. 535-540. 1, 5, 11, 12,14

[12] G. Gkioxari, R. Girshick, and J. Malik, "Actions and attributes from wholes and parts," in The IEEE International Conference on Computer Vision (ICCV), December 2015. 1, 11, 12, 13, 14

[13] Y. W. S. L. Luwei Yang, Ligeng Zhu and P. Tan, "Attribute recognition from adaptive parts," in Proceedings of the British Machine Vision Conference $(B M V C)$, E. R. H. Richard C. Wilson and W. A. P. Smith, Eds. BMVA Press, September 2016, pp. 81.1-81.11. [Online]. Available: https://dx.doi.org/10.5244/C.30.81 1, 11, 13, 14

[14] A. Diba, A. Mohammad Pazandeh, H. Pirsiavash, and L. Van Gool, "Deepcamp: Deep convolutional action \& attribute mid-level patterns," in Proceedings of the IEEE Conference on Computer Vision and Pattern Recognition, 2016, pp. 3557-3565. 1, 11, 13, 14, 15

[15] D. Li, X. Chen, Z. Zhang, and K. Huang, "Pose guided deep model for pedestrian attribute recognition in surveillance scenarios," in 2018 IEEE International Conference on Multimedia and Expo (ICME). IEEE, 2018, pp. 1-6. 1, 11, 14, 15

[16] Y. Li, C. Huang, C. C. Loy, and X. Tang, "Human attribute recognition by deep hierarchical contexts," in European Conference on Computer Vision. Springer, 2016, pp. 684-700. 1, 2, 3, 4, 11, 14, 16

[17] P. Liu, X. Liu, J. Yan, and J. Shao, "Localization guided learning for pedestrian attribute recognition," 2018. 1, 11, 14, 16

[18] X. Liu, H. Zhao, M. Tian, L. Sheng, J. Shao, S. Yi, J. Yan, and X. Wang, "Hydraplus-net: Attentive deep features for pedestrian analysis," in Proceedings of the IEEE International Conference on Computer Vision, 2017, pp. 350-359. 1, 2, 3, 4, 14, 16, 25

[19] M. S. Sarfraz, A. Schumann, Y. Wang, and R. Stiefelhagen, "Deep viewsensitive pedestrian attribute inference in an end-to-end model," arXiv preprint arXiv:1707.06089, 2017. 1, 14, 15, 16

[20] N. Sarafianos, X. Xu, and I. A. Kakadiaris, "Deep imbalanced attribute classification using visual attention aggregation," in European Conference on Computer Vision. Springer, 2018, pp. 708-725. 1, 14, 15, 16, 25

[21] H. Guo, X. Fan, and S. Wang, "Human attribute recognition by refining attention heat map," Pattern Recognition Letters, vol. 94, pp. 38-45, 2017. 1, 14, 17

[22] J. Wang, Y. Yang, J. Mao, Z. Huang, C. Huang, and W. Xu, “Cnn-rnn: A unified framework for multi-label image classification," in Proceedings of the IEEE conference on computer vision and pattern recognition, 2016, pp. 2285-2294. 1, 17, 18

[23] J. Wang, X. Zhu, S. Gong, and W. Li, "Attribute recognition by joint recurrent learning of context and correlation," in Computer Vision
(ICCV), 2017 IEEE International Conference on. IEEE, 2017, pp. 531-540. 1, 17, 18

[24] X. Zhao, L. Sang, G. Ding, Y. Guo, and X. Jin, "Grouping attribute recognition for pedestrian with joint recurrent learning." in IJCAI, 2018 , pp. 3177-3183. 1, 17, 18, 19

[25] H. Liu, J. Wu, J. Jiang, M. Qi, and R. Bo, "Sequence-based person attribute recognition with joint ctc-attention model," arXiv preprint arXiv:1811.08115, 2018. 1, 17, 18, 19, 27

[26] Y. Zhou, K. Yu, B. Leng, Z. Zhang, D. Li, K. Huang, B. Feng, C. Yao et al., "Weakly-supervised learning of mid-level features for pedestrian attribute recognition and localization," in $B M V C, 2017.1,19,20,25$, 26

[27] K. He, Z. Wang, Y. Fu, R. Feng, Y.-G. Jiang, and X. Xue, "Adaptively weighted multi-task deep network for person attribute classification," in Proceedings of the 2017 ACM on Multimedia Conference. ACM, 2017, pp. 1636-1644. 1, 19, 20, 26

[28] H. Chen, A. Gallagher, and B. Girod, "Describing clothing by semantic attributes," in Computer Vision - ECCV 2012, A. Fitzgibbon, S. Lazebnik, P. Perona, Y. Sato, and C. Schmid, Eds. Berlin, Heidelberg: Springer Berlin Heidelberg, 2012, pp. 609-623. 1, 4, 22

[29] S. Park, B. X. Nie, and S.-C. Zhu, "Attribute and-or grammar for joint parsing of human pose, parts and attributes," IEEE transactions on pattern analysis and machine intelligence, vol. 40, no. 7, pp. 1555 1569, 2018. 1, 22, 23

[30] Q. L. X. Z. R. H. K. HUANG, "Visual-semantic graph reasoning for pedestrian attribute recognition," in Association for the Advancement of Artificial Intelligence, AAAI, 2019. 1, 22, 23

[31] P. Sudowe and B. Leibe, "Patchit: Self-supervised network weight initialization for fine-grained recognition." in BMVC, 2016. 1, 24, 25

[32] Y. Lu, A. Kumar, S. Zhai, Y. Cheng, T. Javidi, and R. Feris, "Fullyadaptive feature sharing in multi-task networks with applications in person attribute classification," in CVPR, vol. 1, no. 2, 2017, p. 6. 1, 24, 25

[33] M. Fabbri, S. Calderara, and R. Cucchiara, "Generative adversarial models for people attribute recognition in surveillance," in Advanced Video and Signal Based Surveillance (AVSS), 2017 14th IEEE International Conference on. IEEE, 2017, pp. 1-6. 1, 24, 25, 26

[34] Y. Deng, P. Luo, C. C. Loy, and X. Tang, "Pedestrian attribute recognition at far distance," in Proceedings of the 22nd ACM international conference on Multimedia. ACM, 2014, pp. 789-792. 2, 3, 4

[35] D. Li, Z. Zhang, X. Chen, H. Ling, and K. Huang, "A richly annotated dataset for pedestrian attribute recognition," arXiv preprint arXiv:1603.07054, 2016. 2, 3, 4

[36] D. Li, Z. Zhang, X. Chen, and K. Huang, "A richly annotated pedestrian dataset for person retrieval in real surveillance scenarios," IEEE transactions on image processing, vol. 28, no. 4, pp. 1575-1590, 2019. 2, 3 , 4, 25

[37] Y. Lin, L. Zheng, and W. Y. a. Y. Y. Zheng, Zhedong and, "Improving person re-identification by attribute and identity learning," arXiv preprint arXiv:1703.07220, 2017. 2, 4

[38] H. Chen, A. Gallagher, and B. Girod, "Describing clothing by semantic attributes," in European conference on computer vision. Springer, 2012, pp. 609-623. 2, 4

[39] P. Sudowe and B. Leibe, "PatchIt: Self-Supervised Network Weight Initialization for Fine-grained Recognition," in BMVC, 2016. 2, 3, 4

[40] J. Zhu, S. Liao, Z. Lei, D. Yi, and S. Li, "Pedestrian attribute classification in surveillance: Database and evaluation," in Proceedings of the IEEE International Conference on Computer Vision Workshops, 2013, pp. 331-338. 2, 3, 4

[41] G. Sharma and F. Jurie, "Learning discriminative spatial representation for image classification," in BMVC 2011-British Machine Vision Conference. BMVA Press, 2011, pp. 1-11. 2, 3, 4

[42] D. Hall and P. Perona, "Fine-grained classification of pedestrians in video: Benchmark and state of the art," in Proceedings of the IEEE Conference on Computer Vision and Pattern Recognition, 2015, pp. 5482-5491. 3, 4

[43] Y. Xiong, K. Zhu, D. Lin, and X. Tang, "Recognize complex events from static images by fusing deep channels," in 2015 IEEE Conference on Computer Vision and Pattern Recognition (CVPR), June 2015, pp. 1600-1609. 3, 4

[44] A. Geiger, P. Lenz, and R. Urtasun, "Are we ready for autonomous driving? the kitti vision benchmark suite," in Computer Vision and Pattern Recognition (CVPR), 2012 IEEE Conference on. IEEE, 2012, pp. 3354-3361. 3, 4

[45] S. M. Bileschi, "Streetscenes: Towards scene understanding in still images," MASSACHUSETTS INST OF TECH CAMBRIDGE, Tech. Rep., 2006. 3, 4 
[46] L. Bourdev and J. Malik, "Poselets: Body part detectors trained using 3d human pose annotations," in Computer Vision, 2009 IEEE 12th International Conference on. IEEE, 2009, pp. 1365-1372. 3, 4, 11

[47] M. Everingham, L. Van Gool, C. K. I. Williams, J. Winn, and A. Zisserman, "The PASCAL Visual Object Classes Challenge 2010 (VOC2010) Results," http://www.pascalnetwork.org/challenges/VOC/voc2010/workshop/index.html. 4

[48] P. F. Felzenszwalb, R. B. Girshick, D. McAllester, and D. Ramanan, "Object detection with discriminatively trained part-based models," IEEE transactions on pattern analysis and machine intelligence, vol. 32, no. 9, pp. 1627-1645, 2010. 3

[49] J. Yan, Z. Lei, D. Yi, and S. Z. Li, "Multi-pedestrian detection in crowded scenes: A global view," in Computer Vision and Pattern Recognition (CVPR), 2012 IEEE Conference on. IEEE, 2012, pp. 3124-3129. 3

[50] Y. Lin, L. Zheng, Z. Zheng, Y. Wu, and Y. Yang, "Improving person re-identification by attribute and identity learning," CoRR, vol. abs/1703.07220, 2017. [Online]. Available: http://arxiv.org/abs/1703. 072203

[51] M.-L. Zhang and Z.-H. Zhou, "A review on multi-label learning algorithms," IEEE transactions on knowledge and data engineering, vol. 26, no. 8, pp. 1819-1837, 2014. 4, 5

[52] S. Ruder, "An overview of multi-task learning in deep neural networks," arXiv preprint arXiv:1706.05098, 2017. 5

[53] L. Duong, T. Cohn, S. Bird, and P. Cook, "Low resource dependency parsing: Cross-lingual parameter sharing in a neural network parser," in Proceedings of the 53rd Annual Meeting of the Association for Computational Linguistics and the 7th International Joint Conference on Natural Language Processing (Volume 2: Short Papers), vol. 2, 2015, pp. 845-850. 5

[54] Y. Yang and T. M. Hospedales, "Trace norm regularised deep multi-task learning," arXiv preprint arXiv:1606.04038, 2016. 5

[55] M. R. Boutell, J. Luo, X. Shen, and C. M. Brown, "Learning multi-labe scene classification," Pattern recognition, vol. 37, no. 9, pp. 1757-1771, 2004. 5

[56] J. Read, B. Pfahringer, G. Holmes, and E. Frank, "Classifier chains for multi-label classification," Machine learning, vol. 85, no. 3, p. 333, 2011. 5

[57] J. Fürnkranz, E. Hüllermeier, E. L. Mencía, and K. Brinker, "Multilabel classification via calibrated label ranking," Machine learning, vol. 73, no. 2 , pp. $133-153,2008.5$

[58] G. Tsoumakas, I. Katakis, and I. Vlahavas, "Random k-labelsets for multilabel classification," IEEE Transactions on Knowledge and Data Engineering, vol. 23, no. 7, pp. 1079-1089, 2011. 5

[59] M.-L. Zhang and Z.-H. Zhou, "Ml-knn: A lazy learning approach to multi-label learning," Pattern recognition, vol. 40, no. 7, pp. 2038-2048, 2007. 6

[60] A. Clare and R. D. King, "Knowledge discovery in multi-label phenotype data," in European Conference on Principles of Data Mining and Knowledge Discovery. Springer, 2001, pp. 42-53. 6

[61] A. Elisseeff and J. Weston, "A kernel method for multi-labelled classification," in Advances in neural information processing systems, 2002, pp. $681-687.6$

[62] N. Ghamrawi and A. McCallum, "Collective multi-label classification," in Proceedings of the 14th ACM international conference on Information and knowledge management. ACM, 2005, pp. 195-200. 6

[63] Y. LeCun, L. Bottou, Y. Bengio, and P. Haffner, "Gradient-based learning applied to document recognition," Proceedings of the IEEE, vol. 86 , no. 11 , pp. $2278-2324,1998.6$

[64] A. Krizhevsky, I. Sutskever, and G. E. Hinton, "Imagenet classification with deep convolutional neural networks," in Advances in neural information processing systems, 2012, pp. 1097-1105. 6, 14

[65] K. Simonyan and A. Zisserman, "Very deep convolutional networks for large-scale image recognition," arXiv preprint arXiv:1409.1556, 2014. 6

[66] K. He, X. Zhang, S. Ren, and J. Sun, "Deep residual learning for image recognition," in Proceedings of the IEEE conference on computer vision and pattern recognition, 2016, pp. 770-778. 6, 7,8

[67] C. Szegedy, W. Liu, Y. Jia, P. Sermanet, S. Reed, D. Anguelov, D. Erhan, V. Vanhoucke, A. Rabinovich et al., "Going deeper with convolutions." Cvpr, 2015. 6, 8

[68] M. Lin, Q. Chen, and S. Yan, "Network in network," arXiv preprint arXiv:1312.4400, 2013. 6

[69] G. Huang, Z. Liu, K. Q. Weinberger, and L. van der Maaten, "Densely connected convolutional networks," in Proceedings of the IEEE confer- ence on computer vision and pattern recognition, vol. 1, no. 2, 2017, p. $3.6,8$

[70] C.-Y. Lee, S. Xie, P. Gallagher, Z. Zhang, and Z. Tu, "Deeply-supervised nets," in Artificial Intelligence and Statistics, 2015, pp. 562-570. 6

[71] S. Sabour, N. Frosst, and G. E. Hinton, "Dynamic routing between capsules," in Advances in Neural Information Processing Systems, 2017, pp. 3859-3869. 6, 8, 26

[72] G. Hinton, N. Frosst, and S. Sabour, "Matrix capsules with em routing," 2018. 6, 26

[73] T. N. Kipf and M. Welling, "Semi-supervised classification with graph convolutional networks," arXiv preprint arXiv:1609.02907, 2016. 6

[74] F. Visin, K. Kastner, K. Cho, M. Matteucci, A. Courville, and Y. Bengio, "Renet: A recurrent neural network based alternative to convolutional networks," arXiv preprint arXiv:1505.00393, 2015. 6, 8

[75] A. Graves and J. Schmidhuber, "Offline handwriting recognition with multidimensional recurrent neural networks," in Advances in neural information processing systems, 2009, pp. 545-552. 6

[76] K. Cho, B. Van Merriënboer, C. Gulcehre, D. Bahdanau, F. Bougares, H. Schwenk, and Y. Bengio, "Learning phrase representations using rnn encoder-decoder for statistical machine translation," arXiv preprint arXiv:1406.1078, 2014. 8

[77] O. Irsoy and C. Cardie, "Deep recursive neural networks for compositionality in language," in Advances in neural information processing systems, 2014, pp. 2096-2104. 8

[78] J. Gehring, M. Auli, D. Grangier, D. Yarats, and Y. N. Dauphin, "Convolutional sequence to sequence learning," in International Conference on Machine Learning, 2017, pp. 1243-1252. 9, 23

[79] R. Johnson and T. Zhang, "Effective use of word order for text categorization with convolutional neural networks," in Proceedings of the 2015 Conference of the North American Chapter of the Association for Computational Linguistics: Human Language Technologies, 2015, pp. 103-112. 9

[80] X. Wang, C. Li, R. Yang, T. Zhang, J. Tang, and B. Luo, "Describe and attend to track: Learning natural language guided structural representation and visual attention for object tracking," arXiv preprint arXiv:1811.10014, 2018. 9

[81] A. Graves, G. Wayne, M. Reynolds, T. Harley, I. Danihelka, A. GrabskaBarwińska, S. G. Colmenarejo, E. Grefenstette, T. Ramalho, J. Agapiou et al., "Hybrid computing using a neural network with dynamic external memory," Nature, vol. 538, no. 7626, p. 471, 2016. 9, 26

[82] T. Yang and A. B. Chan, "Learning dynamic memory networks for object tracking," in The European Conference on Computer Vision (ECCV), September 2018. 9

[83] C. Xiong, S. Merity, and R. Socher, "Dynamic memory networks for visual and textual question answering," in International conference on machine learning, 2016, pp. 2397-2406. 9

[84] C. Ma, C. Shen, A. Dick, Q. Wu, P. Wang, A. van den Hengel, and I. Reid, "Visual question answering with memory-augmented networks," in The IEEE Conference on Computer Vision and Pattern Recognition (CVPR), June 2018. 9

[85] C. Doersch, "Tutorial on variational autoencoders," arXiv preprint arXiv:1606.05908, 2016. 9, 26

[86] I. Goodfellow, J. Pouget-Abadie, M. Mirza, B. Xu, D. Warde-Farley, S. Ozair, A. Courville, and Y. Bengio, "Generative adversarial nets", in Advances in neural information processing systems, 2014, pp. 2672 2680. 9,26

[87] M. Mirza and S. Osindero, "Conditional generative adversarial networks," Manuscript: https://arxiv. org/abs/1709.02023, 2014. 9, 24

[88] L. Bourdev, F. Yang, and R. Fergus, "Deep poselets for human detection," arXiv preprint arXiv: 1407.0717, 2014. 11

[89] N. Jammalamadaka, A. Zisserman, and C. Jawahar, "Human pose search using deep poselets," in Automatic Face and Gesture Recognition $(F G), 2015$ 11th IEEE International Conference and Workshops on, vol. 1. IEEE, 2015, pp. 1-8. 11

[90] S. Lazebnik, C. Schmid, and J. Ponce, "Beyond bags of features: Spatia pyramid matching for recognizing natural scene categories," in null. IEEE, 2006, pp. 2169-2178. 11

[91] L. Bourdev, S. Maji, T. Brox, and J. Malik, "Detecting people using mutually consistent poselet activations," in European conference on computer vision. Springer, 2010, pp. 168-181. 13

[92] R. Girshick, F. Iandola, T. Darrell, and J. Malik, "Deformable part models are convolutional neural networks," in Proceedings of the IEEE conference on Computer Vision and Pattern Recognition, 2015, pp. 437446. 13

[93] Y. Li, L. Liu, C. Shen, and A. van den Hengel, "Mid-level deep pattern mining," in Proceedings of the IEEE Conference on Computer Vision and Pattern Recognition, 2015, pp. 971-980. 13 
[94] D. Li, X. Chen, and K. Huang, "Multi-attribute learning for pedestrian attribute recognition in surveillance scenarios," in Pattern Recognition (ACPR), 2015 3rd IAPR Asian Conference on. IEEE, 2015, pp. 111115. 14

[95] M. Jaderberg, K. Simonyan, A. Zisserman et al., "Spatial transformer networks," in Advances in neural information processing systems, 2015, pp. 2017-2025. 14

[96] S. Ioffe and C. Szegedy, "Batch normalization: Accelerating deep network training by reducing internal covariate shift," in International Conference on Machine Learning, 2015, pp. 448-456. 14

[97] C. L. Zitnick and P. Dollár, "Edge boxes: Locating object proposals from edges," in European conference on computer vision. Springer, 2014, pp. 391-405. 14

[98] S. Ioffe and C. Szegedy, "Batch normalization: Accelerating deep network training by reducing internal covariate shift," in International Conference on Machine Learning, 2015, pp. 448-456. 15

[99] T.-Y. Lin, P. Goyal, R. Girshick, K. He, and P. Dollár, "Focal loss for dense object detection," IEEE transactions on pattern analysis and machine intelligence, 2018. 15

[100] H.-S. Chang, E. Learned-Miller, and A. McCallum, "Active bias: Training more accurate neural networks by emphasizing high variance samples," in Advances in Neural Information Processing Systems, 2017, pp. 1002-1012. 16

[101] B. Zhou, A. Lapedriza, J. Xiao, A. Torralba, and A. Oliva, "Learning deep features for scene recognition using places database," in Advances in neural information processing systems, 2014, pp. 487-495. 17

[102] G. D. J. H. N. D. Xin Zhao, Liufang Sang and C. Yan, "Recurrent attention model for pedestrian attribute recognition ," in Association for the Advancement of Artificial Intelligence, AAAI, 2019. 17, 19

[103] D. R. Reddy et al., "Speech understanding systems: A summary of results of the five-year research effort," Department of Computer Science. Camegie-Mell University, Pittsburgh, PA, 1977. 17

[104] O. Vinyals, A. Toshev, S. Bengio, and D. Erhan, "Show and tell: A neural image caption generator," in Proceedings of the IEEE conference on computer vision and pattern recognition, 2015, pp. 3156-3164. 17

[105] A. Mathews, L. Xie, and X. He, "Semstyle: Learning to generate stylised image captions using unaligned text," in The IEEE Conference on Computer Vision and Pattern Recognition (CVPR), June 2018. 17

[106] A. Vaswani, N. Shazeer, N. Parmar, J. Uszkoreit, L. Jones, A. N. Gomez, Ł. Kaiser, and I. Polosukhin, "Attention is all you need," in Advances in Neural Information Processing Systems, 2017, pp. 5998-6008. 18

[107] A. Graves, S. Fernández, F. Gomez, and J. Schmidhuber, "Connectionist temporal classification: labelling unsegmented sequence data with recurrent neural networks," in Proceedings of the 23rd international conference on Machine learning. ACM, 2006, pp. 369-376. 18

[108] S. Xingjian, Z. Chen, H. Wang, D.-Y. Yeung, W.-K. Wong, and W.c. Woo, "Convolutional lstm network: A machine learning approach for precipitation nowcasting," in Advances in neural information processing systems, 2015, pp. 802-810. 19

[109] Q. Dong, S. Gong, and X. Zhu, "Multi-task curriculum transfer deep learning of clothing attributes," in Applications of Computer Vision (WACV), 2017 IEEE Winter Conference on. IEEE, 2017, pp. 520 529. 20, 21

[110] N. Sarafianos, T. Giannakopoulos, C. Nikou, and I. A. Kakadiaris, "Curriculum learning for multi-task classification of visual attributes," in Proceedings of the IEEE International Conference on Computer Vision, 2017, pp. 2608-2615. 20, 21

[111] M. Lin, Q. Chen, and S. Yan, "Network in network," arXiv preprint arXiv:1312.4400, 2013. 21

[112] N. Sarafianos, T. Giannakopoulos, C. Nikou, and I. A. Kakadiaris, "Curriculum learning of visual attribute clusters for multi-task classification," Pattern Recognition, vol. 80, pp. 94-108, 2018. 21

[113] J. Zhu, S. Liao, Z. Lei, and S. Z. Li, "Multi-label convolutional neural network based pedestrian attribute classification," Image and Vision Computing, vol. 58, pp. 224-229, 2017. 21

[114] B. Zhang, L. Wang, Z. Wang, Y. Qiao, and H. Wang, "Real-time action recognition with enhanced motion vector cnns," in Proceedings of the IEEE Conference on Computer Vision and Pattern Recognition, 2016, pp. 2718-2726. 21

[115] M. P. Kumar, B. Packer, and D. Koller, "Self-paced learning for latent variable models," in Advances in Neural Information Processing Systems, 2010, pp. 1189-1197. 22

[116] C. Li, F. Wei, J. Yan, X. Zhang, Q. Liu, and H. Zha, "A self-paced regularization framework for multilabel learning," IEEE transactions on neural networks and learning systems, vol. 29, no. 6, pp. 2660-2666, 2018. 22
[117] D. Zhang, D. Meng, and J. Han, "Co-saliency detection via a self-paced multiple-instance learning framework," IEEE transactions on pattern analysis and machine intelligence, vol. 39, no. 5, pp. 865-878, 2017. 22

[118] M. Eichner, M. Marin-Jimenez, A. Zisserman, and V. Ferrari, "Articulated human pose estimation and search in (almost) unconstrained still images," ETH Zurich, D-ITET, BIWI, Technical Report No, vol. 272, 2010. 22

[119] M. Varma and A. Zisserman, "A statistical approach to texture classification from single images," International journal of computer vision, vol. 62, no. 1-2, pp. 61-81, 2005. 22

[120] M. F. Tappen and W. T. Freeman, "Comparison of graph cuts with belief propagation for stereo, using identical mrf parameters," in null. IEEE, 2003, p. 900.22

[121] S.-C. Zhu, D. Mumford et al., "A stochastic grammar of images,"

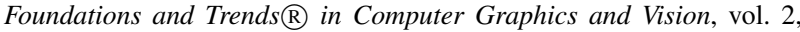
no. 4, pp. 259-362, 2007. 22, 24

[122] S. Park and S.-C. Zhu, "Attributed grammars for joint estimation of human attributes, part and pose," in Proceedings of the IEEE International Conference on Computer Vision, 2015, pp. 2372-2380. 23

[123] S. Z. Li, "Markov random field models in computer vision," in European conference on computer vision. Springer, 1994, pp. 361-370. 24

[124] J. Lafferty, A. McCallum, and F. C. Pereira, "Conditional random fields: Probabilistic models for segmenting and labeling sequence data," 2001. 24

[125] M. Henaff, J. Bruna, and Y. LeCun, "Deep convolutional networks on graph-structured data," arXiv preprint arXiv:1506.05163, 2015. 24

[126] J. A. Tropp, A. C. Gilbert, and M. J. Strauss, "Algorithms for simultaneous sparse approximation. part i: Greedy pursuit," Signal processing, vol. 86 , no. 3, pp. 572-588, 2006. 24

[127] Z. Wang, T. Chen, G. Li, R. Xu, and L. Lin, "Multi-label image recognition by recurrently discovering attentional regions," in Proceedings of the IEEE Conference on Computer Vision and Pattern Recognition, 2017, pp. 464-472. 25

[128] C. Su, S. Zhang, J. Xing, W. Gao, and Q. Tian, "Deep attributes driven multi-camera person re-identification," in European conference on computer vision. Springer, 2016, pp. 475-491. 24, 25

[129] R. Layne, T. M. Hospedales, and S. Gong, "Towards person identification and re-identification with attributes," in European Conference on Computer Vision. Springer, 2012, pp. 402-412. 24

[130] S. Khamis, C.-H. Kuo, V. K. Singh, V. D. Shet, and L. S. Davis, "Join learning for attribute-consistent person re-identification," in European Conference on Computer Vision. Springer, 2014, pp. 134-146. 24, 26

[131] A. Li, L. Liu, K. Wang, S. Liu, and S. Yan, "Clothing attributes assisted person reidentification," IEEE Transactions on Circuits and Systems for Video Technology, vol. 25, no. 5, pp. 869-878, 2015. 24, 26

[132] Y. Lin, L. Zheng, Z. Zheng, Y. Wu, and Y. Yang, "Improving person re-identification by attribute and identity learning," arXiv preprint arXiv:1703.07220, 2017. 24, 25

[133] Y. Tian, P. Luo, X. Wang, and X. Tang, "Pedestrian detection aided by deep learning semantic tasks," in Proceedings of the IEEE Conference on Computer Vision and Pattern Recognition, 2015, pp. 5079-5087. 24, 25

[134] X. Liu, Y. Xu, L. Zhu, and Y. Mu, "A stochastic attribute grammar for robust cross-view human tracking," IEEE transactions on circuits and systems for video technology, 2018. 24

[135] X. Wang, T. Zhang, D. R. Tretter, and Q. Lin, "Personal clothing retrieval on photo collections by color and attributes," IEEE Transactions on Multimedia, vol. 15, no. 8, pp. 2035-2045, 2013. 24

[136] R. Feris, R. Bobbitt, L. Brown, and S. Pankanti, "Attribute-based people search: Lessons learnt from a practical surveillance system," in Proceedings of International Conference on Multimedia Retrieval. ACM, 2014, p. 153. 24

[137] J. Liu, B. Kuipers, and S. Savarese, "Recognizing human actions by attributes," in Computer Vision and Pattern Recognition (CVPR), 2011 IEEE Conference on. IEEE, 2011, pp. 3337-3344. 24

[138] J. Shao, K. Kang, C. Change Loy, and X. Wang, "Deeply learned attributes for crowded scene understanding," in Proceedings of the IEEE conference on computer vision and pattern recognition, 2015, pp. 46574666. 24

[139] A. van den Oord, N. Kalchbrenner, L. Espeholt, O. Vinyals, A. Graves et al., "Conditional image generation with pixelcnn decoders," in Advances in Neural Information Processing Systems, 2016, pp. 4790 4798. 26

[140] A. Van Oord, N. Kalchbrenner, and K. Kavukcuoglu, "Pixel recurrent neural networks," in International Conference on Machine Learning, 2016, pp. 1747-1756. 26 
[141] T. Karras, T. Aila, S. Laine, and J. Lehtinen, "Progressive growing of gans for improved quality, stability, and variation," arXiv preprint arXiv:1710.10196, 2017. 26

[142] A. Brock, J. Donahue, and K. Simonyan, "Large scale gan training for high fidelity natural image synthesis," arXiv preprint arXiv:1809.11096, 2018. 26

[143] L. Ma, X. Jia, Q. Sun, B. Schiele, T. Tuytelaars, and L. Van Gool, "Pose guided person image generation," in Advances in Neural Information Processing Systems, 2017, pp. 406-416. 26

[144] X. Qian, Y. Fu, T. Xiang, W. Wang, J. Qiu, Y. Wu, Y.-G. Jiang, and $\mathrm{X}$. Xue, "Pose-normalized image generation for person reidentification," in Proceedings of the European Conference on Computer Vision (ECCV), 2018, pp. 650-667. 26

[145] X. Yan, J. Yang, K. Sohn, and H. Lee, "Attribute2image: Conditional image generation from visual attributes," in European Conference on Computer Vision. Springer, 2016, pp. 776-791. 26

[146] A. Fu and Y. Hou, "Text-to-image generation using multi-instance stackgan," Department of Computer Science Stanford University Stanford, CA, vol. 94305, 2016. 26

[147] H. Zhang, T. Xu, H. Li, S. Zhang, X. Wang, X. Huang, and D. N. Metaxas, "Stackgan: Text to photo-realistic image synthesis with stacked generative adversarial networks," in Proceedings of the IEEE International Conference on Computer Vision, 2017, pp. 5907-5915. 26

[148] X. Wang, A. Shrivastava, and A. Gupta, "A-fast-renn: Hard positive generation via adversary for object detection," in IEEE Conference on Computer Vision and Pattern Recognition, 2017. 26

[149] Z. Zheng, L. Zheng, and Y. Yang, "Unlabeled samples generated by gan improve the person re-identification baseline in vitro," in Proceedings of the IEEE International Conference on Computer Vision, 2017, pp. 3754-3762. 26

[150] X. Wang, C. Li, B. Luo, and J. Tang, "Sint++: Robust visual tracking via adversarial positive instance generation," in Proceedings of the IEEE Conference on Computer Vision and Pattern Recognition, 2018, pp. 4864-4873. 26

[151] V. Mnih, N. Heess, A. Graves et al., "Recurrent models of visual attention," in Advances in neural information processing systems, 2014, pp. 2204-2212. 26

[152] R. A. Rensink, "The dynamic representation of scenes," Visual cognition, vol. 7, no. 1-3, pp. 17-42, 2000. 26

[153] E. Bekele and W. Lawson, "The deeper, the better: Analysis of person attributes recognition," arXiv preprint arXiv:1901.03756, 2019. 26

[154] F. S. H. A. W. H. A. Y. L. F.-F. Chenxi Liu, Liang-Chieh Chen, "Autodeeplab: Hierarchical neural architecture search for semantic image segmentation," arXiv preprint arXiv:1901.02985, 2019. 26

[155] T. Elsken, J. H. Metzen, and F. Hutter, "Neural architecture search: A survey," arXiv preprint arXiv:1808.05377, 2018. 26

[156] Y. He, J. Lin, Z. Liu, H. Wang, L.-J. Li, and S. Han, "Amc: Automl for model compression and acceleration on mobile devices," in European Conference on Computer Vision. Springer, 2018, pp. 815-832. 26

[157] C. Thornton, F. Hutter, H. H. Hoos, and K. Leyton-Brown, "AutoWEKA: Combined selection and hyperparameter optimization of classification algorithms," in Proc. of KDD-2013, 2013, pp. 847-855. 27

[158] M. Feurer, A. Klein, K. Eggensperger, J. Springenberg, M. Blum, and F. Hutter, "Efficient and robust automated machine learning," in Advances in Neural Information Processing Systems 28, C. Cortes, N. D. Lawrence, D. D. Lee, M. Sugiyama, and R. Garnett, Eds. Curran Associates, Inc., 2015, pp. 2962-2970. [Online]. Available: http://papers.nips.cc/paper/ 5872-efficient-and-robust-automated-machine-learning.pdf 27

[159] Q. Wu, C. Shen, P. Wang, A. Dick, and d. H. A. Van, "Image captioning and visual question answering based on attributes and external knowledge." IEEE Transactions on Pattern Analysis and Machine Intelligence, vol. PP, no. 99, pp. 1-1, 2017. 27

[160] W. Peng, W. Qi, C. Shen, A. V. D. Hengel, and A. Dick, "Explicit knowledge-based reasoning for visual question answering," Computer Science, 2015. 27

[161] X. L. L. L. ChenHan Jiang, Hang Xu, "Hybrid knowledge routed modules for large-scale object detection," in NIPS, 2018. 27

[162] C. Li, X. Sun, X. Wang, L. Zhang, and J. Tang, "Grayscale-thermal object tracking via multitask laplacian sparse representation," IEEE Transactions on Systems, Man, and Cybernetics: Systems, vol. 47, no. 4, pp. 673-681, 2017. 27

[163] C. Li, H. Cheng, S. Hu, X. Liu, J. Tang, and L. Lin, "Learning collaborative sparse representation for grayscale-thermal tracking," IEEE Transactions on Image Processing, vol. 25, no. 12, pp. 5743-5756, 2016. 27
[164] C. Li, X. Liang, Y. Lu, N. Zhao, and J. Tang, "Rgb-t object tracking: Benchmark and baseline," arXiv preprint arXiv:1805.08982, 2018. 27

[165] C. Li, X. Wang, L. Zhang, J. Tang, H. Wu, and L. Lin, "Weighted lowrank decomposition for robust grayscale-thermal foreground detection," IEEE Transactions on Circuits and Systems for Video Technology, vol. 27 , no. 4 , pp. $725-738,2017.27$

[166] A. Wu, W.-S. Zheng, H.-X. Yu, S. Gong, and J. Lai, "Rgb-infrared cross-modality person re-identification," in 2017 IEEE International Conference on Computer Vision (ICCV). IEEE, 2017, pp. 5390-5399. 27

[167] S. Gupta, R. Girshick, P. Arbeláez, and J. Malik, "Learning rich features from rgb-d images for object detection and segmentation," in European Conference on Computer Vision. Springer, 2014, pp. 345-360. 27

[168] S. Song and J. Xiao, "Deep sliding shapes for amodal 3d object detection in rgb-d images," in Proceedings of the IEEE Conference on Computer Vision and Pattern Recognition, 2016, pp. 808-816. 27

[169] N. Silberman, D. Hoiem, P. Kohli, and R. Fergus, "Indoor segmentation and support inference from rgbd images," in European Conference on Computer Vision. Springer, 2012, pp. 746-760. 27

[170] B. Kresnaraman, Y. Kawanishi, T. Takahashi, D. Deguchi, Y. Mekada, I. Ide, and H. Murase, "Human wearable attribute recognition using decomposition of thermal infrared images," in Proc. 22nd Korea-Japan Joint Workshop on Frontiers of Computer Vision, 2016, pp. 123-127. 27

[171] B. Kresnaraman, Y. Kawanishi, D. Deguchi, T. Takahashi, Y. Mekada, I. Ide, and H. Murase, "Headgear recognition by decomposing human images in the thermal infrared spectrum," in Quality in Research (QiR): International Symposium on Electrical and Computer Engineering, 2017 15th International Conference on. IEEE, 2017, pp. 164-168. 27

[172] A. L. Zhiyuan Chen and Y. Wang, "Video-based pedestrian attribute recognition," arXiv preprint arXiv:1901.05742, 2019. 27

[173] L. Zheng, Z. Bie, Y. Sun, J. Wang, C. Su, S. Wang, and Q. Tian, "Mars: A video benchmark for large-scale person re-identification," in European Conference on Computer Vision. S Springer, 2016, pp. 868884. 27

[174] Y. Tian, P. Luo, X. Wang, and X. Tang, "Pedestrian detection aided by deep learning semantic tasks," in Proceedings of the IEEE Conference on Computer Vision and Pattern Recognition, 2015, pp. 5079-5087. 27

[175] M. Danelljan, F. Shahbaz Khan, M. Felsberg, and J. Van de Weijer, "Adaptive color attributes for real-time visual tracking," in Proceedings of the IEEE Conference on Computer Vision and Pattern Recognition, 2014, pp. 1090-1097. 27

[176] C. Su, S. Zhang, J. Xing, W. Gao, and Q. Tian, "Deep attributes driven multi-camera person re-identification," in European conference on computer vision. Springer, 2016, pp. 475-491. 27

[177] R. Layne, T. M. Hospedales, and S. Gong, "Towards person identification and re-identification with attributes," in European Conference on Computer Vision. Springer, 2012, pp. 402-412. 27

[178] A. Li, L. Liu, K. Wang, S. Liu, and S. Yan, "Clothing attributes assisted person reidentification," IEEE Transactions on Circuits and Systems for Video Technology, vol. 25, no. 5, pp. 869-878, 2015. 27

[179] Y. Fu, T. M. Hospedales, T. Xiang, and S. Gong, "Attribute learning for understanding unstructured social activity," in European Conference on Computer Vision. $\quad$ Springer, 2012, pp. 530-543. 27

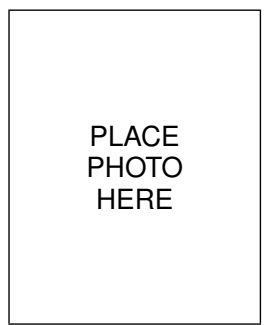

Xiao Wang received the B.S. degree in Western Anhui University, Luan, China, in 2013. He is currently pursuing the Ph.D. degree in computer science in Anhui University. From 2015 and 2016, he was a visiting student with the School of Data and Computer Science, Sun Yatsen University, Guangzhou, China. His current research interests mainly about computer vision, machine learning, pattern recognition and deep learning. 


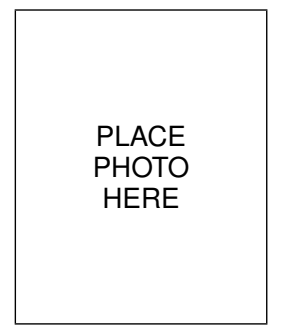

Shaofei Zheng received the B.S. degree in Anhui Polytechnic University, Wuhu, China, in 2015. He is currently pursuing the Master degree in computer science in Anhui University. His current research interests mainly about computer vision and machine learning.

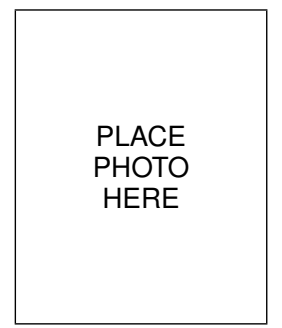

Rui Yang received the B.S. degree in Anhui University of Technology, Ma'anshan, China, in 2018. She is currently pursuing the Master degree in computer science in Anhui University. Her current research interests mainly about computer vision and machine learning.

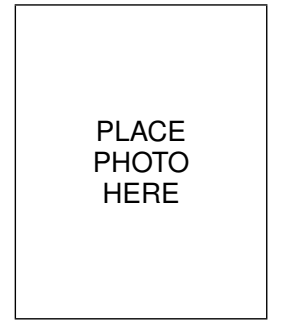

Bin Luo received the B.Eng. degree in electronics and the M.Eng. degree in computer science from Anhui University, Hefei, China, in 1984 and 1991, respectively, and the Ph.D. degree in computer science from the University of York, York, U.K., in 2002. He has authored over 200 papers in journals, edited books, and refereed conferences. He is currently a Professor with Anhui University. He also chairs the IEEE Hefei Subsection. He served as a peer reviewer of international academic journals, such as the IEEE TRANSACTIONS ON PATTERN ANALYSIS AND MACHINE INTELLIGENCE, Pattern Recognition, Pattern Recognition Letters, the International Journal of Pattern Recognition and Artificial Intelligence, Knowledge and Information Systems, and Neurocomputing. His current research interests include random graph-based pattern recognition, image and graph matching, graph spectral analysis, and video analysis.

\begin{tabular}{|l|}
\hline \\
PLACE \\
PHOTO \\
HERE \\
\end{tabular}

Jin Tang received the B.Eng. Degree in automation and the Ph.D. degree in computer science from Anhui University, Hefei, China, in 1999 and 2007, respectively. He is currently a Professor with the School of Computer Science and Technology, Anhui University. His current research interests include computer vision, pattern recognition, and machine learning. 